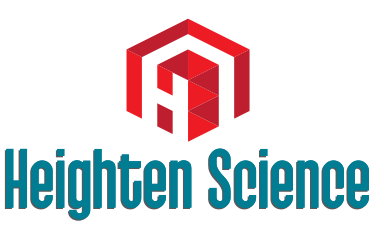

P U B L I C I T I O N S Corporation

\title{
The properties of nonlinear excitations and verification of validity of theory of energy transport in the protein molecules
}

\author{
Pang Xiao-Feng* \\ Institute of Life Science and Technology, University of Electronic Science and Technology of \\ China. Chengdu 610054, China
}

\begin{abstract}
*Address for Correspondence: Pang XiaoFeng, Institute of Life Science and Technology, University of Electronic Science and Technology of China. Chengdu 610054, China. Email: pangxf2006@yahoo.com.cn
\end{abstract}

Submitted: 27 March 2018

Approved: 06 April 2018

Published: 09 April 2018

Copyright: @ 2018 Xiao-Feng P. This is an open access article distributed under the Creative Commons Attribution License, which permits unrestricted use, distribution, and reproduction in any medium, provided the original work is properly cited.

Keywords: Nonlinear excitation; Protein; Energy transport; Soliton; Stability verification; Validity; Numerical simulation; Binding energy; Dynamic equation

\section{Abstract}

Based on different properties of structure of helical protein molecules some theories of bio-energy transport along the molecular chains have been proposed and established, where the energy is released by hydrolysis of adenosine triphosphate (ATP). A brief survey of past researches on different models and theories of bio-energy, including Davydov's, Brown et al's, Schweitzer's, Cruzeiro-Hansson's, Forner's and Pang's models were first stated in this paper. Subsequently we studied and reviewed mainly and systematically the properties and stability of the carriers (solitons) transporting the bio-energy at physiological temperature $300 \mathrm{~K}$ in Pang's and Davydov's theories. However, these theoretical models including Davydov's and Pang's model were all established based on a periodic and uniform proteins, which are different from practically biological proteins molecules. Therefore, it is very necessary to inspect and verify the validity of the theory of bio-energy transport in really biological protein molecules. These problems were extensively studied by a lot of researchers and using different methods in past thirty years, a considerable number of research results were obtained. I here reviewed the situations and progresses of study on this problem, in which we reviewed the correctness of the theory of bio-energy transport including Davydov's and Pang's model and its investigated progresses under influences of structure nonuniformity and disorder, side groups and imported impurities of protein chains as well as the thermal perturbation and damping of medium arising from the biological temperature of the systems. The structure nonuniformity arises from the disorder distribution of sequence of masses of amino acid residues and side groups and imported impurities, which results in the changes and fluctuations of the spring constant, dipole-dipole interaction, exciton-phonon coupling constant, diagonal disorder or ground state energy and chain-chain interaction among the molecular channels in the dynamic equations in different models. The influences of structure nonuniformity, side groups and imported impurities as well as the thermal perturbation and damping of medium on the bio-energy transport in the proteins with single chain and three chains were studied by differently numerical simulation technique and methods containing the average Hamiltonian way of thermal perturbation, fourth-order Runge-Kutta method, Monte Carlo method, quantum perturbed way and thermodynamic and statistical method, and so on. In this review the numerical simulation results of bio-energy transport in uniform protein molecules, the influence of structure nonuniformity on the bio-energy transport, the effects of temperature of systems on the bio-energy transport and the simultaneous effects of structure nonuniformity, damping and thermal perturbation of proteins on the bio-energy transport in a single chains and helical molecules were included and studied, respectively. The results obtained from these studies and reviews represent that Davydov's soliton is really unstable, but Pang's soliton is stable at physiologic temperature $300 \mathrm{~K}$ and underinfluences of structure nonuniformity or disorder, side groups, imported impurities and damping of medium, which is consistent with analytic results. Thus we can still conclude that the soliton in Pang's model is exactly a carrier of the bio-energy transport, Pang's theory is appropriate to helical protein molecules.

\section{Introduction}

As it is well known, the so-called life is just processes of mutual changes and coordination of the bio-material, bio-energy and bio-information, their synthetic movements and cooperative changes are total life activity in the live systems in the light of biophysicist's view, where the bio-material is the foundation if life, the bio-energy is its center, the bio-information is the key of life activity, but the transformation and 
transfer of bio-information are always accompanied by the transport of bio-energy in living systems [1]. Thus, the bio-energy and its transport are an fundamental and important process in life activity. As a matter of fact, many biological processes, such as muscle contraction, DNA reduplication, neuroelectric pulse transfer on the neurolemma and work of calcium pump and sodium pump, and so on, are associated with bio-energy transport in the life bodies, where the energy is released by the hydrolysis of adenosine triphosphate (ATP). Namely, an ATP molecule reacts with water, which results in the energy release of $0.43 \mathrm{eV}$ under normal physiological conditions. The reaction can be represented by

$$
\mathrm{ATP}^{4-}+\mathrm{H}_{2} \mathrm{O} \rightarrow \mathrm{ADP}^{3-}+\mathrm{HPO}_{4}^{2-}+\mathrm{H}^{+}+0.43 \mathrm{eV}
$$

where ADP is the adenosine diphosphate. Just so, there are always a biological process of energy transport from production place to absorption place in the living systems. Therefore, investigation of bio-energy transport along protein molecules and determination its rules have an important significance in life science. In general, the bio-energy is transported along the protein molecules. However, understanding the mechanism of the transport in living systems has been a long-standing problem that remains of great interest up to now.

\section{Nonlinear excitation and theory of energy transport in protein molecules}

\section{Davydov theory of soliton excitation and its improvement}

Generally speaking, the energy can be converted to a particular vibrational excitation within a protein molecule. A likely recipient exchange is the amide-I vibration. Their vibration is primarily a stretch and contraction of the $\mathrm{C}=\mathrm{O}$ bond of the peptide groups. The amide-I vibration is also a prominent feature in infrared and Raman spectra of protein molecules. Experimental measurement shows that one of the fundamental frequencies of the amide-I vibration is about $0.205 \mathrm{eV}$. This energy is about half the energy released during the ATP hydrolysis. Moreover, it remains nearly constant from protein to protein, indicating that it is rather weakly coupled to other degrees of freedom. All these factors can lead to the assumption that the energy released by ATP hydrolysis might stay localized and stored in the amide-I vibration excitation. As an alternative to electronic mechanisms [2-4], one can assume that the energy is stored as vibrational energy of the $\mathrm{C}=0$ stretching mode (amide-I) in a protein polypeptide chain. Following Davydov's idea [5-12],ones take into account the coupling between the amide-I vibrarional quantum (exciton) and the acoustic phonon (molecular displacements) in the amino acid residues; Through the coupling, nonlinear interaction appears in the motion of the vibrartional quanta, which could lead to a self-trapped state of the vibrational quantum. The latter plus the deformational amino acid lattice together can travel over macroscopic distances along the molecular chains, retaining the wave shape, energy, momentum and other properties of the quasiparticle. In figure 1, structure of $\alpha$-helical protein this way, the bio-energy can be transported as a localized "wave packet" or soliton. This is just the Davydov's model of bio-energy transport in proteins, which was proposed in the 1970s [5-7].

Davydov model of bio-energy transport works at $\alpha$-helical proteins as shown in figure 1. Following Davydov idea [5-14, the Hamiltonian describing such system is of the form

$$
\begin{aligned}
& H_{D}=\sum_{n}\left[\varepsilon_{0} B_{n}^{+} B_{n}-J\left(B_{n}^{+} B_{n-1}+B_{n} B_{n+1}^{+}\right)\right]+\sum_{n}\left[\frac{P_{n}^{2}}{2 M}+\frac{1}{2} w\left(u_{n}-u_{n-1}\right)^{2}\right] \\
& +\sum_{n}\left[\chi_{1}\left(u_{n+1}-u_{n-1}\right) B_{n}^{+} B_{n}\right]=H_{a}+H_{p}+H_{i n t}
\end{aligned}
$$




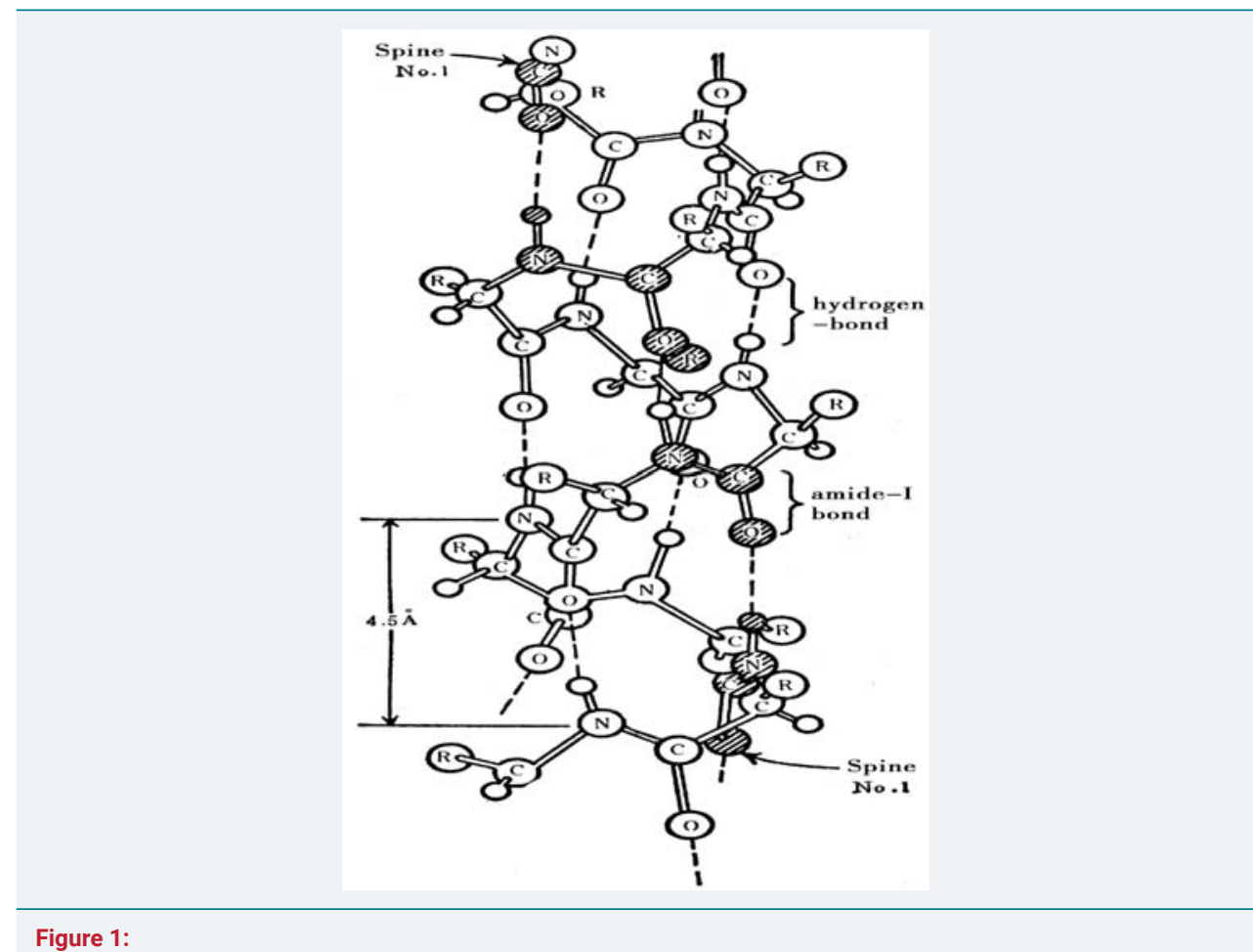

In Davydov's model, where $\varepsilon_{0}=0.205 \mathrm{ev}$ is the amide-I quantum energy, $-\mathrm{J}$ is the dipole-dipole interaction energy between neighbouring amides, $\mathrm{B}_{n}^{+}\left(\mathrm{B}_{\mathrm{n}}\right)$ is the creation (annihilation) operator for an amide-I quantum (exciton) in the site $n, u_{n}$ is the displacement operator of lattice oscillator at site $n, \mathrm{P}_{\mathrm{n}}$ is its conjugate momentum operator, $\mathrm{M}$ is the mass of an amino acid residue, $\mathrm{w}$ is the elasticity constant of the protein molecular chains, and $\chi_{1}$ is an nonlinear coupling parameter and represents the coupling size of the exciton- phonon interaction in the protein molecules. The wave function of states of the systems in Davydov model is of the form [5-14]:

$$
\begin{aligned}
& \left|\mathrm{D}_{2}(\mathrm{t})=\right| \varphi_{\mathrm{D}}(\mathrm{t})>1 \beta(\mathrm{t})>=\sum_{n} \phi_{n}(t) B_{n}^{+} \exp \left(-\frac{i}{\hbar} \sum_{n}\left[\beta_{n}(t) P_{n}-\pi_{n}(t) u_{n}\right]\right) 10>. \\
& \text { or } \mid D_{1}(t)>=\sum_{n}\left\{\phi_{n}(t) B_{n}^{+} \exp \left(\sum_{q}\left[\alpha_{n q}(t) a_{q}^{+}-\alpha_{n q}^{*}(t) a_{n}\right]\right)\right\} 10>
\end{aligned}
$$

where $\mathrm{I} 0>=\mathrm{I} 0>_{\mathrm{ex}} \mathrm{I} 0>_{\mathrm{ph}}, \mathrm{I} 0>_{\mathrm{ex}}$ and $\mathrm{I} 0>_{\mathrm{ph}}$ are the ground states of the exciton and phonon, respectively, $\mathrm{a}_{\mathrm{q}}\left(\mathrm{a}_{\mathrm{q}}^{+}\right)$is annihilation (creation) operator of the phonon with ware vector q, $\varphi_{n}(t)$ and $\beta_{\mathrm{n}}(t)=<D_{2}\left|u_{n}\right| D_{2}>$ and $\pi_{n}(t)=<D_{2}\left|P_{n}\right| D_{2}>$ and

$\alpha_{\mathrm{q}}(\mathrm{t}) \leqslant \mathrm{D}_{1}(\mathrm{t})\left|\mathrm{a}_{\mathrm{q}}\right| \mathrm{D}_{1}(\mathrm{t})>$ are some undetermined functions of time. The Davydov soliton obtained from Eqs.(1)-(2) in the semiclassical limit and using the continuum approximation has the from of

$$
\left.\varphi_{\mathrm{D}}(\mathrm{x}, \mathrm{t})=\left(\frac{\mu_{\mathrm{D}}}{2}\right)^{1 / 2} \operatorname{sech}\left[\frac{\mu_{\mathrm{D}}}{\mathrm{r}_{0}}\left(\mathrm{x}-\mathrm{x}_{0}-\mathrm{t}\right)\right] \exp \left\{\mathrm{i}\left[\frac{\hbar \mathrm{v}}{2 \mathbf{J}_{0}^{2}}\left(\mathrm{x}-\mathrm{x}_{0}\right)-\mathrm{E}_{\mathrm{v}} \mathrm{t} / \hbar\right)\right]\right\}
$$

which corresponds to an excitation localized over a scale $\mathrm{r} 0 / \mu_{\mathrm{D}}$, where $\mu_{D}=\chi_{1}^{2} /\left(1-s^{2}\right) w J, \quad \mathrm{G}_{\mathrm{D}}=4 J \mu_{D}, \quad \mathrm{~s}^{2}=v^{2} / v_{0}^{2}, \quad \mathrm{v}_{0}=r_{0}(\mathrm{w} / \mathrm{M})^{1 / 2}$ is the sound speed $\mathrm{i}_{\mathrm{n}}$ the protein molecular chains, $\mathrm{v}$ is the velocity of the soliton, $\mathrm{r}_{0}$ is the lattice constant. Evidently, the soliton contains only one exciton because $\mathrm{N}=<\phi_{D}(t)|\hat{N}| \phi_{D}(t)>=1$. This shows that the Davydov soliton is formed through self-trapping of one exciton, its 
binding energy is $E_{B D}=-\chi_{1}^{4} / 3 \mathrm{Jw}^{2}$.

Davydov's idea yields a compelling picture for the mechanism of bioenergy transport in protein molecules and consequently has been the subject of a large number of works [15-103]. A lot of issues related to the Davydov model, including the foundation and accuracy of the theory, the quantum and classical properties and the thermal stability and lifetimes of the Davydov soliton have extensively been studied by many scientists. However, considerable controversy has arisen in recent years concerning whether the Davydov soliton is sufficiently stable in the region of biological temperature to provide a viable explanation for bio-energy transport. It is out of question that the quantum fluctuations and thermal perturbations are expected to cause the Davydov soliton to decay into a delocalized state. Some numerical simulations indicated that the Davydov soliton is not stable at the biological temperature 300K [36-49]. Other simulations showed that the Davydov soliton is stable at 300K [36-49], but they were based on classical equations of motion which are likely to yield unreliable estimates for the stability of the soliton [2-4]. The simulations based on the $\mathrm{ID}_{2}>$ state in Eq.(2) generally show that the stability of the soliton decreases with increasing temperatures and that the soliton is not sufficiently stable in the region of biological temperature. Since the dynamical equations used in the simulations are not equivalent to the nonlinear Schrödinger equation, the stability of the soliton obtained by these numerical simulations is unavailable or unreliable. The simulation[9] based on the $\mathrm{ID}_{1}>$ state in Eq. (3) with the thermal treatment of Davydov [15-20] , where the equations of motion are derived from a thermally averaged Hamiltonian, yields the confusing result that the stability of the soliton is enhanced with increasing temperature, predicting that $\mathrm{ID}_{1}>-$ type soliton is stable in the region of biological temperature. Evidently, the conclusion is doubtful because the Davydov procedure in which an equation of motion for an average dynamical state obtained from an average Hamiltonian, corresponding to the Hamiltonian averaged over a thermal distribution of phonons, is inconsistent with standard concepts of quantum-statistical mechanics in which a density matrix must be used to describe the system. Therefore, any fully exact quantum- mechanical treatment for the numerical simulation of the Davydov soliton does not exist. However, for the thermal equilibrium properties of the Davydov soliton, there is a quantum Monte Carlo simulation $[64,65]$. In this study the correlation characteristic of solitonlike quasiparticles occur only at low temperatures, about $\mathrm{T}<10 \mathrm{k}$ for widely accepted parameter values. This is consistent at a qualitative level with the result of Cottingham et al. [66,67]. The latter is a straightforward quantum- mechanical perturbation calculation, the lifetime of the Davydov soliton obtained by using this method is too small (about $10^{-12}-10^{-13} \mathrm{sec}$ ) to be useful in biological processes. This indicates clearly that the Davydov solution is not a true wave function of the systems. A through study in terms of parameter values, different types of nonuniformity, different thermalization schemes, different wave functions, and different associated dynamics leads to a very complicated picture for the Davydov model [44-63]. These results do not completely rule out the Davydov theory, however they do not eliminate the possibility of another wave function and a more sophisticated Hamiltonian of the system having a soliton with longer lifetimes and good thermal stability.

Indeed, the question of the lifetime of the soliton in protein molecules is twofold. In Langevin dynamics, the problem consists of uncontrolled effects arising from the semiclassical approximation. In quantum treatments, the problem has been the lack of an exact wave function for the soliton. The exact wave function of the fully quantum Davydov model has not been known up to now. Different wave functions have been used to describe the states of the fully quantum-mechanical systems [21-32]. Although some of these wave functions lead to exact quantum states and exact quantum dynamics in the $\mathrm{J}=0$ state, they also share a problem with the original Davydov wave function, namely that the degree of approximation included when $\mathrm{J} \neq 0$ is not known. 
Therefore, it is necessary to reform Davydov's wave function. Scientists had though that the soliton with a multiquantum $n \geq 2$, for example, the coherent state of Brown et al. [21-28], the multiquantum state of Kerr et al. [62,63] and Schweitzer et al. [66,67], the two- quantum state of Cruzeiro-Hansson [44-49] and Forner [74], and so on, would be thermally stable in the region of biological temperature and could provide a realistic mechanism for bio-energy transport in protein molecules. However, the assumption of the standard coherent state is unsuitable or impossible for biological protein molecules because there are innumerable particles in this state and one could not retain conservation of the number of particles of the system. The assumption of a multiquantum state $(n>2)$ along with a coherent state is also inconsistent with the fact that the bioenergy released in ATP hydrolysis can excite only two quanta of amide-I vibration. On the other hand, the numerical result shows that the soliton of two-quantum state is more stable than that with a one-quantum state [74].

Cruzeiro-Hansson [44-49] had thought that Forner's two-quantum state in the semiclassical case was not exact. Therefore, he constructed again a so-called twoquantum state for the semiclassical Davydov system as follows [44-49]:

$$
|\phi(t)\rangle=\sum_{n, m=1} \phi_{n m}\left(\left\{\mathrm{u}_{1}\right\},\left\{\mathrm{P}_{1}\right\}, \mathrm{t}\right) \mathrm{B}_{\mathrm{n}}^{+} \mathrm{B}_{\mathrm{m}}^{+} \mid 0>_{\mathrm{ex}}
$$

where $B_{n}\left(B_{n}^{+}\right)$is the annihilation (creation) operator for an amide-I vibration quantum (exciton), $\mathrm{u}_{1}$ is the displacement of the lattice molecules, $\mathrm{P}_{1}$ is its conjugate momentum, and $\mid 0>_{e x}$ is the ground state of the exciton. He calculated the average probability distribution of the exciton per site, and average displacement difference per site, and the thermodynamics average of the variable, $P=B_{1}^{+} B_{1}-B_{2}^{+} B_{2}$, as a measure of localization of the exciton, versus quantity $v=W / \chi_{1}^{2}$ and $h \beta\left(\beta=1 / k_{B} T\right)$ in the socalled two-quantum state, Eq.(5), where $\chi_{1}$ is a nonlinear coupling parameter related to the interaction of the exciton-phonon in the Davydov model. Their energies and stability are compared with those of the one-quantum state. From the results of above thermal averages, he drew the conclusion that the wave function with a two-quantum state can lead to more stable soliton solutions than that with a one-quantum state, and that the usual Langevin dynamics, whereby the thermal lifetime of the Davydov soliton is estimated, must be viewed as underestimating the soliton lifetime.

However, by checking carefully Eq.(5), Pang found that the Cruzeiro-Hansson wave function does not represent exactly the two-quantum state. To find out how many quanta the state Eq.(5) indeed contains, the expectation value of the exciton number operator $\hat{N}=\sum_{n} B_{n}^{+} B_{n}$ has to be computed in this state Eq.(5), then the exciton numbers $\mathrm{N}$ contained are

$$
\begin{aligned}
\mathrm{N} & =\left\langle\varphi\left|\sum_{\mathrm{n}} \mathrm{B}_{\mathrm{n}}^{+} \mathrm{B}_{\mathrm{n}}\right| \varphi>=\sum_{\mathrm{ijlmn}} \varphi_{\mathrm{im}}^{*} \varphi_{\mathrm{j} 1 \text { ex }}<0\left|\mathrm{~B}_{\mathrm{i}} \mathrm{B}_{\mathrm{m}} \mathrm{B}_{\mathrm{n}}^{+} \mathrm{B}_{\mathrm{n}} \mathrm{B}_{\mathrm{j}}^{+} \mathrm{B}_{1}^{+}\right| 0>_{\mathrm{ex}}\right. \\
& =\sum_{\mathrm{nj}}\left(\varphi_{\mathrm{nj}}^{*} \varphi_{\mathrm{jn}}+\varphi_{\mathrm{jn}}^{*} \varphi_{\mathrm{jn}}\right)+\sum_{\mathrm{nl}}\left(\varphi_{\mathrm{nl}}^{*} \varphi_{\mathrm{nl}}+\varphi_{\mathrm{ln}}^{*} \varphi_{\mathrm{nl}}\right)=4
\end{aligned}
$$

Therefore, the state, Eq.(5), as it is put forward in Ref.[44-49],deals with four excitons (quanta), instead of two excitons in contradiction to the author's statements. Obviously, it is impossible to create the four excitons by the energy released in the ATP hydrolysis (about $0.43 \mathrm{eV}$ ). Thus the author's wave function in Eq.(5) is still not relevant to protein molecules, and his discussion and conclusion are unreliable and implausible in that paper [44-49].

It is believed that the physical significance of the wave function, Eq.(5), is also unclear, or at least is very difficult to understand. As far as the physical meaning of Eq.(5) is concerned, it represents only a combinational state of single-particle excitation with two quanta created at sites $n$ and $m ; \varphi_{\mathrm{m}}\left(\left\{\mathrm{u}_{1}\right\}\left\{\mathrm{P}_{1}\right\}\right.$ t $)$ is the probability amplitude of particles occurring at the sites $n$ and $m$ simultaneously. In general, $n=\neq m$ 
and $\varphi_{\mathrm{m}} \neq \varphi_{\mathrm{n}} \varphi_{\mathrm{m}}$ in accordance with the author's idea. In such a case it is very difficult to imagine the form of the soliton by the mechanism of self- trapping of the two quanta under the action of the nonlinear exciton-phonon interaction, especially when the difference between $n$ and $m$ is very large .Meanwhile, Hansson has also not explained the physical and biological reasons and the meaning for the proposed trial state. Therefore, we think that the Cruzeiro-Hansson representation is still not an exact wave function suitable for protein molecules. Thus, the wave function of the systems is still an open problem today, a correct theory of bio-energy transport need still to construct further.

\section{Pang's model of soliton excitation and properties of energy transport}

From serious study of Davydov model we can find that it is indeed too simple, i.e., it does not denote the elementary properties of the collective excitations occurring in protein molecules, and many improvements of it have also been unsuccessful. In fact, Davydov operation is not strictly correct. A basic reason for the failure of the Davydov model is just that it ignores completely the above important properties of the protein molecules. Let us consider the Davydov model with the present viewpoint. First, as far as the Davydov wave functions, both $\mid D_{1}>$ and $\mid D_{2}>$, are concerned, they are not true solutions of the protein molecules. On the one hand, there is obviously asymmetry in the Davydov wave function since the phononic parts is a coherent state, while the excitonic part is only an excitation state of a single particle. It is not reasonable that the same nonlinear interaction generated by the coupling between the excitons and phonons produces different states for the phonon and exciton. Thus, Davydov's wave function should be modified [72-103], i.e., the excitonic part in it should also be coherent or quasicoherent to represent the coherent feature of collective excitation in protein molecules. However, the standard coherent [21-28] and large-n excitation states $[62,63]$ are not appropriate to the protein molecules due to the reasons mentioned above. Similarly, Forner's and Cruzeiro-Hansson's two-quantum states do not fulfill the above request.

On the basis of the work of Cruzeio-Hansson, Forner, Schweitzer and Takeno and Pang, both the Hamiltonian and the wave function of the Dovydov model have been improved and developed by Pang [104-125], in which Davydov's wave function has been replaced with a quasi-coherent two-quanta state for exhibiting the coherent behaviors of collective excitations [126-129] which are a feature of the energy released in ATP hydrolysis in the systems. The new wave function is represented [104-125] by

$$
\begin{aligned}
& |\Phi(t)>=| \varphi(t)>\left|\beta(t)>=\frac{1}{\lambda}\left[1+\sum_{n} \varphi_{n}(t) B_{n}^{+}+\frac{1}{2 !}\left(\sum_{n} \varphi_{n}(t) B_{n}^{+}\right)^{2}\right]\right| 0>_{\text {ex }} \\
& \times \exp \left\{-\frac{i}{\hbar} \sum_{n}\left[\beta_{n}(t) P_{n}-\pi_{n}(t) u_{n}\right]\right\} \mid 0>_{p h}
\end{aligned}
$$

where $B_{n}^{+}$and $B_{n}$ are boson creation and annihilation operators for the exciton, $\mid 0>_{e x}$ and $\mid 0>_{p h}$ are the ground states of the exciton and phonon, respectively $u_{n}$ and $P_{n}$ are the displacement and momentum operators of the lattice oscillator at site $n$, respectively. The $\varphi_{n}(t), \beta_{n}(t)=<\Phi(t)\left|u_{n}\right| \Phi(t)>$ and $\pi_{n}(t)=<\Phi(t)\left|P_{n}\right| \Phi(t)>$ are three sets of unknown functions, $\lambda$ is a normalization constant. It is assumed hereafter that $\lambda=1$ for convenience of calculation, except when explicitly mentioned.

A second problem arises from the Davydov Hamiltonian [5-14]. The Davydov Hamiltonian takes into account the resonant or dipole-dipole interaction of the neighboring amide-I vibrational quanta in neighboring peptide groups with an electrical moment of about 3.5D, but why do we consider not the changes of relative displacement of the neighboring peptide groups arising from this interaction ? This means that it is reasonable to add the new interaction term $\chi_{2}\left(u_{n+1}-u_{n}\right)\left(B_{n+1}^{+} B_{n}+B_{n}^{+} B_{n+1}\right)$ 
into the Davydov's Hamiltonian for representing the correlations of the collective excitations and collective motions in the protein molecules, as mentioned above [76103]. Although the dipole- dipole interaction is small as compared with the energy of the amide-I vibrational quantum, the change of relative displacement of neighboring peptide groups resulting from this interaction cannot be ignored due to the sensitive dependence of the dipole-dipole interaction on the distance between amino acids in the protein molecules, which is a kind of soft condensed matter and bio-self-organization. Thus, the Davydov Hamiltonian is replaced by

$$
\begin{aligned}
& H=H_{e x}+H_{p h} H_{\text {üiü }}=\sum_{n}\left[\varepsilon B_{n}^{+} B_{n}-J\left(B_{n}^{+} B_{n+}+B_{n} B_{n+}^{+}\right)\right]+\sum_{n}\left[\frac{P_{n}^{2}}{2 M}+\frac{1}{2} w\left(u_{n}-u_{n-}\right)^{2}\right] \\
& +\sum_{n}\left[\chi_{\text {iulut }}\left(u_{n+}-u_{n-}\right)\right] B_{n}^{+} B_{n}+\chi\left(u_{n+}-u_{n}\right) \times\left(B_{n+}^{+} B_{n}+B_{n}^{+} B_{n+}\right)
\end{aligned}
$$

Where $\varepsilon_{0}=0.205 \mathrm{eV}$ is the energy of the exciton (the $\mathrm{C}=0$ stretching mode). The present nonlinear $\backslash$ coupling constants are $\chi_{1}$ and $\chi_{1}$. They represent the modulations of the on-site energy and resonant (or dipole-dipole) interaction energy of excitons caused by the molecules displacements, respectively. $M$ is the mass of a amino acid molecule and $w$ is the elasticity constant of the protein molecular chains. J is the dipoledipole interaction energy between neighboring sites. The physical meaning of the other quantities in Eq.(7) are the same as those in the above explanations.

The Hamiltonian and wave function shown in Eqs.(6)-(7) are different from Davydov's. We add a new interaction term, $\sum_{n} \chi_{2}\left(u_{n+1}-u_{n}\right)\left(B_{n+1}^{+} B_{n}+B_{n}^{+} B_{n+1}\right)$, into the original Davydov Hamiltonian. Thus the Hamiltonian now has better one-by one correspondence of the interactions and can represent the features of mutual correlations of the collective excitations and of collective motions in the protein molecules. However, we here should point out that the different coupling between the relevant modes was also considered by Takeno et al. [75-77,130-131] and Pang [78103] in the Hamiltonian of the vibron-soliton model for one-dimensional oscillatorlattice and protein systems, respectively, but the wave functions of the systems they used are different from Eqs.(6)-(7).

Obviously, the present wave function of the exciton in Eq.(6) is not an excitation state of single particle, but rather a coherent state, more precisely, a quasicoherent state, because it retain only fore three terms of the expansion of a standard coherent state, which thus can be viewed as an effective truncation of a standard coherent state. It is clear that when small $\phi_{n}(t)$, i.e.,

$\phi_{n}(t) \ll<1$, we can represent the wave function of the excitons, $\phi_{n}(t)>$, in Eq. $(6)[104-$ 125] by

$$
\begin{aligned}
& \left|\varphi(t)>=\left[1+\sum_{n} \varphi_{n}(t) B_{n}^{+}+\frac{1}{2 !}\left(\sum_{n} \varphi_{n}(t) B_{n}^{t}\right)^{2}\right]\right| 0>_{e x} \sim \exp \left[-\frac{1}{2} \sum_{n}\left|\varphi_{n}(t)\right|^{2}\right] \times \\
& \exp \left\{\sum_{n} \varphi_{n}(t) B_{n}^{+}\right\}\left|0>_{e x}=\exp \left\{\sum_{n}\left[\varphi_{n}(t) B_{n}^{+}-\varphi_{n}^{*}(t) B_{n}\right]\right\}\right| 0>_{e x}
\end{aligned}
$$

The last representation in Eq.(8) is a standard coherent state. Therefore, the state of exciton denoted by the wave function $\varphi(t)>$ has a coherent feature. In the meanwhile, we can verify that the new wave function in Eq.(6) is also normalized at $\lambda=1$. Since the condition of $\sum_{n}\left|\varphi_{n}(t)\right|^{2}=1$ is required in the calculation, then the above condition of $\phi_{n}(t)<<1$ also is naturally satisfied for the protein molecules consisting of several hundreds or thousands of amino acid residures. Thus the above representation in Eq.(8) is justified and correct for the protein molecules. Since the coherent state is certainly normalized, then the wave function $\phi(t)>$ in Eq.(6), which can be represented as a standard coherent state, is exactly normalized at $\lambda=1$. Clearly, the above demonstration 
for the normalization is correct, reasonable and credible because it is obtained from a strict mathematical, physical and biological theory. Thus we have not any reasons to doubt the normalization of wave function in Eq.(6). However, it is not an eigenstate of the number operator because of

$$
\left.\left.\hat{N}\left|\varphi(t)>=\sum_{n} B_{n}^{+} B_{n}\right| \varphi(t)\right\rangle=\left\{\sum_{n} \varphi_{n}(t) B_{n}^{+}+\left(\sum_{n} \varphi_{n}(t) B_{n}^{+}\right)^{2}\right\}\left|0>_{e x}=2\right| \varphi(t)\right\rangle-\left(2+\sum_{n} \varphi_{n}(t) B_{n}^{+}\right) \mid 0>_{e x}
$$

However, in this state the numbers of quanta are determinate instead of innumerable. Since the third term in the exciton part in Pang's wave function contains the coefficient of " $1 / 2$ !", which guarantees that the third term in the exciton's wave function contribute only one quantum, then we find that the state contains number of exciton by computing the expectation value of the number operator $N=\sum_{n} B_{n}^{+} B_{n}$ in this state and sum over the states, i.e.,

$$
\begin{aligned}
& N=<\varphi(t)|\hat{N}| \varphi(t)>=\sum_{n}<\varphi(t)\left|B_{n}^{+} B_{n}\right| \varphi(t)>=\left\{\sum_{n}\left|\varphi_{n}(t)\right|^{2}+\left(\sum_{n}\left|\varphi_{n}(t)\right|^{2}\right)\left(\sum_{m}\left|\varphi_{m}(t)\right|^{2}\right)\right\} \\
& =\left(\sum_{n}\left|\varphi_{n}(t)\right|^{2}\right)\left(1+\sum_{m}\left|\varphi_{m}(t)\right|^{2}\right)=2
\end{aligned}
$$

Therefore, $\mid \varphi(t)>$ contains only two quanta, instead of one quantum or three quanta, i.e., it represents exactly a coherent superposition of the excitonic state with two quanta and the ground state of the exciton. Thus the new wave function not only exhibits the coherent feature of the collective excitation of excitons and phonons caused by the nonlinear interaction generated by virtue of the exciton-phonon interaction, which makes the wave function of the states of the system symmetrical, but also agrees with the fact that the energy released in the ATP hydrolysis (about $0.43 \mathrm{eV}$ ) can only create two amide-I vibrational quanta which, thus, can also make the numbers of excitons maintain conservation in the Hamiltonian, Eq.(7).Then it is correct and available. We here refer to it as a two quanta quasicoherent state. Obviously, it is completely different from Davydov's, which is an excitation state of a single particle with one quantum and an eigenstate of the number operator. At the same time, the new wave function in Eq.(6) is either two- quanta states proposed by Forner [74] and Cruzeiro-Hansson [44-49] or a standard coherent state proposed by Brown et al. [21-28], and Kerr et al. [62,63,] and Schweitzer et al's [66-67] multiquanta states. Therefore, the wave function, Eq.(6), is new for the protein molecular systems. In the meanwhile, the new wave function has the following advantages, i.e., the equation of motion of the soliton in the system can also be obtained from the Heisenberg equations of the creation and annihilation operators in quantum mechanics using Eqs.(6) and (7). However, it is improssible for the wave function of state of the system in other models, including the one-quanta state [5-14] and the two-quanta state [44-49]. Therefore, the above Hamitonian and wave function, Eqs.(6) and (7),are both new and appropriate to the protein molecules.

We know fron Eq.(6) that the phonon part depending on the displacement and momentum operators in the new wave function in Eq.(6) is a coherent state of the normal model creation and annihilation operators. A coherent state for the mode with wave vector $q$ is denoted by [5-14,62-63,104-124]

$$
\begin{aligned}
& |\alpha(t)\rangle=\exp \left(\sum_{q}\left[\alpha_{q}(t) a_{q}^{+}-\alpha_{q}^{*}(t) a_{q}\right]\right)|0\rangle_{p h} \text {. Utilizing the standard transformations: } \\
& u_{n}=\sum_{q}\left[\frac{\hbar}{2 M \omega_{q}}\right]^{1 / 2} e^{i q n r_{0}}\left(a_{-q}^{+}+a_{q}\right), P_{n}=i \sum_{q}\left[\frac{M \hbar \omega_{q}}{2 N}\right]^{1 / 2} e^{i q n r_{0}}\left(a_{-q}^{+}-a_{q}\right)
\end{aligned}
$$

we can get $|\alpha(t)\rangle=|\beta(t)\rangle$, where $|\beta(t)\rangle$ is same in Eq.(6) and $\omega_{q}=2(w / M)^{1 / 2} \sin \left(r_{0} q / 2\right)$ $[62,63], r_{0}$ is the distance between neighboring amino acid molecules, and $a_{q}\left(a_{q}^{+}\right)$is the annihilation (Creation) operator of the phonon with wave vector $q$. Utilizing the 
above results and the formulas of the expectation values of the Heisenberg equations of operators, $u_{n}$ and $P_{n}$, in the state $|\Phi(t)\rangle$.

$$
\begin{aligned}
& i \hbar \frac{\partial}{\partial t}\left\langle\Phi(t)\left|u_{n}\right| \Phi(t)\right\rangle=\left\langle\Phi ( t ) \left[\left[u_{n}, H\right]|\Phi(t)\rangle\right.\right. \\
& i \hbar \frac{\partial}{\partial t}\left\langle\Phi(t)\left|P_{n}\right| \Phi(t)\right\rangle=\left\langle\Phi(t)\left[u_{n}, H\right] \Phi(t)\right\rangle \text { and }
\end{aligned}
$$

the time-dependent Shrodinger equation [79-105], i $\frac{\partial}{\partial t}|\Phi(t)\rangle=H|\Phi(t)\rangle$, in the continuum approximation we get we can obtain:

$$
i \hbar \frac{\partial}{\partial t} \phi(x, t)=R(t) \phi(x, t)-J r_{0}^{2} \frac{\partial^{2}}{\partial x^{2}} \phi(x, t)-G_{p}|\phi(x, t)|^{2} \phi(x, t)
$$

and

$$
M \frac{\partial^{2} \beta(x, t)}{\partial t^{2}}-w r_{o}^{2} \frac{\partial^{2} \beta(x, t)}{\partial x^{2}}=-4\left(\chi_{1}+\chi_{1}\right) r_{0} \frac{\partial}{\partial x}|\phi(x, t)|^{2}
$$

here

$$
R(t)=\varepsilon_{0}-2 J+\frac{5}{2}\left\{W(t)-\frac{1}{2} \sum_{m}\left[\dot{\beta}_{m}(t) \pi_{m}(t)-\dot{\pi}_{m}(t) \beta(t)\right]\right\}
$$

and

$$
\begin{gathered}
W(t)=\left\langle\beta(t)\left|H_{\text {ph }}\right| \beta(t)\right\rangle, \\
\sum_{n}\left(\frac{1}{2 M} \pi_{n}^{2}(t)+\frac{1}{2} w\left[\beta_{n}(t)-\beta_{n-1}(t)\right]^{2}\right)+\sum_{q} \frac{1}{2} \hbar \omega_{q} .
\end{gathered}
$$

The soliton solution of Eq.(11)[130-132] is thus

$$
\begin{aligned}
& \phi(x, t)=\left(\frac{\mu_{p}}{2}\right)^{1 / 2} \sec h\left[\left(\mu_{p} / r_{0}\right)\left(x-x_{0}-v t\right)\right] \times \exp \left\{i\left[\frac{\hbar v}{2 J r_{0}^{2}}\left(x-x_{0}\right)-E_{v} \frac{t}{\hbar}\right]\right\} \\
& \text { with } \mu_{P}=\frac{2\left(\chi_{1}+\chi_{2}\right)^{2}}{w\left(1-s^{2}\right) J}, s=v / v_{0}, G_{P}=\frac{8\left(\chi_{1}+\chi_{2}\right)^{2}}{w\left(1-s^{2}\right)}
\end{aligned}
$$

The above treatment yields a localized coherent structure with size of order $2 \pi$ $\mathrm{r}_{0} / \mu_{\mathrm{p}}$ that propagates with velocity $\mathrm{v}$ and can transfer energy $\mathrm{E}_{\mathrm{s01}}<2 \varepsilon_{0}$. Unlike bare excitons that are scattered by the interactions with the phonons, this soliton state describes a quasi-particle consisting of the two excitons plus a lattice deformation and hence a priori includes interaction with the acoustic phonons. So the soliton is not scattered and can spread through maintaining its form, energy, momentum and other quasiparticle properties after moving over a macroscopic distance. The bell-shaped form of the soliton in Eq.(13) also does not depend on the excitation method. It is selfconsistent. Since the soliton always move with velocity $v$ less than that of longitudinal sound $\mathrm{v}_{0}$ in the chains then they do not emit phonons, i.e., its kinetic energy is not transformed into thermal energy. This is one important reason for the high stability of the new soliton. In addition the energy of the soliton state is below the bottom of the bare exciton bands, the energy gap between then being $4 \mu_{\mathrm{p}}^{2} \mathrm{~J} / 3$ for small velocity of propagation. Hence there is an energy penalty associated with the destruction with transformation from the soliton state to a bare exciton state, i.e, the destruction of the soliton state requires simultaneous removal of the lattice distortion. We know that the transition probability to a lattice state without distortion is very small, in general, being negligible for a long chain. Considering this it is reasonable to assume that such a soliton is stable enough to propagate through the length of a typical protein structure. However, the thermal stability of the soliton state must be calculated quantitatively. The following calculation addresses this point explicitly. 
Although forms of the above equations of motion and the corresponding solution, Eqs.(11)- (13),are quite similar to those of the Davydov soliton, the properties of the new soliton have very large differences from the latter because the parameter values in the equation of motion and the solution Eqs.(11) and (13),including $R(t), G_{p}$ and $\mu_{p^{\prime}}$ have obvious distinctions from those in the Davydov model. A straightforward result of the new model is to increase the nonlinear interaction energy $G_{p}\left(G_{p}=2 G_{D} \times\right)$ $\left.\left[1+2\left(\chi_{2} / \chi_{1}\right)+\left(\chi_{2} / \chi_{1}\right)^{2}\right]\right)$ and the amplitude of the new soliton, and decrease its width due to an increase of $\mu_{p}\left(\mu_{p}=2 \mu_{D}\left[1+2\left(x_{2} / x_{1}\right)+\left(x_{2} / x_{1}\right)^{2}\right]\right)$ when compared with Davydov soliton, where $\mu_{D}=x_{1}^{2} / w\left(1-s^{2}\right) J$ and $G_{D}=4 x_{1}^{2} / w\left(1-s^{2}\right)$ are the corresponding values in Davydov model. Thus the localized feature of the new soliton is enhanced. Therefore, its stability against the quantum fluctuation and thermal perturbations is increased considerably as compared with the Davydov soliton.

The energy of the new soliton in Eq.(13) in the improved model can be represented [130-132] by

$$
\begin{aligned}
& E=<\Phi(t)|H| \Phi(t)>=\frac{1}{r_{0}} \int_{-\infty}^{\infty} 2\left[J r_{0}^{2}\left(\frac{\partial \phi}{\partial x}\right)^{2}+R|\phi(x, t)|^{2}-G_{p}|\phi(x, t)|^{4} d x+\right. \\
& \frac{1}{r_{0}} \int_{-\infty}^{\infty} \frac{1}{2}\left[M\left(\frac{\partial \beta(x, t)}{\partial t}\right)^{2}+w r_{0}^{2}\left(\frac{\partial \beta(x, t)}{\partial x}\right)^{2}\right] d x=E_{0}+\frac{1}{2} M_{\text {sol }} v^{2}
\end{aligned}
$$

The rest energy of the new soliton is $E_{0}=2\left(\varepsilon_{0}-2 J\right)-\frac{8\left(x_{1}+x_{2}\right)^{4}}{3 w^{2} J}=E_{s}^{0}+W$,

where $W=\left[2\left(x_{1}+x_{2}\right)^{4}\right] / 3 w^{2} J$ is the energy of deformation of the amono acid lattice. The effective mass of the new soliton is $\boldsymbol{M}_{\text {sol }}=2 \boldsymbol{m}_{e x}+\frac{8\left(\boldsymbol{x}_{1}+\boldsymbol{x}_{2}\right)^{4}\left(9 \boldsymbol{s}^{2}+2-3 \boldsymbol{s}^{4}\right)}{3 \boldsymbol{w}^{2} \boldsymbol{J}\left(1-\boldsymbol{s}^{2}\right)^{3} \boldsymbol{v}_{0}^{2}}$.

In such a case, the binding energy of the new soliton is

$$
\boldsymbol{E}_{B P}=\frac{-8\left(\boldsymbol{x}_{1}+\boldsymbol{x}_{2}\right)^{4}}{3 \boldsymbol{J} \boldsymbol{w}^{2}}=8 \boldsymbol{E}_{\boldsymbol{B D}}\left[1+4\left(\frac{\boldsymbol{x}_{2}}{\boldsymbol{x}_{1}}\right)+6\left(\frac{\boldsymbol{x}_{2}}{\boldsymbol{x}_{1}}\right)^{2}+4\left(\frac{\boldsymbol{x}_{2}}{\boldsymbol{x}_{1}}\right)^{3}+\left(\frac{\boldsymbol{x}_{2}}{\boldsymbol{x}_{1}}\right)^{4}\right]
$$

$E_{B P}$ is larger than that of the Davydov soliton.The latter is $\boldsymbol{E}_{B D}=-\boldsymbol{x}_{1}^{4} / 3 \boldsymbol{J} \boldsymbol{w}^{2}$. We can estimate that the binding energy of the new soliton is about several decades larger than that of the Davydov soliton .This is a very interesting result. It is helpful to enhance thermal stability of the new soliton. Obviously, the increase of the binding energy of the new soliton comes from its two-quanta nature and the added interaction, $\sum_{i} \chi_{2}\left(\boldsymbol{u}_{n+1}-\boldsymbol{u}_{n}\right)\left(\boldsymbol{B}_{n+1}^{+} \boldsymbol{B}_{n}+\boldsymbol{B}_{n}^{+} \boldsymbol{B}_{n+1}\right)$, in the Hamiltonian of the systems, Eq. (7). However, we see from Eq.(16) that the former plays the main role in the increase of the binding energy and the enhancement of thermal stability for the new soliton relative to the latter due to $\chi_{2<1}$. The increase of binding energy results in a significant change of property of the new soliton, which are discussed as follows.

In comparing various correlations to this model, it is helpful to consider them as a function of a composite coupling parameter like that of Young et al. [133], and Scott [15-20], that can be written as

$4 \pi \alpha_{P}=\left(\chi_{1}+\chi_{2}\right)^{2} / 2 w \hbar \omega_{D}$, where $\omega_{D}=(\boldsymbol{w} / \boldsymbol{M})^{1 / 2}$ is the band edge for acoustic phonons (or Debye frequency). If $4 \pi \alpha_{p}<<1$, it is said to be weak. Using widely accepted values for the physical parameters in the alpha helix protein molecule [5-125]:

$$
\begin{aligned}
& \boldsymbol{J}=1.55 \times 10^{-22} \boldsymbol{J} . \boldsymbol{w}=(13-19.5) \mathbf{N} / \mathbf{m} . \boldsymbol{M}=(1.17-1.91) \times 10^{-25} \mathbf{k g} \\
& \chi_{1}=62 \times 10^{-12} \boldsymbol{N} . \quad \chi_{2}=(10-18) \times 10^{-12} \boldsymbol{N} . \boldsymbol{r}_{0}=4.5 \times 10^{-10} \mathbf{m} .
\end{aligned}
$$


we can estimate that the coupled constant lies in the region of $4 \pi \alpha_{p}=0.11-0.273$, but $4 \alpha_{D=} 0.036-0.045$ for the Davydov model. Hence, the new model is not a weakly coupled theory as compared with the Davydov model. Using again the notation of Venzel and Fischer [134], Nagy [135] and Wagner and Kongeter [136], it is convenient to define another composite parameter [15-20]: $\gamma=J / 2 \hbar w_{D}$. In terms of the two composite parameters, $4 \pi \alpha_{p}$ and ${ }_{\gamma}$, the soliton binding energy in the new model can be written by

$$
\boldsymbol{E}_{\boldsymbol{B P}} / \boldsymbol{J}=8\left(4 \pi \alpha_{\boldsymbol{P}} / \gamma\right)^{2} / 3, \boldsymbol{M}_{\text {sol }}=2 \boldsymbol{m}_{\text {ex }}\left[1+32\left(4 \pi \alpha_{\mathbf{P}}\right)^{2} / 3\right]
$$

From the above parameter values in Eq.(18), we obtain $\gamma=0.08$. Utilizing these values, the $E_{B D} / J$ versus $4 \pi \alpha$ relations in Eq.(18) are plotted in figure 2.

However, $\boldsymbol{E}_{B \boldsymbol{B}} / \boldsymbol{J}=\left(4 \pi \alpha_{\boldsymbol{D}} / \gamma\right)^{2} / 3$ for the Davydov model, where

$$
\left.\boldsymbol{M}_{\text {sol }}^{\prime}=\boldsymbol{m}_{e x}\left[1+2\left(4 \pi \alpha_{P} / \gamma\right)^{2} / 3\right] \text { and } 4 \pi \alpha_{D}=\chi_{1}^{2} / 2 \boldsymbol{w} \hbar \omega_{D}\right) \text {, then the } E_{B D} J \text { versus } 4 \pi \alpha_{D}
$$

relation is also plotted in figure 2. From this figure we see that the difference of soliton binding energies between two models becomes larger with increasing $4 \pi \alpha$

Meanwhile, we see clearly from Eqs.(12)-(15) and (16) that the localized feature of the new soliton is enhanced due to increases of the nonlinear interaction and its binding energy resulting from the increases of exciton-phonon interaction in the improves model. Thus, the stability of the new soliton against quantum and thermal fluctuations is enhanced considerately. In fact, the nonlinear interaction energy forming new siliton in the new model is

$\mathrm{G}_{\mathrm{p}}=8\left(\chi_{1}+\chi_{2}\right)^{2} /\left(1-s^{2}\right) w=3.8 \times 10^{-21} \mathrm{~J}$, and it is larger than the linear dispersion energy, $\mathrm{J}=1.55 \times 10^{-22} \mathrm{~J}$, i.e., the nonlinear interaction in this model is so large that it can actually cancel or suppress the dispersion effect in the equation of motion ,thus the new soliton is stable in such a case according to the soliton theory [5-14,137] (Figure 2).

On the other hand,the nonlinear interaction energy in the Davydov model is $\mathrm{G}_{\mathrm{D}}=4 \chi_{1}^{2} /\left(1-s^{2}\right) w=1.18 \times 10^{-21} \mathrm{~J}$, and it is about three to four times smaller than $G_{p}$.

Therefore, the stability of the Davydov soliton is weaker as compared with the new soliton. Moreover, the binding energy of the new soliton in the improved model is $E_{B P}=$ $(4.16-4.3) \times 10^{-21} \mathrm{~J}$ in Eq.(15), which is somewhat larger than the thermal perturbation energy, $K_{B} T=4.13 \times 10^{-21} \mathrm{~J}$, at $300 \mathrm{~K}$ and about four times larger than the Debye energy, $K_{B} \Theta=\hbar \omega_{D}=1.2 \times 10^{-21} J$ (there $\omega_{D}$ is the Debye frequency).This shows that transition of the new soliton to a delocalized state by the heat energy can be suppressed by the large energy difference between the initial (solitonic) state and final (delocalized) state, which is very difficult to compensate for with the energy of the absorbed phonon. Thus, the new soliton is robust against quantum and thermal fluctuations, therefore it has a large lifetime and good thermal stability in the region of biological temperature .In

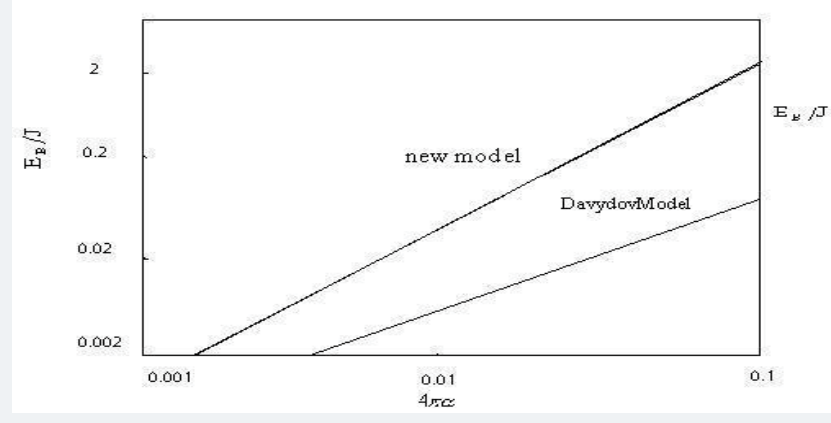

Figure 2: The binding energy $\left(E_{B}\right)$ of the solitons in units of dipole-dipole interaction energy $(J)$ vs the coupled constant, $4 \pi \alpha$, relation in Pang's model and the Dvydovy's model. 
practice, according to Schweitzer et al.'s studies (i.e the lifetime of the soliton increases as $\mu_{p}$ and $T_{0}=\hbar v_{0} \mu_{p} / K_{B} \pi$ increase at a given temperature)[67-68] and from the above results obtained we infer that the lifetime of the new soliton will increase considerably relative to that of the Davydov soliton due to the increae of $\mu_{p}$ and $T_{0}$ because the latter are about three times larger than that of the Davydov model.

On the other hand the binding energy of the Davydov soliton, $\mathrm{E}_{\mathrm{BD}}=\chi_{1}^{4} / 3 \mathrm{w}^{2} \mathrm{~J}=0.188 \times 10^{-21} \mathrm{~J}$, is about 23 times smaller than that of the new soliton, about 22 times smaller than $K_{B} T$, and about 6 times smaller than $K_{B} \Theta$, respectively. Therefore, the Davydov soliton is easily destructed by the thermal perturbation energy and quantum transition effects. Thus we can naturally obtain that the Davdov soliton has only a very small lifetime, and is unstable at the biological temperature $300 \mathrm{~K}$. This conclusion is consistent at a qualitative level with the result s of Wang et al. [64,65] and Cottingham et al. $[66,67]$.

In the above investigation of the influences of quantum and thermal effects on soliton state, which are expected to cause the soliton to decay into delocalized states, we postulate that the model Hamiltonian and the wavefunction in the new improved model together give a complete and realistic picture of the interaction properties and allowed states of the protein molecules. The additional interaction term in the Hamiltonian gives a one-to-one correspondence of interactions in the new model. The new wavefunction is a reasonable choice for the protein molecules because it not only can exhibit the coherent features of collective excitations arising from the nonlinear interaction between the excitons and phonons, but also retain the conservation of number of particles and fulfil the fact that the energy released by the ATP hydrolysis can only excite two quanta. In such a case, using a standard calculating method $[2,26]$ and widely accepted parameters in Eq.(17) we can calculate the region encompassed of the excitation or the linear extent of the new soliton, $\Delta X=2 \pi r_{0} / \mu_{p}$, which is greater than the lattice constant $r_{0}$ i.e., $\Delta X>r_{0}$. Meanwhile, we can explicitly calculate the amplitude squared of the new soliton using Eq.(13) in its rest frame, which is $|\phi(x)|^{2}=\frac{\mu_{p}}{2} \sec h^{2}\left(\frac{\mu_{p} x}{r_{0}}\right)$. Thus the probability to find the new soliton outside a range of width $r_{0}$ is about 0.10 . This means that the new soliton is very well localized in this condition. Meanwhile, this number can be compatible with the continuous approximation since the quasi-coherent soliton can spread over more than one lattice spacing in the system in such a case. Thus, this proves that assuming of the continuous approximation used in the calculation is valid because the soliton widths is large than the order of the lattice spacing, then the soliton stability is improved.

Finally we calculate the values of the main parameters in the new model by the above values. These values and the corresponding values in the Davydov model are simultaneously listed in table 1 . From table 1 we can see clearly that the new model produces considerable changes in the properties of the soliton, for example, large increase of the nonlinear interaction, binding energy and amplitude of the new soliton, and decrease of its width as compared to those of the Davydov soliton. This shows that the soliton in the new model is more localized and more robust against quantum and thermal fluctuations, thus its stability is enhanced [5-14, 104-125], which implies an increase in lifetime for the new soliton. From Eq.(16) we also find that the effect of the two-quanta nature is larger than that of the added interaction. We thus can refer to the new soliton as quasi-coherent state, which is novel and correct wave function.

Table 1: Comparison of parameters used in the Davydov model and Pang's model.

\begin{tabular}{|c|c|c|c|c|c|}
\hline $\begin{array}{c}\text { Parameters } \\
\text { Models }\end{array}$ & $\boldsymbol{\mu}$ & $\mathbf{G}\left(\times \mathbf{1 0}^{-21} \mathbf{J}\right)$ & $\begin{array}{c}\text { Amplitude of soliton } \\
\mathbf{A}^{\prime}\end{array}$ & Width of soliton $\mathbf{\Delta \times}\left(\times \mathbf{1 0}^{-10} \mathbf{m}\right)$ & $\begin{array}{c}\text { Binding energy of soliton } \\
\mathbf{E}_{\mathbf{B}}\left(\times 10^{-21} \mathbf{J}\right)\end{array}$ \\
\hline $\begin{array}{c}\text { Pang's } \\
\text { Model }\end{array}$ & 5.94 & 3.8 & 1.72 & 4.95 & -4.3 \\
\hline $\begin{array}{c}\text { Davydov } \\
\text { model }\end{array}$ & 1.90 & 1.18 & 0.974 & 14.88 & -0.188 \\
\hline
\end{tabular}




\section{The necessity verifying the validity of these theoretical models}

We exhibit the properties, successful and problems of different models in the above investigation, which but leaves behind many questions that are worth to study continuously. Actually, the above results were obtained analytically, based on many hypotheses, in which the protein molecules, which were used by the researchers, was regarded as a periodically and uniformly infinite-long chains composed of amino acid residues with same weight. Obviously, the proteins are not completely conformable with the biological protein molecules in the living systems. As it is known, the biological proteins are an finite -long structure, which are composed of several hundreds or thousands amino acid residues with different molecular weights between $75 \mathrm{~m}_{\mathrm{p}}$ (glycine) and $204 \mathrm{~m}_{\mathrm{p}}$ (tryptophan), which correspond to variations in mass between $0.67 \bar{M}<M<1.80 \bar{M}$, where $\bar{M}=114 \mathrm{mp}$ is the average mass of an amino acid residue and $m_{p}$ is the proton mass. And the biological proteins adhere all some side groups, which will affect the structure and dynamic features of protein molecules. This means that there are a structure nonuniformity and disorder in biological-protein molecules. These structural nonuniformities result necessarily in the fluctuations of the spring constant, the dipole-dipole interaction, the exciton-phonon coupling constant and the diagonal disorder in the equations of motion. Thus, the states of the solitons obtained from the theoretical models, which are established based on uniform proteins, will be changed under influence of these structure disorders in the biological protein molecules. Otherwise, in the above investigation all physical parameters of the protein molecules were represented by their average values, and some approximation methods, such as long- wavelength approximation, continuum approximation, or long-time approximation, were also used in concrete calculations, which cannot be evidently used in the biological proteins. Careri et al. [19,138-143], demonstrated that even relatively small amounts of disorder in an amorphous film of acetanilide (ACN), a protein-like crystal, is enough to destroy the spectral signature of a "soliton". Therefore, we have the reasons to doubt the real existence of the solitons and him correctness of the above theory of bio-energy transport in protein molecules.

At the same time, the biological proteins work always at physiological temperature and biological solution containing water molecules and other ions. The thermal perturbation and damping of medium arising from the temperature will also change the states and lifetimes of the solitons, which are the carrier of the bio-energy transport. These water molecules and other ions existed in the solution serve as some imported impurities to influence the dynamic features of the protein molecules. On the other hand, for the $\alpha$-helix protein molecules, which are constructed by three channels, there are also chain-chain interaction among the three channels, which carry also a dispersive effect and further influence the states of the solitons in different models.

In such a case, we have the reason to doubt the validity of the above theories of bio-energy transport. This means that we must verify whether these models are appropriate to biological proteins? Whether can the models represent the real features of biological protein molecules? Namely, it is quite necessary to verify the validity of these theories in the biological protein molecules and to check the states and properties of the bio-solitons under the influences of these structure nonuniformlity, chain-chain interaction and temperature and damping of the medium in the protein molecules.

However, what are methods which can be used to verify and check the correctness of the theories of bio-energy transport? The numerical simulation is just a best method studying and solving these problems. In fact, the numerical simulation can work at the protein molecules with a finite length, it abandons the various approximations used in analytic calculations, and can include the effects of structure nonuniformity, imported impurities and side groups of proteins and influences of temperature and damping of medium on the bio-energy transport. Therefore, the numerical calculation can be used 
to study the properties of the solitons or bio-energy transport in the really biologicalprotein molecules. In particle, a lot of scientists and researchers, containing Davydov, Scott et al., Wang X et al, Forner and Pang XF, used the different numerical-simulation methods to investigate extensively the properties of bio-energy transport in single chain protein and t $\alpha$-helix protein molecules with three channels, respectively, where a lot of researched results, which are enough to assess and check the correctness of theory of bio-energy transport in the biological proteins, involving Davydov's and Pang's models, were obtained. These numerical- simulation techniques contain the average Hamiltonian way of thermal perturbation, fourth-order Runge-Kutta method, Monte Carlo method, quantum perturbed way and thermodynamic and statistical method, and so on. We here review widely and in-depth the progress of different numerical simulations and give their results, which can be used to assess and verify the validity and availability of the theories of bio-energy transport mentioned above. For this purpose we here stated and discussed following four problems.

\section{Properties of energy transport in uniform protein molecules}

\section{Determinations of value of basic parameters in energy transport theory}

As it is known, the properties of bio-energy transport are closely related to the structure features of $\alpha$-helix protein molecules. If the parameters of structure of proteins are different, then the property of the energy transport will also be changed. Therefore, correct determination of the values of these parameters contained in these theories is very important for verifying the validity of the theory of bio-energy transport. As it is known, there are five basic parameters, the mass of amino acid residue, $M$, the spring constant, w, dipole-dipole interaction constant, J, exciton-phonon coupling constant $\chi_{1}$ and ground state energy $\varepsilon_{0}$ in Davydov model, but another coupling constant $\chi_{2}$ to be included in Pang's model for the protein molecules, of single chain. For these parameters in the protein molecules, the widely accepted and available values are given in Eq.(17), where $m_{p}=1.67 \times 10^{-27} \mathrm{~kg}$ is proton masse, $r_{0}$ is lattice constant or distance between two amino acids. For $\alpha$-helix proteins there is again a chain-chain interaction coefficient $\mathrm{L}$, which is $\mathrm{L}=1.5 \mathrm{meV}$, among the three chains. The above values are in essence their average values, such as $M$ here is an average value of 20 different amino acids.

In these parameters the determination of exciton-phonon coupling constants plays a key part in the correctness of the bio-energy transport theory, meanwhile, it is also quite difficult to determine. As it is known, $\chi_{1}$ and $\chi_{2}$ represent the changes of ground state energy and dipole-dipole interactional energy when the amino acid residue displaces one unit distance, respectively. Experimental value of $\chi_{1}$ is in the range of $35-62 \mathrm{PN}$, its concrete value was obtained numerical calculation. A primary motivation for early numerical simulation of Davydov theory [5-14] was just to determine the magnitude of the exciton-phonon coupling coefficient $\chi_{1}$. Ones thought that $\chi_{1}$ should be enough large to support soliton transport of the bio-energy in the $\alpha$-helical protein. To proceed seriously, the following assumptions are made. (1) Initial amide-I energy was localized in a single turn of the helix, A typical initial condition to launch a symmetrical soliton is $\varphi_{11}^{0}=\varphi_{12}^{0}=\varphi_{13}^{0}=\sqrt{2 / 3}$ and $\varphi_{n n}^{0}=0$ for $\mathrm{n}>1$. This means that two amide-I quanta were put into the first turn at one end of the helix at time $t=0$.

(2) All important dipole-dipole interactions were calculated. Then other larger interactions was considered and eight longer range interaction terms were also involved, except for the neighboring interactions J and A numerical code that embodied these two assumptions is developed in the early 1980s [15,16, 68,144]. This code denoted a helix of 200 turns, which is about the length of the alpha-helix in myosin, with free end boundary conditions. With the above initial conditions we can obtain that a threshold value for a soliton to be formed is the exciton-phonon coupling coefficient to equal to $\chi_{1} \geq 50 \mathrm{pN}$. 
If the two quanta of the above initial conditions were considered by the wave function proposed by Kerr et al. [145], $|\Psi\rangle=(1 / \sqrt{Q})\left(\sum_{n} \varphi_{n}(t) B_{n}^{+}\right)^{Q}|0\rangle_{\text {ex }}$, then the threshold value will be increased.

However, the exciton-phonon coupling coefficient for a really biological alpha-helix protein molecules should be large enough to ensure the soliton formation, which but have been challenged by Mechtly and Shaw [70]. They constructed a wave function for the Davydov Hamiltonian, which is more accurate than Davydov's product wave function because it is an eigenstate of the translation operator over the range of $0 \leq$ $4 \pi \alpha \leq 1$, where $4 \pi \alpha$ is a coupling parameter which was defined in Ref.[15-20]. Then its dynamic equations differ from the Davydov's. Since numerical simulation of the dynamic equations with the above initial conditions indicates the soliton is formed only for $4 \pi \alpha>1$. Thus Machtly et al. [70], obtained that the previous threshold value of $\chi_{1}$ for soliton formation is underestimated by a factor of at least four. In this case the effective mass of the soliton rises quadratically, then Heisenberg's uncertainty relation $\Delta x \Delta v \geq \hbar / 2 M_{\text {eff }}$ becomes closer to that of a classical object. So, this result is doubted.

Rhodes and Nichclls [146] studied also this problem, in which he introduce a wave function that is based on a time dependent unitary transformation. The numerical calculations shows delocalization of the wave packet as is required by the above uncertainty relation.

From the above investigations we can conclude the following results.

(A) Numerical studies of Davydov model shows a threshold level of the excitonphonon coupling coefficient $\chi_{1}$ above which amide-I energy, that is initially localized on a single turn of the alpha helix, is converted into a soliton. The threshold level is $\chi_{1}$ $\geq 50 \mathrm{pN}$, which agrees with independent experimental measurement of $\chi_{1}>35 \mathrm{pN}$ to $62 p N$

(B). Numerical simulation of improved Davydov's wave functions $[70,146]$ that indicates the larger threshold values of $\chi_{1}$ ware misleading. The larger threshold values are an artifact of the wave packet feature of the improved wave function. Therefore it not credible.

The results of different methods of numerical simulation for the solution of dynamic equations in Davydov model in uniform protein chains

Hyman, McLaughlin, and Scott [68] studied first the properties of the bio-energy transport in alpha-helix protein molecules with three channels in Davydov model [5-14] using numerical simulation mother with the help of the computer at the Los Alamos Scientific Laboratory in 1979. In this case the interaction among the channels is included in the Hamiltonian of the protein molecules. From the average Hamiltonian they gave the discrete forms of dynamic equation of the exciton and phonon in the biological protein with three channels, which are denoted by

$$
\begin{aligned}
& i \hbar \dot{\varphi}_{n \alpha}=\left[\varepsilon_{0}+W+\chi_{1}\left(u_{n+1, \alpha}-u_{n-1, \alpha}\right)\right] \varphi_{n \alpha}+J\left(\varphi_{n+1, \alpha}-\varphi_{n-1, \alpha}\right)+L\left(\varphi_{n, \alpha+1}-\varphi_{n, \alpha-1}\right) \\
& M \ddot{u}_{n \alpha}=w\left(u_{n+1, \alpha}-2 u_{n, \alpha}+u_{n-1, \alpha}\right)+\chi_{1}\left(\left|\varphi_{n+1, \alpha}\right|^{2}-\left|\varphi_{n-1, \alpha}\right|^{2}\right), \alpha=1,2,3 .
\end{aligned}
$$$$
\text { where } W=\frac{1}{2} \sum_{n}\left[M\left(\frac{\partial u_{n, \alpha}}{\partial t}\right)^{2}+w\left(u_{n+1, \alpha}-u_{n, \alpha}\right)^{2}\right] \text {. Equations (19) and (20) were }
$$

integrated numerically by them for studying the excited states of three chains of helical protein molecules. Each chain contained 200 peptide groups. The molecule was characterized by the following quantities: $\varepsilon_{0}=0.205 \mathrm{eV} ; \mathrm{M}=70 \mathrm{~m}_{\mathrm{P},} v_{0=} 10^{4} \mathrm{~cm} / \mathrm{s}, J=1.55$ $\times 10^{-22} \mathrm{~J}, L=2.46 \times \times 10^{-22} \mathrm{~J}$, 
$w=76 \mathrm{~N} / \mathrm{m}, \chi_{1=}(5-7) \times 10^{-11} \mathrm{~N}$. The initial conditions at $t=0$ were taken as $\phi_{n a}=1$ for $\mathrm{n}=1$ or $=0$ for $n \neq 1, u_{n x}=0$. The calculations were performed for different values of the coupling parameter $\chi_{1}$ of intrapeptide Amide I excitations with displacements of their equilibrium positions. It was shown that for the above initial conditions distinct solitons are formed and propagate in the proteins with $\chi 1 \geq 3 \times 10^{-11} \mathrm{~N}$. Solitons with $\chi 1$ close to the critical value propagate with velocity $\sim 1.26 \cdot 10^{3} \mathrm{~m} / \mathrm{s}$. Therefore, the distance 17000 nanometers, corresponding to the length of the alpha-helical myosine molecule in muscle fibers $[5,6]$, could be traversed by solitons (where the friction forces of medium is neglected) within 130 PS.

Hyman et al. [68], found that the conclusion obtained from the numerical studies of Davydov's dynamic model in the alpha-helical proteins confirm his prediction of the formation of solitons; these solitons are robust, localized, and a dynamic entity that coupled amide-I vibrations to longitudinal sound waves, thus they may provide an efficient mechanism of energy transport in biological systems. Both the numerical studies and analytical computations show a threshold level of nonlinearity below which the solitons will not be formed. A rough estimate indicates that this nonlinearity has the required order of magnitude.

Hyman et al's result is interesting in that the process of formation of solitons from a definite initial state is investigated and the role of the discreteness of the chain, which, apparently reduces to the fact that solitons are formed in the chain only with supercritical values of the coupling parameter $\chi 1$, determining the nonlinearity of the system, is clarified.

In 1979, Eilbeck made a computer film that demonstrates the propagation of an internal vibrational excitation of an edge group along a peptide groups (PG) chain. This film clearly shows that for an above threshold value of the coupling parameter $\chi 1$ between the vibrational excitation and displacements of PG along the molecular chains, the excitation propagates in the form of a soliton, i.e., in the form of a local pulse, whose shape and width remain constant during the motion. Eilbeck's film is important for two reasons: first, it confirms the previous calculations performed at the Los Alamos Laboratory and, second, it clearly demonstrates the stability of solitons relative to interactions with sound waves. The sound wave was excited together with the soliton. Moving faster than the soliton, it reflected several times from the ends of the chain passing through the soliton and not causing any changes in it.

Eilbeck's film and the numerical calculations in Ref. 68 show that the soliton forms at the very beginning of the peptide chain. Therefore, solitons can arise within comparatively short sections of alpha-helical proteins.

Scott $[15-18,68]$ modified further Davydov's dynamic-equations for the alpha helix soliton to include ten additional dipole-dipole interactions and represent helical symmetry, Thus the equations (19)-(20) were replaced by

$$
\begin{aligned}
& i \hbar \dot{\varphi}_{n \alpha}=\left[\varepsilon_{0}+W+\chi_{1}\left(u_{n+1, \alpha}-u_{n-1, \alpha}\right)\right] \varphi_{n \alpha}+J\left(\varphi_{n+1, \alpha}-\varphi_{n-1, \alpha}\right)+L\left(\varphi_{n, \alpha+1}-\varphi_{n, \alpha-1}\right)+ \\
& \left.\left.\chi_{2}^{\prime}\left[\left(u_{n+1, \alpha}-u_{n, \alpha}\right)\right] \varphi_{n=1, \alpha}+\left(u_{n, \alpha}-u_{n-1, \alpha}\right)\right] \varphi_{n-1 \alpha}\right]+N F_{N}+P F_{P}+Q F_{Q}+R F_{R}+S F_{S}+ \\
& T F_{T}+U F_{U}+V F_{V}+W F_{W}+X F_{X}+Z F_{Z} \\
& \quad \ddot{\ddot{u}}_{n \alpha}=w\left(u_{n+1, \alpha}-2 u_{n, \alpha}+u_{n-1, \alpha}\right)+\chi_{1}\left(\left|\varphi_{n+1, \alpha}\right|^{2}-\left|\varphi_{n-1, \alpha}\right|^{2}\right)+ \\
& \chi_{2}^{\prime}\left[\varphi_{n \alpha}^{*}\left(\varphi_{n+1, \alpha}-\varphi_{n-1, \alpha}\right)+\left(\varphi_{n+1, \alpha}^{*}-\varphi_{n-1, \alpha}^{*}\right) \varphi_{n \alpha}\right]
\end{aligned}
$$

where the J,L, N,F,Q,R,S,T, S, T,U,V,W, X, Z are the dipole-dipole interaction energies between pairs of amide-I bonds,which are 0.143, 0,231, 0.073, 0.03, 0.019, 0.012, 
$0.0089,0.0073,0.0037,0.0030,0.0022,0.0017$, respectively. The term " $\mathrm{NF}_{\mathrm{N}}$ " is shorthand for the dipole-dipole interaction between laterally adjacent amide-I bonds, which can be denoted as

$$
i \hbar \dot{\varphi}_{n \alpha}=\mathbf{N}\left(\varphi_{n, \alpha+1}+\varphi_{n, \alpha-1}\right)+\ldots, n=1,2,3 \ldots ., \alpha=1,2,3 .
$$

Scott et al. $[15-18,68]$ found the solutions of the above equations by numerical calculation method, and obtained that there is a "window" for soliton formation when $\chi_{1}$ lies in the range of $0.35 \times 10^{-10} N<\chi 1 \leq 0.6 \times 10^{-10} N$, i.e., the exciton-phonon coupling parameter in alpha-helix protein molecules is large enough to transport the formation of Davydov solitons under normal physiological condition. Meanwhile, they also gained the following conclusions from the studies:

(1) It should be possible to create Davydov solitons by direct stimulation with infrared radiation of $6.06 \mu \mathrm{m}$ (corresponding to an amide-I absorption at $1650 \mathrm{~cm}^{-1}$ ). This is clear because in the numerical study only bond energy was introduced as an initial condition.

(2) Davydov solitons should display a rather sharp internal resonance (related to exchange of energy between the three longitudinal spines of the alpha helix) with a period of about 2 psec or a free-space wavelength of about $600 \mu \mathrm{m}$.

(3) A piece of alpha-helix with $\mathrm{n}$ unit cells (i.e., $3 \mathrm{n}$ peptide units) may show a broad resonance ("Q"='1) with a period of about $0.54 \mathrm{n}$ psec. Since the molecular weight $\left(\mathrm{W}_{100}\right)$ of a strand of helix is about $114 \mathrm{n}$, this resonance should appear at a free-space wavelength of $1.42 W_{100} \times 10^{4} \mathrm{~cm}$. For this resonance to appear at $3 \mathrm{~cm}$ ("X-band" in RADAR jargon) the molecular wavelength would be about $W_{100}=21000$. For the resonance to appear at $30 \mathrm{~cm}(1 \mathrm{GHz}) W_{100}=210000$.

(4) Mechanical effects induced directly by the soliton are limited to the kinetic part of the total energy. For the example studied here this is less than $0.02 \%(0.0002)$ of the total energy.

Mechtly et al. [70], develop a theory of exciton evolution on a zero-temperature Davydov lattice which is free of certain deficiencies found in the standard Davydov theory. The approach makes use of a time-dependent unitary transformation on a Davydov Hamiltonian parametrized by a dimensionless lattice constant and a dimensionless exciton-phonon coupling constant $\alpha$. The transformation generator is expanded in a normal-ordered series of multiphonon operators with expansion coefficients chosen to eliminate various terms in the transformed Schrodinger equation. At the one-phonon level, they obtained equations of motion which differ from those of Davydov. In the small-polaron transportless limit (infinite a) the equations are exact. In the large-polaron continuum limit (vanishing a) the equations become field equations whose stationary solutions are those of Gross's interpolation theory. To use numerical simulation method for a one-spine model of an $\alpha$-helix $(a=2.7)$ they found that the soliton (a moving dispersionless excitation) formation during evolution from a localized initial state above a threshold value of $4 \pi \alpha>1$, which is an order of magnitude higher than the threshold found in standard Davydov theory. This lower threshold for the Davydo theory is due to its inadequate treatment of the exciton- phonon interaction in weak coupling.

Since the value of the coupling-constant threshold obtained within Mechtly et al's one-phonon approximation exceeds the range of values for the $\alpha$-helix suggested by MacNeil et al. [70], it is tempting to conclude that an $\alpha$-helix can not support solitons at $\mathrm{T}=0$. Thus it would be straightforward to extend the present analysis to test this conclusion on more realistic models of an $\alpha$-helix. As a check on the validity of the onephonon approximation itself, an extension of the present theory to the two-phonon level is done further. 
Förner [50] studied the changes of dynamical properties of Davydov soliton in an uniform protein molecular chain using a fourth-order Runge-Kutta method [147,148]. In this method the dynamic equations of simulation in Davydov model, which can be obtained from Eq.(1)-(2) , are as follows

$$
\begin{aligned}
& i \hbar \dot{\varphi}_{n}=\left[\varepsilon_{0}+\chi_{1}\left(u_{n+1}-u_{n-1}\right)\right] \varphi_{n}-\left(J_{n} \varphi_{n+1}+J_{n-1} \varphi_{n-1}\right) \\
& \left.P_{n}=w_{n+1}\left(u_{n+1}-u_{n}\right)-w_{n}\left(u_{n}-u_{n-1, \alpha}\right)+\chi_{1(n+1)}\left|\varphi_{n+1}\right|^{2}-\chi_{1(n)}\left|\varphi_{n}\right|^{2}\right) \\
& \text { where } P_{n}=M \dot{u}_{n}, \varphi_{n}^{\prime}=\varphi_{n} \exp \left[-i \varepsilon_{0} t / \hbar\right]
\end{aligned}
$$

In this method, he used a time step size of $0.01 \mathrm{ps}$, the total energy was conserved to $3 \mu \mathrm{eV}(0.015 \%)$; a possible imaginary part of the energy, which can occur due to numerical inaccuracies was zero to an accuracy of $0.002 \mathrm{feV}$, the norm is conserved up to $0.4 \mathrm{pp}$ (parts per million). Otherwise, the chain ends are fixed and an initial excitation with one quantum is put at the site $\mathrm{N}-1$, which $\mathrm{N}$ is the number of units chosen to be $\mathrm{N}=200$ in the calculation. For the lattice $u_{n}(0)=P_{n}(0)=0$ were used.

This simulation result shows that there is a window of the characteristic parameters of $\chi_{1}$ and $\mathrm{w}$ for the occurrence of Davydov soliton, which are $60 \mathrm{PN}<\chi_{1}<140 \mathrm{PN}$ and $30 \mathrm{~N} / \mathrm{m},<\mathrm{w}<90 \mathrm{~N} / \mathrm{m}$. Meanwhile, the motions of Davydov soliton with changing the time and position are shown in figure 3 , where we see clearly that the soliton is dispersive at $\chi_{1}=20 \mathrm{PN}$, is gradually formed with increasing $\chi_{1}$, but the soliton is still somewhat dispersive at $\chi_{1}=60 \mathrm{PN}$ and is stable at $\chi_{1}=100 \mathrm{PN}$, which is larger than the widely accepted value of $62 \mathrm{PN}$.

The above results of numerical simulations in an uniform protein molecular chain exhibit clearly that the Davydov soliton can be form, but is not stable for small $\chi_{1}$, which is lower than the widely accepted values of physical parameters shown in Eq.(1). This conclusion is consistent from Lomdahl et al's result of numerical simulation [62], which indicates that the solitons are nucleated from random initial conditions, but could be dispersed in motion along the chain as shown in figure 3.
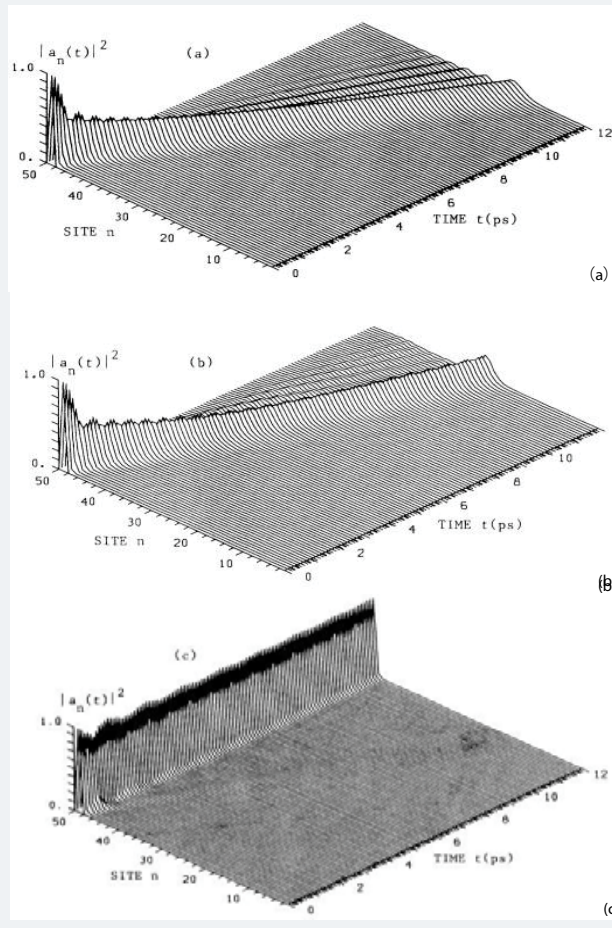

Figure 3: The changes of moved state of Davydov soliton with $\left|D_{2}\right\rangle$ ansatz in an uniform protein molecular chain obtained by Förner [50] 


\section{The properties of numerical simulation of energy transport in Pang's model}

The results in a signal protein chain: We first adopt the discrete dynamic equations (6)-(7) in Pang's model to simulate numerically the dynamic properties of the soliton in single chain proteins. We now replace the $\phi_{n}(t)$ and ${ }_{\beta n}(t)$ in Eqs. (6)-(7) by $a_{n}(t)=\mathrm{a}_{\mathrm{n}}(\mathrm{t}) \mathrm{r}_{\mathrm{n}}+\mathrm{ia}(\mathrm{t}) \mathrm{i}_{\mathrm{n}}$ and $q_{n}(t)$, respectively, where $a r_{n}$ and $a i_{n}$ are real and imaginary parts of $\mathrm{a}_{\mathrm{n}}(\mathrm{t})$, and use the transformation: $\mathrm{a}_{\mathrm{n}}(\mathrm{t}) \rightarrow \alpha_{n} \exp [\operatorname{Ir}(t) t / h$ to eliminate the term $\mathrm{R}(\mathrm{t}) \mathrm{a}_{\mathrm{n}}(\mathrm{t})$ in Eq. (11). Thus equations (11) and (12) can then rewritten as[104- 125]

$$
\begin{aligned}
& \hbar \dot{a} r_{n}=-J\left(a i_{n+1}+a i_{n-1}\right)+\chi_{1}\left(q_{n+1}-q_{n-1}\right) a i_{n}+\chi_{2}\left(q_{n+1}-q_{n}\right)\left(a i_{n+1}+a i_{n-1}\right) \\
& \hbar \dot{a} r_{n}=-J\left(a i_{n+1}+a i_{n-1}\right)+\chi_{1}\left(q_{n+1}-q_{n-1}\right) a i_{n}+\chi_{2}\left(q_{n+1}-q_{n}\right)\left(a i_{n+1}+a i_{n-1}\right) \\
& \dot{y}_{n}=w\left(q_{n+1}-2 q_{n}+q_{n-1}\right)+2 \chi_{1}\left(a r_{n+1}^{2}+a i_{n+1}^{2}-a r_{n-1}^{2}-a i_{n-1}^{2}\right)+4 \chi_{2}\left[a r_{n}\left(a r_{n+1}-a r_{n-1}\right)+a i_{n}\left(a i_{n+1}-a i_{n-1}\right)\right]
\end{aligned}
$$

where $\dot{q}_{n}=y_{n} / M,\left|a_{n}\right|^{2}=\left|a r_{n}\right|^{2}+\left|a i_{n}\right|^{2}$. Equations (25)-(27) are dynamic equations, which can determine the states and behaviors of the soliton. We will obtain their solutions numerically using the fourth-order Runge-Kutta method [147-148]. Obviously, there are four equations for one peptide group. Therefore, for protein molecules of single chain constructed by $\mathrm{N}$ amino acids we have to solve a system of $4 \mathrm{~N}$ associated equations. To use the fourth-order Runge-Kutta method [147-148] we need first to discretize the equations, in which, the time be discretized and denoted by $j$, the step size of the space variable is denoted by $h$. In the numerical simulation, Pang et al., used eV for energy, $\stackrel{\circ}{\mathrm{A}}$ for length, and ps for time [104-125]. The followings are details of the numerical simulation: a time step size of $0.0195 \mathrm{ps}$, the total energy $\mathrm{E}=<\Phi(\mathrm{t})|\mathrm{H}| \Phi(\mathrm{t})>$ was conserved to $0.0012 \%$ ( a possible imaginary part of the energy can be developed during the simulation due to numerical inaccuracies which was kept to below $0.001 \mathrm{feV}$ ), the norm is conserved up to $0.3 \mathrm{pp}$ (parts per million). A fixed chain with $\mathrm{N}$ units and an initial excitation in the forms of $\mathrm{a}_{\mathrm{n}}(\mathrm{o})=\operatorname{Asech}\left[\left(\mathrm{n}-\mathrm{n}_{0}\right)\left(\chi_{2}+\chi_{1}\right)^{2} / 4 \mathrm{Jw}\right]$ at the site $\mathrm{n}$ were used in this calculation, where $\mathrm{A}$ is the normalization constant,. For the lattice, the initial conditions of $q_{n}(0)=\pi_{n}(0)=0$ was applied. Pang et al., chose $N=50$ in this simulations. Meanwhile, the simulation was performed using a data parallel

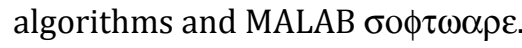

Utilizing the above average values for the parameter, which are now denoted by $\bar{M}, \bar{w}, \overline{\varepsilon_{0}}, \bar{J}, \quad \chi_{1}$ and $\chi_{2}$, we calculate numerically the solution of Eqs.(25)-(27) by using the fourth-order Runge-Kutta method in uniform and periodic proteins, where $\left|a_{n}\right|^{2}$ is the number density of the soliton occurring at the nth amino acid residue. Thus, we can plot the state of motion of the soliton in time-place. The result is shown in figure 4. This figure shows that the amplitude of the solution can retain constancy. In figures 5,6 we show the propagation behavior of the solution for a long time period of 300Ps, and the collision property of two solitons, respectively. From the figures we see that the solution is very stable while in motion for a long time period. Thus, Eqs. (25)- (27) have exactly a soliton solution in uniform and periodic proteins, which coincide with the above analytic results.

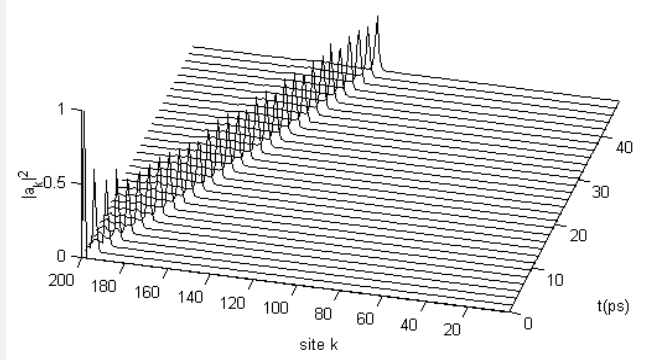

Figure 4: Soliton solution of Eqs. (25) - (27) in uniform chains. 


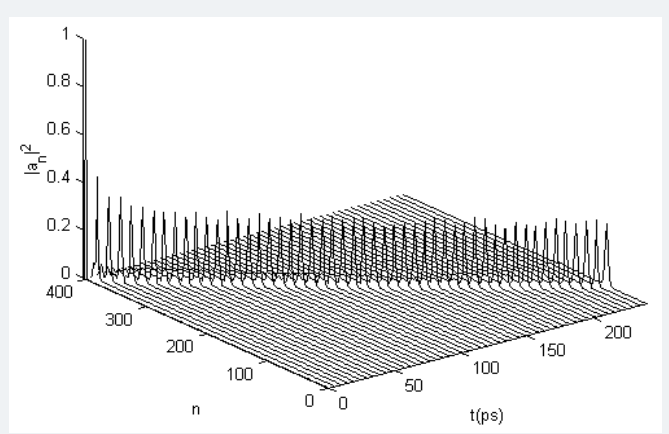

Figure 5: State of Pang's soliton in the cases of large time of 300ps and long distances of 400.

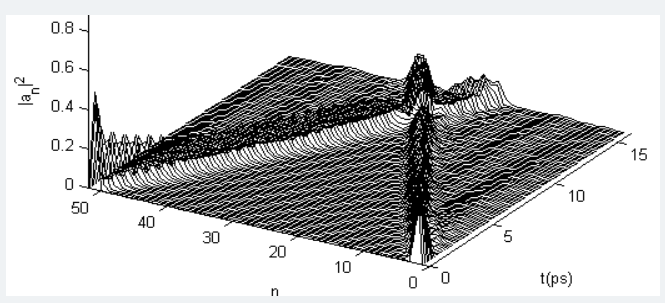

Figure 6: The collision behavior of two Pang's solitons for Eqs. (25) - (27).

\section{The results in $\alpha$-helix protein molecules with three channels}

In the meanwhile, we numerically simulated also the properties of the solutions of the dynamic equations for in the $\alpha$-helix protein molecules with three channels. In this case, the Hamiltonian and the wave function in Eqs. (6) and (7) in Pang's model[104-125] are now, respectively, replaced by

$$
\begin{gathered}
H=\sum_{n}\left[\varepsilon_{0} B_{n \alpha}^{+} B_{n+1 \alpha}-J\left(B_{n \alpha}^{+} B_{n+1 \alpha}+B_{n \alpha} B_{n+1 \alpha}^{+}\right)\right]+\sum_{n}\left[\frac{P_{n \alpha}^{2}}{2 M}+\frac{1}{2} w\left(q_{n \alpha}-q_{n-1 \alpha}\right)^{2}\right]+\sum_{n}\left[\chi_{1}\left(q_{n+1 \alpha}-q_{n-1 \alpha}\right) \times\right. \\
\left.B_{n \alpha}^{+} B_{n \alpha}+\chi_{2}\left(q_{n+1 \alpha}-q_{n-1 \alpha}\right)\left(B_{n+1 \alpha}^{+} B_{n \alpha}+B_{n \alpha}^{+} B_{n+1 \alpha}\right)+L\left(B_{n \alpha}^{+} B_{n+1 \alpha}+B_{n \alpha}^{+} B_{n-1 \alpha}\right)\right], \alpha=1,2,3 . \\
|\Phi(t)\rangle=|a(t)\rangle|\beta(t)\rangle=\frac{1}{\lambda}\left[1+\sum_{n \alpha} a_{n \alpha}(t) B_{n \alpha}^{+}+\frac{1}{2 !}\left(\sum_{n \alpha} a_{n \alpha}(t) B_{n \alpha}^{+}\right)^{2}\right]|0\rangle_{e x} \times \\
\quad \exp \left\{-\frac{i}{\hbar} \sum_{n}\left[q_{n \alpha}(t) P_{n \alpha}-\pi_{n \alpha}(t) u_{n \alpha}\right]\right\}|0\rangle_{p h}
\end{gathered}
$$

where $\mathrm{L}$ is the coefficient of the chain-chain interactions among the three channels. From the time-dependent Schrödinger equation and the Heisenberg equations we can get

$$
\begin{aligned}
i \hbar \dot{a}_{n \alpha}(t)= & \varepsilon_{0} a_{n \alpha}(t)-J\left[a_{n+1 \alpha}(t)+a_{n-1 \alpha}(t)\right]+\chi_{1}\left[q_{n+1 \alpha}(t)-q_{n-1 \alpha}(t)\right] a_{n \alpha}(t)+\chi_{2}\left[q_{n+1 \alpha}(t)-q_{n-1 \alpha}(t)\right] \times \\
& {\left[a_{n+1 \alpha}(t)+a_{n-1 \alpha}(t)\right]+\frac{5}{2}\left\{W(t)-\frac{1}{2} \sum_{m}\left[q_{m \alpha}(t) \pi_{m \alpha}(t)-\dot{\pi}_{m \alpha}(t) \dot{q}_{m \alpha}(t)\right]\right\} a_{n \alpha}(t)+L\left[a_{n \alpha+1}(t)+a_{n \alpha-1}(t)\right] } \\
M \ddot{q}_{n \alpha}= & w\left[q_{n+1 \alpha}(t)-2 q_{n \alpha}(t)+q_{n-1 \alpha}(t)\right]+2 \chi_{1}\left[\left|a_{n+1 \alpha}(t)\right|^{2}-\left|a_{n-1 \alpha}(t)\right|^{2}\right]+ \\
& 2 \chi_{2}\left\{a_{n \alpha}^{*}(t)\left[a_{n+1 \alpha}(t)-a_{n-1 \alpha}(t)\right]+a_{n \alpha}(t)\left[a_{n+1 \alpha}^{*}(t)-a_{n-1 \alpha}^{*}(t)\right]\right\}
\end{aligned}
$$

Applying the method and way and fourth-order Runge-Kutta method [147-148] mentioned above we can simulate numerically the features of the solutions of Eqs. (28) - (31). In this case the initial condition of $a_{n \alpha}(t)=A \sec h\left[\left(n \alpha-n_{0} \alpha\right)\left(\chi_{1}+\chi_{2}\right)^{2} / 4 J w\right]$ was used in the calculation, where $\alpha=1,2,3, \mathrm{~A}$ is the normalization factor. Meanwhile we used $\bar{M}=5.73 \times 10^{-25} \mathrm{~kg}=114 \times 3 \mathrm{amu}$ (atomic mass units), $\mathrm{W}=39 \mathrm{~N} / \mathrm{m}, \varepsilon_{0}=0.2035 \mathrm{eV}$, $\bar{J}=9.68 \times 10^{-4} \mathrm{eV}, \bar{\chi}_{1}=6.2 \times 10^{-11} \mathrm{~N}, \bar{\chi}_{2}=(10-18) \times 10^{-12} \mathrm{~N}$ and $\bar{L}=1.5 \mathrm{meV}$ for the $\alpha$-helix protein molecules in the simulation. 
The numerical solutions of Eqs. (28)-(29) for the $\alpha$-helix protein molecules with three channels are shown in figure 5 . In figure 7 a we show the motion behaviors of the solution, where the initial condition of $a_{n \alpha}(t)=A \sec h\left[\left(n \alpha-n_{0} \alpha\right)\left(\chi_{1}+\chi_{2}\right)^{2} / 4 J w\right]$ is simultaneously motivated on the first ends of the three channels. From this figure we see that this solution can retain a clock shape while moving over a long distance in the range of the spacing of 400 amino acid residues at a time of $40 \mathrm{ps}$ without dispersion along the molecular chains, i.e., this solution is a soliton. This is similar to the above analytic and numerical results for the proteins with a single chain. In figure $7 \mathrm{~b}$ and $7 \mathrm{c}$ we plot the features of the solutions, with the initial conditions: $\mathrm{a}_{\mathrm{na}}(\mathrm{t}=0)=0$, here $\alpha=1,2, a_{n 3}(t)=A \sec h\left[\left(n-n_{0}\right)\left(\chi_{1}+\chi_{2}\right)^{2} / 4 J w\right]$ and $a_{n \alpha}(t)=A \sec h\left[\left(n-n_{0}\right)\left(\chi_{1}+\chi_{2}\right)^{2} / 4 J w\right]$ ' where $\alpha=1,2, \quad a_{n 3}(t=0)=0$ are used, respectively. These initial conditions denote that the first ends of one channel and two channels are motivated, and that the other two channels and the single channel are not linked, respectively. Figure $7 \mathrm{~b}$ indicates that two new waves with small amplitudes are generated due to the mutual interactions among the three channels, except for one soliton occurring on the channel linked by initial conditions. Although the two excitations are small, they can move over long distances along the two chains while keeping their amplitudes. Therefore, they are still solitons with small amplitudes. However, there exists a strange phenomenon in figure $7 \mathrm{c}$, in which the amplitudes of solitons generated in the two motivated chains are small, but the soliton in an unmotivated chain is larger, obviously, it is due to the superposition of waves induced by the other two chains. From this study we know that the solitons formed have higher energy when the initial conditions are simultaneously motivated on the first ends of the three channels.

We further study the collision property of solitons, set up from opposite ends of the channels for $\alpha$-helix protein molecules; the result is shown in figure 8a, where the above initial conditions simultaneously motivate the opposite ends of the three channels, where initial two solitons separating 100 amino acid spacings in each channel collide with each other at about $17 \mathrm{ps}$. After this collision, the two solitons in each channel go through each other without scattering to propagate toward and separately along the three chains, satisfying the rules on collision of macroscopic particles, which demonstrates that the solitons have a corpuscle feature. In figure

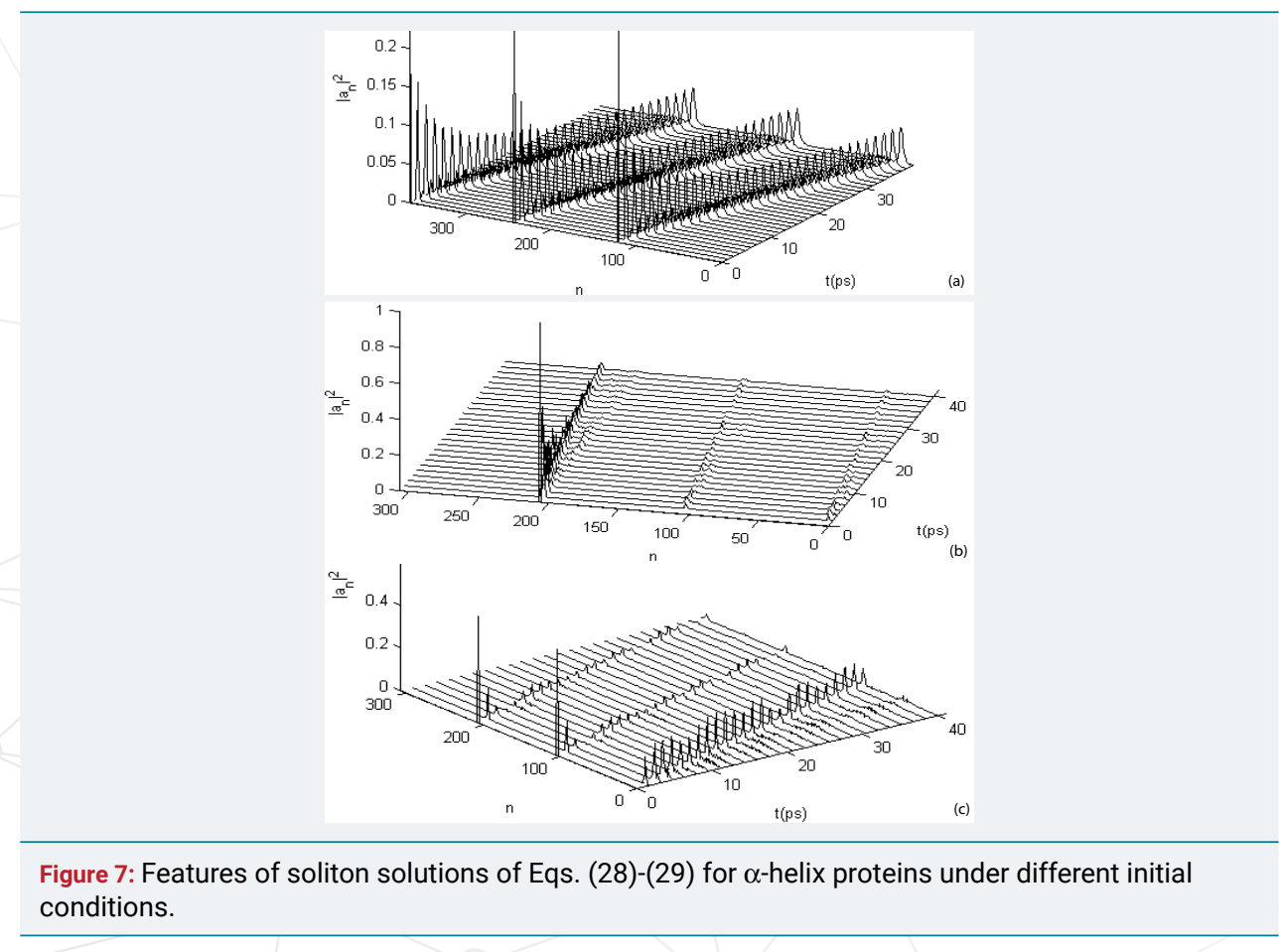


$8 \mathrm{~b}$ and $8 \mathrm{c}$ we plot the collision features of the two solitons generated from opposite ends of the channels with the above initial conditions motivating the opposite ends of one channel and two channels, and where they are not linked with the opposite ends of the other two channels and the single channel, respectively. In the two cases the soliton feature of the solutions after collision is substandard, especially where the initial condition motivates only the opposite ends of the single channel, as shown in figure 8b. Thus, the solutions of Eqs.(28)-(29) have a better soliton feature, where the opposite ends of the three channels are motivated simultaneously by the above initial conditions in the $\alpha$-helix protein molecules. Hence, the soliton excited in the $\alpha$-helix protein molecules has higher stability in the case of simultaneous motivation of the initial condition on the three channels.

In figure 9 we show the states of soliton solutions of Eqs. (30) - (31) at a time of $120 \mathrm{ps}$ and at 300 amino acid spacings, where the above initial conditions are simultaneously linked on the first ends of the three channels. We see that the soliton still retains its amplitude and shape while moving. This result shows that the lifetime of the soliton is at least 120Ps., thus it is very stable.

The above results show that the states of Pang's soliton are stable in an uniform protein molecular chain, thus the bio-energy transport can be carried out in this system.

On the other hand, Förner [149] studied also the states of Davydov soliton in $\alpha$-helix proteins with three channels using wave function, in which he used the dynamic equations in Eqs.(12)-(13) at $2 \chi_{2}=0$ and $\frac{5}{2}\left\{W(t)-\frac{1}{2} \sum_{m}\left[q_{m \alpha}(t) \pi_{m \alpha}(t)-\dot{\pi}_{m \alpha}(t) \dot{q}_{m \alpha}(t)\right]\right\} a_{n \alpha}(t)$ $=0$, but $\chi_{1}$ is replaced by $\chi$ in Eqs.(12)-(13), where the symmetric A model $\left(a_{n 1}=a_{n 2}=a_{n 3}\right)$, the linear combination of the two degenerate $E$ modes $\left(a_{n 1}=0, a_{n 2}=a_{n 3}=-1 / \sqrt{2}\right)$ and an asymmetric local excitation(L mode) of one unit on a single chain are used. In this case the result of numerical simulation for Davydov soliton is shown in figure 10, where $\mathrm{L}=1.54 \mathrm{meV}, \mathrm{w}=13 \mathrm{~N} / \mathrm{m} . \mathrm{M}=114 \mathrm{~m}_{\mathrm{p}}, \mathrm{J}=0.967 \mathrm{meV}$ and $\chi=62 \mathrm{PN}$ were utilized. Figure 10 indicates that in the case of the A mode the soliton consists of three identical parallelmoving localized excitations on all three chains, while, in the E mode the soliton moves only on two chains. In the case of the local excitation the soliton is found mainly on one chain, with a small fraction of the excitation transferred to the others, but the

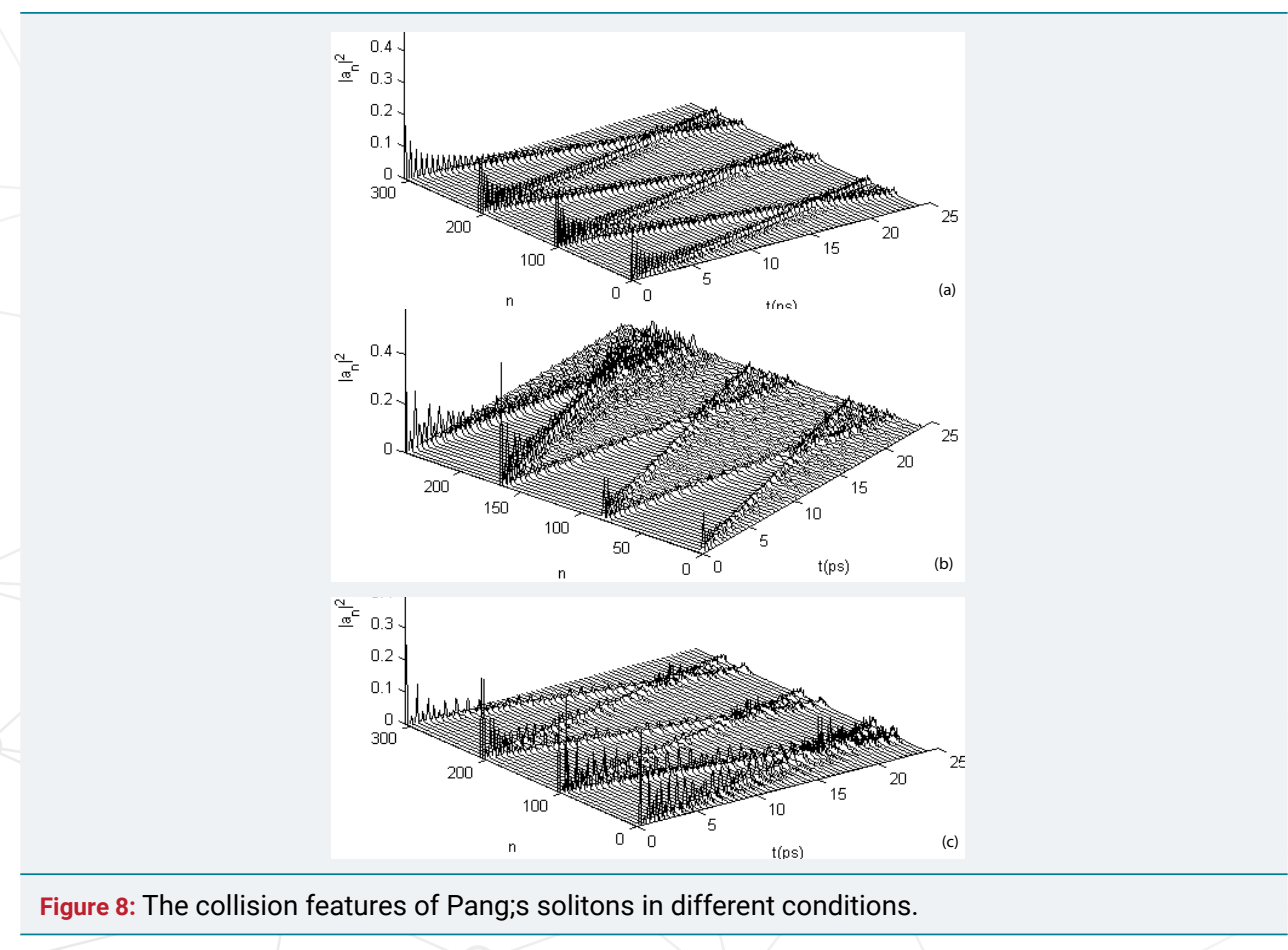




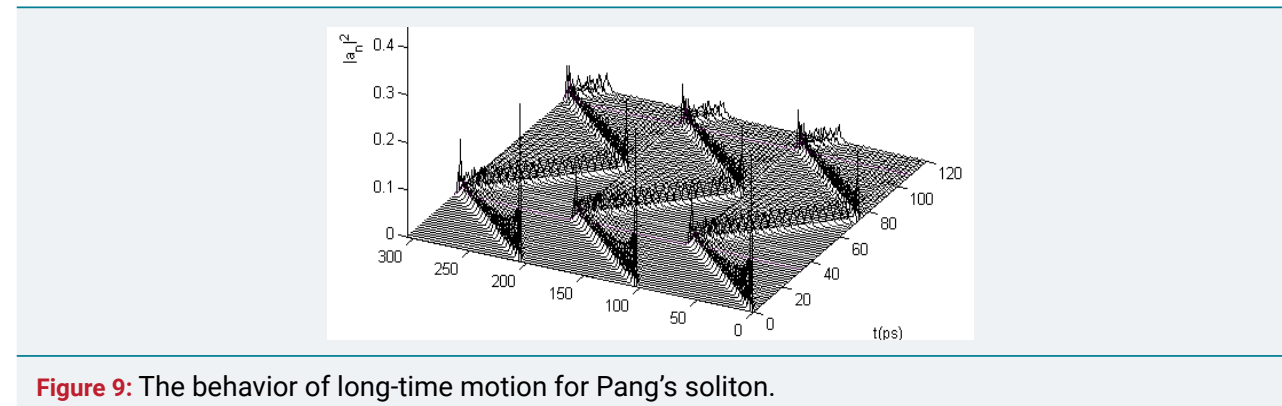

Figure 9: The behavior of long-time motion for Pang's soliton

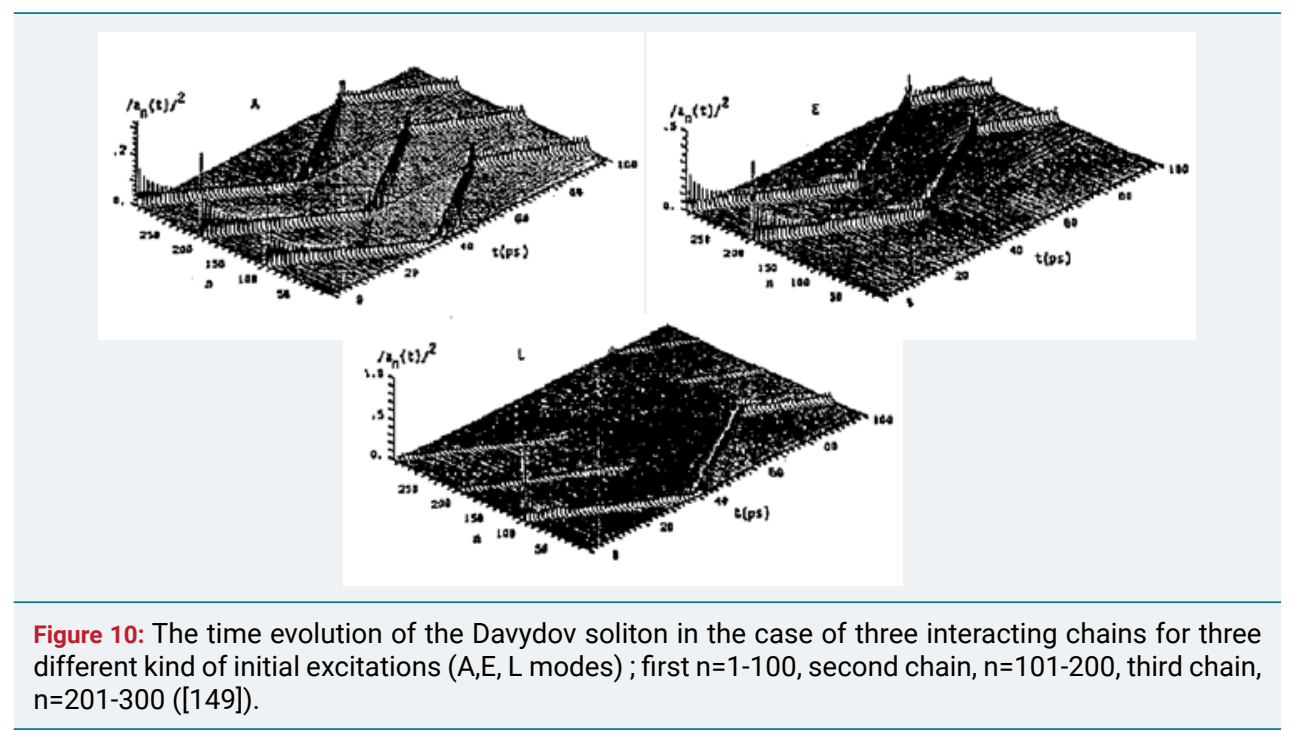

phenomenon reported by Scott $[15,18]$ that solitons on one chain jump to another after some time have not observed. Maybe the built-up of a small fraction of the excitation on the other chains in the case of the asymmetric initial condition might lead to a transfer of the whole soliton after longer times.

Influence of structure nonuniformity on the energy transport in protein molecules

\section{The results in Davydov model}

As mentioned above, Förner [50] obtained that Davydov soliton with $\left|D_{2}\right\rangle$ is stable at $\chi_{1}=100 \mathrm{PN}$, which is larger than the widely accepted value of $62 \mathrm{PN}$. Thus he study further the influences of structure nonuniformity on the states of the Davydov soliton with $\left|D_{2}\right\rangle$ in a single chain protein molecule using Eqs.(23)-(24) and the fourth-order Runge-Kutta method [147-148] in this case.

As mentioned above, protein molecules consist of 20 different amino acid molecules with weights between $75 \mathrm{~m}_{\mathrm{p}}$ (glycine) and $204 \mathrm{~m}_{\mathrm{p}}$ (tryptophane), which correspond to weight variations between $0.67 \overline{\mathrm{M}}$ and $1.80 \overline{\mathrm{M}}$. Therefore it is an aperiodic system, and there is a disordered distribution in mass sequences of amino acids. To study structure nonuniformity arising from the mass disorder distribution of amino acid residues, which results necessarily in changes of characteristic parameters in the dynamic equations in Davydov's model, Förner introduced a random number generator to create random sequences of the mass along the chain. Disorder in the mass sequence destroys the soliton only for a very large disorder strength, with $M_{n}$ values $0.01 \bar{M}<M_{n}<50 \bar{M}$. For $0.01 \bar{M}<M_{n}<10 \bar{M}$ the soliton velocity is reduced from 0.73 to $0.59 \mathrm{~km} / \mathrm{s}$, the sound velocity to $2.12 \mathrm{~km} / \mathrm{s}$. In the case of the mass variation of natural amino acids $\left(0.67 \bar{M}<M_{n}<1.80 \bar{M}\right)$ no change in soliton dynamics is virtually found, thus the average mass approximation is justified. For the change of $\mathrm{w}_{\mathrm{n}^{\prime}}$, he found 
no change in soliton dynamics up to random variation of $\pm 20 \% \overline{\mathrm{w}}$. For $\pm 30 \% \overline{\mathrm{W}}$ the soliton velocity is somewhat reduced to $0.68 \mathrm{~km} / \mathrm{s}$. Finally the soliton disperses slowly and its propagation is irregular for $\pm 40 \% \overline{\mathrm{W}}$.

For the fluctuations of $\mathrm{J}$ alone or together with the natural mass variation the soliton is stable up to $\pm 5 \% \bar{J}$. Therefore, Davydov soliton is far more sensitive to variation in $\mathrm{J}_{\mathrm{n}}$ than in other parameters. If in addition $\mathrm{W}_{\mathrm{n}}$ is aperiodic, the soliton is stable up to $\pm 10 \% \overline{\mathrm{W}}$, while at $\pm 20 \% \overline{\mathrm{W}}$ slowly dispersive behavior appears. Finally, if $\chi_{1 n}$, alone is aperiodic, or if $\chi_{1 n}$ is aperiodic together with the natural mass variation, $\chi_{1 n}$, can be varied up to $\pm 20 \% \bar{\chi}_{1}$ without destruction of the soliton. However, if disorder in $w_{n}$ is also introduced, $\chi_{1 n}$ can be varied up to $\pm 15 \% \bar{\chi}_{1}$ and $\mathrm{w}_{\mathrm{n}}$ up to $\pm 40 \% \mathrm{~W}$. Finally if all four parameters are randomly varied the maximal possible disorder that would still allow the existence of a soliton is $\pm 20 \% \overline{\mathrm{w}}, \pm 2.5 \% \mathrm{~J}$, and $\pm 10 \% \bar{\chi}_{1}$. For this disorder strength he has calculated 10 different randomly chosen sequences to find out whether the soliton properties depend only on the magnitude of disorder or also on the individual sequences. He found that only the soliton velocity is affected; in this case it varies between 0.61 and $0.80 \mathrm{~km} / \mathrm{s}$.

Figure 11 shows the effects of disorder in the sequence of masses of amino acid residues on the Davydov soliton by Förner[59], in which the mass at site 100 is increased to $100 \overline{\mathrm{M}}$ and $200 \overline{\mathrm{M}}$, respectively, but all other masses have been still kept equal to $\overline{\mathrm{M}}$. We find that no visible perturbation occurs in the soliton motion, a small fraction of the sound energy is trapped at the impurity and the major fraction is scattering back This denoted that an impurity at one site, which may be some other molecules bound to the protein at this site (like reactive centres as e.g. heme groups) does not disturb obviously the soliton, unless it does not influence the coupling constant. In figure 12 he gave the influences of a random series of masses for the whole chain, $\alpha_{k} \overline{\mathrm{M}}$, on the Davydov soliton, where $\alpha$ is a random-number generator with equal probability within a prescribed interval and is in the ranges of $0.67 \leq \alpha_{k} \leq 1.80$ and $0.67 \leq \alpha_{k} \leq 200$. Figure 10 shows that the aperiodicity due to the first smaller intervals for $\alpha_{k}$ does not significantly affect the Davydov soliton motion, but in the case of the larger intervals, the soliton disperses.
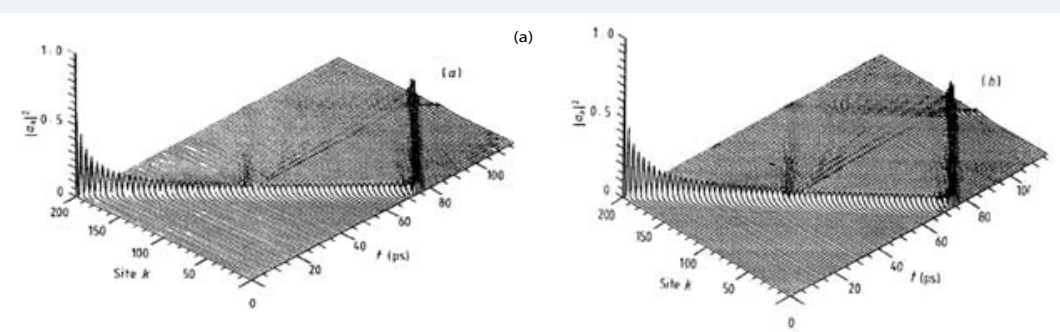

Figure 11: The evolution of Davydov soliton as function of site and time in a single chain protein with an impurity at site 100 for different impurity massesof $100\left|D_{2}\right\rangle(\mathrm{a})$ and $200 \overline{\mathrm{M}}$ (b), respectively.
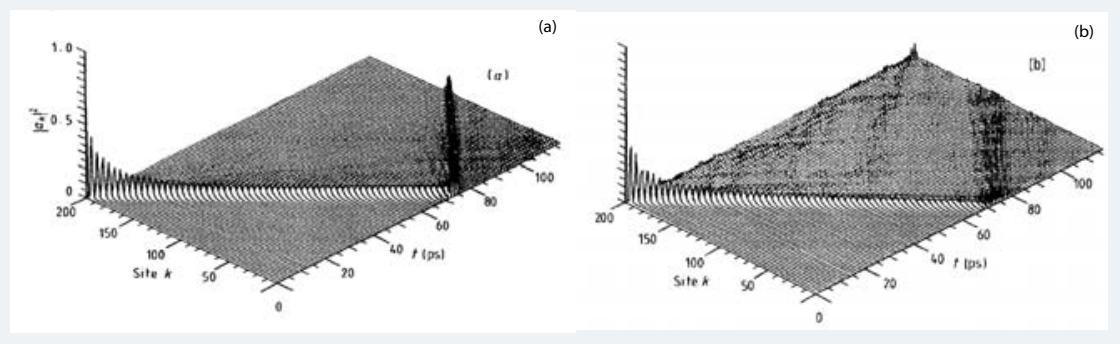

Figure 12: The evolution of Davydov soliton as function of site and time in a single chain protein with an random mass sequence $\left|D_{2}\right\rangle$ for different intervals of the $\overline{\mathrm{M}}, 0.67 \leq \alpha_{k} \leq 1.80$ (a) and $0.67 \leq \alpha_{k} \leq 200$ (b). 
Figure 13 shows Förner's six results for the moved states of Davydov soliton under different fluctuations of $\mathrm{W}, \mathrm{J}$ and $\chi_{1 n}$ (or $\mathrm{x}_{\mathrm{k}}$ ) in the case of $0.67 \bar{M} \leq M \leq 1.80 \bar{M}$ and $\bar{\chi}_{1}=62 P N \quad[50,150]$. Up to a random variation of $\pm 20 \% \overline{\mathrm{W}}$, we see no changes in the dynamics of the soliton. For $\pm 30 \% \overline{\mathrm{W}}$ (Figure 13a) the soliton velocity is somewhat reduced to $0.68 \mathrm{~km} / \mathrm{s}$. Finally, for $\pm 50 \% \mathrm{~W}$ (Figure 13b) the excitation decreases slowly and the propagation is irregular. Figure 13c shows the state of the soliton in the case of the fluctuations of $\pm 5 \% \bar{J}$. and $\pm 10 \% \mathrm{~W}$ in which the soliton is stable, but it disperses slowly at $\pm 20 \% \mathrm{~W}$ as shown in figure $13 \mathrm{~d}$. If disorder also in $\mathrm{w}_{\mathrm{n}}$ is introduced, the soliton is stable, when $\chi_{1 n}$ can be varied up to $\pm 15 \% \bar{\chi}_{1}$ and $\mathrm{w}_{\mathrm{n}}$ up to $\pm 40 \% \overline{\mathrm{W}}$ (Figure 13e). If finally, all parameters are randomly varied the maximal possible disorder which still allows the existence of a soliton is $\pm 20 \% \overline{\mathrm{W}} \pm 2.5 \%, \bar{J}$ and $\pm 10 \bar{\chi}_{1}$. Figure $11 \mathrm{f}$ shows a dispersive example of the soliton at $\pm 15 \% \overline{\mathrm{W}}, \pm 5 \%, \bar{J}$ and $\pm 15 \% \bar{\chi}_{1}$.

The case of diagonal disorder $\varepsilon_{n}$ he found that for an isolated impurity in the middle of the chain $\left(\varepsilon_{n}=\varepsilon \delta_{n, 100}\right)$ the soliton can pass the impurity only if $\varepsilon<0.5 \mathrm{meV}[50,150]$. In other cases it is rejected or destroyed. In the case of a random sequence ( $\varepsilon_{n}=\varepsilon \beta_{n},\left|\beta_{n}\right|<1, \beta n$ is random) only for $\varepsilon<1 m e$ can the soliton pass the chain. For higher values of $\varepsilon$ the excitation disperses quickly.

Therefore, we can obtained from Förner's study that the Davydov soliton in wave function $\left|D_{2}\right\rangle$ remains stable against strong disorder in the sequences of masses, spring constants, and coupling constants. But weak diagonal disorder and small dipole-dipole interaction constants can all destroy the solitons.

In the meanwhile, Förner [50,150-152] studied also the influences of structure nonuniformity on the states of the Davydov soliton with wave function $\left|D_{1}\right\rangle$, in which the quantum nature of the lattice plays a greater role than in the classical $\left|D_{2}\right\rangle$ state, Thus he obtained that the Davydov soliton appears only from nonlinearities roughly 3
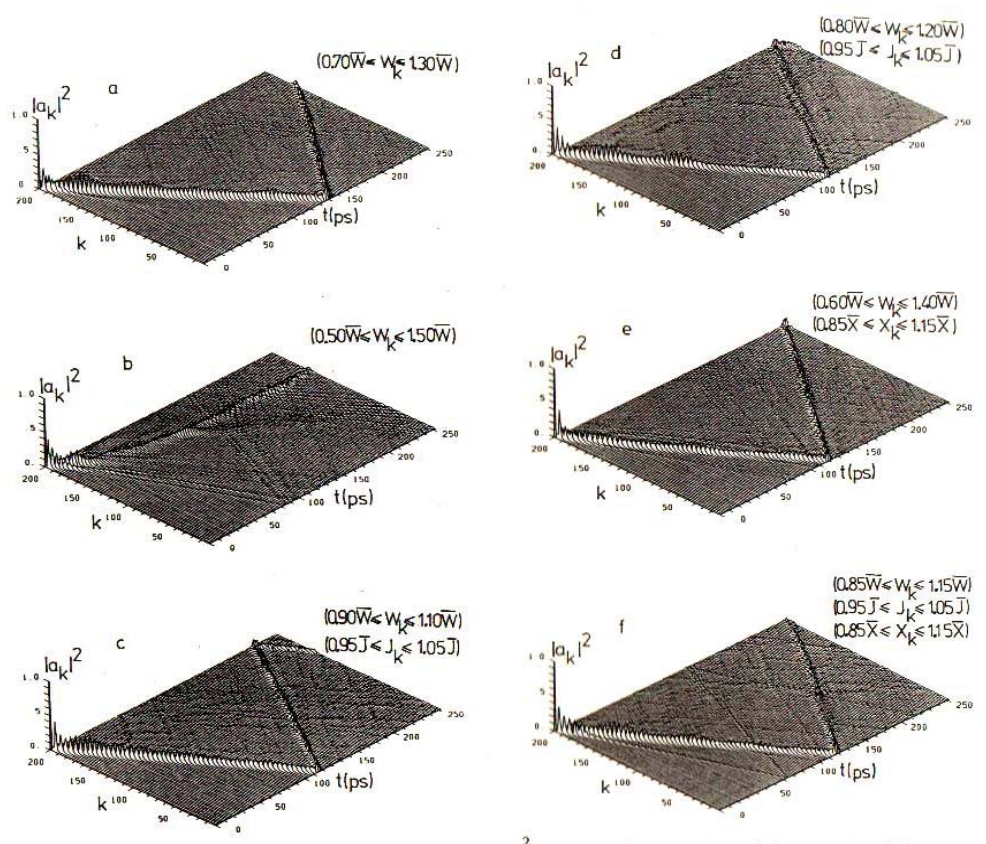

Figure 13: The states of motion of Davydov soliton as function of site $(k)$ and time $(\mathrm{t})$ for some typical examples of nonuniformity at $0.67 \bar{M} \leq M \leq 1.80 \bar{M}$ and $\overline{\chi_{1}}=62 P N$ (see Förner's work [150]. 
to 4 times larger than those in $\left|D_{2}\right\rangle$ models, for example the Davydov soliton with $\left|D_{1}\right\rangle$ ansatz is stable at $\chi_{1}=140 \mathrm{PN}$, which is more larger than the widely accepted value of $62 \mathrm{PN}$. The sensitivity of such solitons to these disorders is practically opposite that for the $\left|D_{2}\right\rangle$ state. Thus it is not necessary to study further the influences of structure nonuniformity of protein molecules on the states and properties on Davydov's solitons.

\section{The results in Pang's model}

\section{The results in single chain protein}

In order to study the influence of a random series of masses on Pang's soliton, Pang introduced a parameter $\alpha_{\mathrm{k}^{\prime}}$ representing the size of the disorder distribution, which is a random-number generator with equal probability within a prescribed interval and can denote the mass at each point in the molecular chain, i.e. $\mathrm{M}_{\mathrm{k}}=\alpha_{k} \overline{\mathrm{M}}$ [38-46]. Numerical simulation shows that when the $\alpha_{\mathrm{k}}$ is small, such as, an interval $0.67 \overline{\mathrm{M}} \leq$ $M_{k} \leq 1.80 \overline{\mathrm{M}}$, the state of Pang's soliton is not influenced. Up to the $\alpha_{\mathrm{k}}$ intervals of, for example, $0.67 \leq \alpha_{\mathrm{k}} \leq 300$, the stability of Pang's soliton may still remain, but in the case of large intervals such as $0.67 \leq \alpha_{k} \leq 700$, the vibrational energy is dispersed, as shown in figure 14. The interval of $0.67 \leq \alpha_{k} \leq 300$, over which the motion of Pang's soliton is unperturbed, is evidently larger than the above natural interval of masses of amino acids. Thus, Pang's soliton is very robust against mass disorder in proteins.

However, in the above calculation we do not introduced the fluctuations of the spring constant $w$, dipole-dipole interaction constant J, coupling constant $\left(\chi_{1}+\chi_{2}\right)$, and the ground state energy $\varepsilon_{0}$ arising from the structure nonuniformity. According to the above Förner's method [50,150-152] the changes in parameters are represented by the fluctuations of the average increments, $\Delta \mathrm{w}=\mathrm{w}-\overline{\mathrm{W}}, \Delta \mathrm{J}=\mathrm{J}-\overline{\mathbf{J}}, \Delta\left(\chi_{1}+\chi_{2}\right)=\left(\chi_{1}+\chi_{2}\right)-($ $\left.\bar{\chi}_{1}+\bar{\chi}_{2}\right)$ and $\Delta \varepsilon_{0}=\varepsilon_{0}-\varepsilon_{0^{\prime}}$ respectively, where $\mathrm{w}, \mathrm{J},\left(\left(\chi_{1}+\chi_{2}\right)\right.$ and $\varepsilon_{0}$ are the values of the parameters in the protein molecules with the structural disorders. However, for the variation in ground state energy arising from imported impurities or from the mass disorder, we use generally the random number generator, $\left|\beta_{n}\right|$, to designate its random feature, i.e., the $\Delta \varepsilon 0$ is denoted by $\Delta \varepsilon_{0}=\varepsilon\left|\beta_{n}\right|$. In the following we study the collective effects of the fluctuations of the parameters on Pang's soliton.

In figure 15. we show the changes in stability of Pang's soliton with increasing fluctuations of the spring constant $w$ in the case of a mass interval of $0.67 \leq \alpha_{k} \leq 2$. We see that up to a random variation of $\pm 40 \% \overline{\mathrm{W}}$, the dynamics of the soliton have not changed. For $\pm 50 \% \overline{\mathrm{W}}$, the soliton disperses somewhat, but its velocity is only somewhat diminished, when compared to the earlier $\overline{\mathrm{W}}$ case. Finally, for $\pm 70 \% \mathrm{~W}$, the soliton disperses and its propagation is irregular,as shown in figure 15.

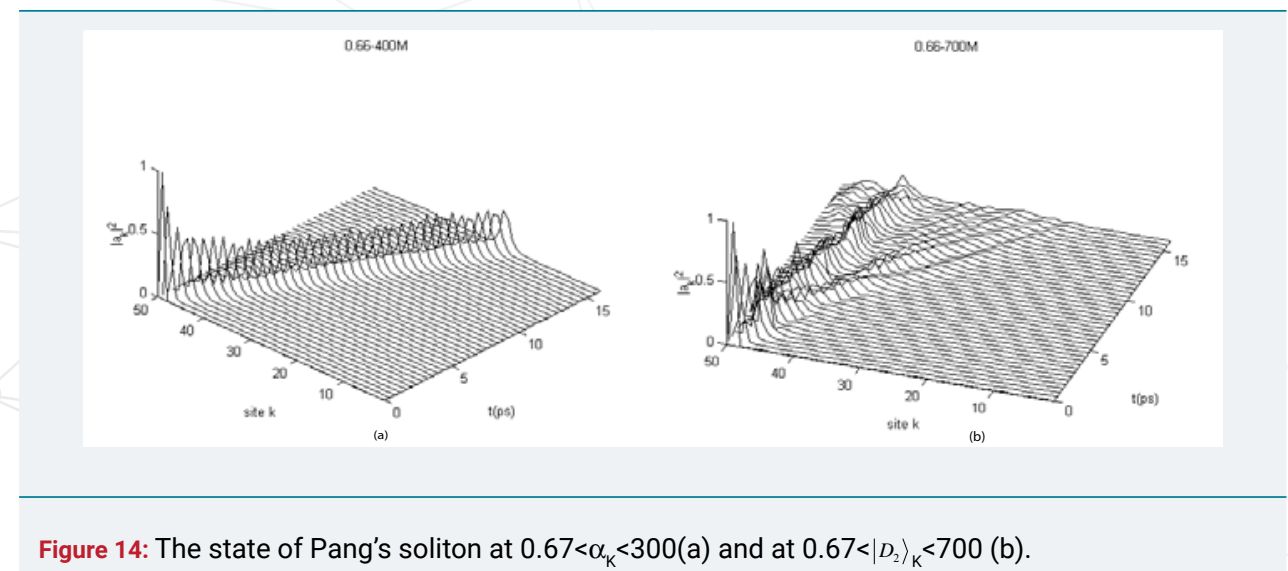




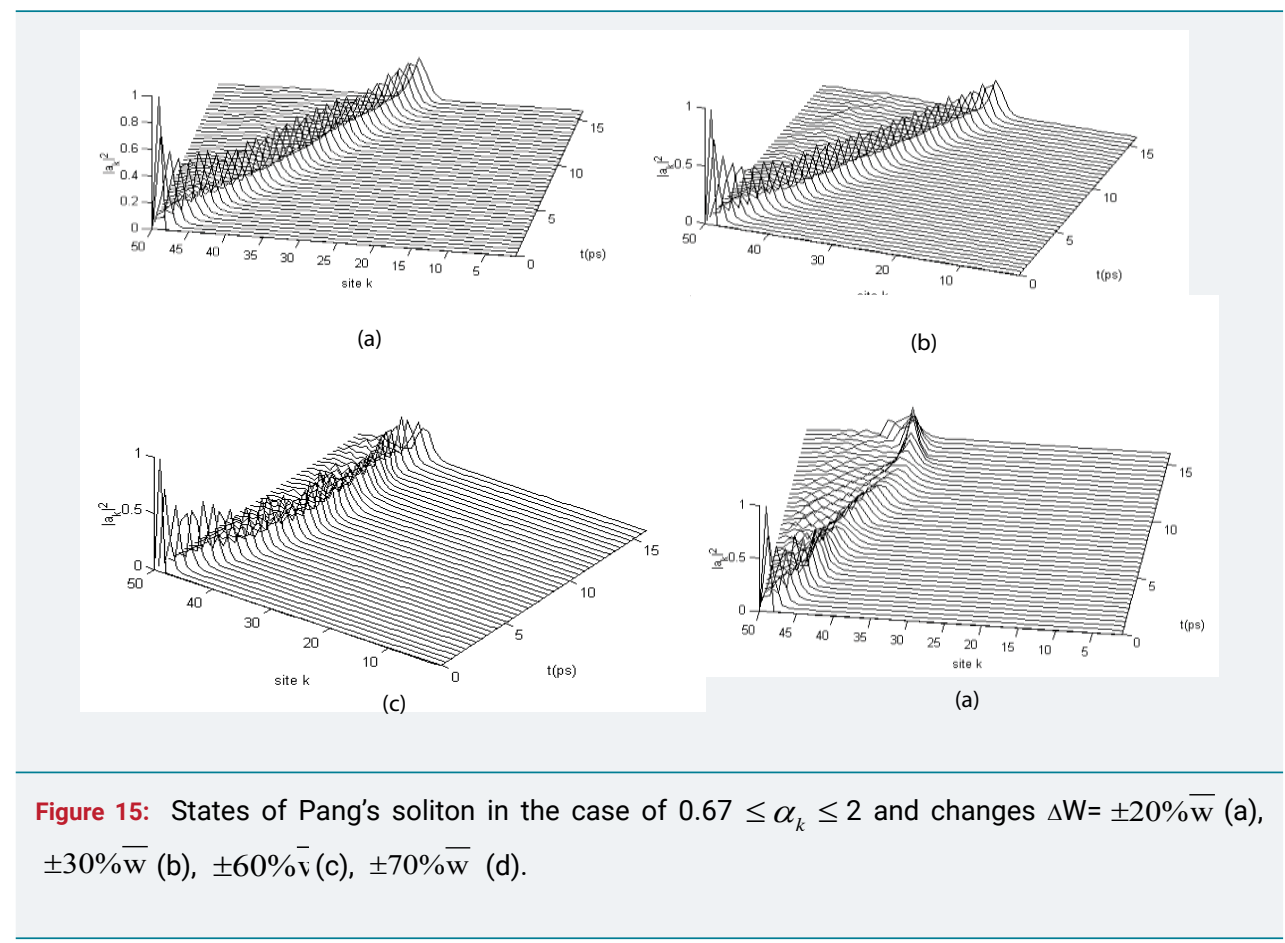

The soliton in Pang's model [113-125] is also more sensitive to the variations in the dipole-dipole interaction constant J caused by the structural disorder when compared with the other parameters. The simulation shows that for a variation in J alone, the soliton is stable up to $\pm 9 \% \bar{J}$, and it disperses at $\Delta \mathrm{J}= \pm 15 \% \bar{J}$. If we simultaneously consider the collective effects of disorder of mass sequence and fluctuation of $\mathrm{J}$ on Pang's soliton, then its state is obviously changed. In figure 16 we show the collective effect of the fluctuations $\Delta \mathrm{J}= \pm 10 \% \bar{J},= \pm 20 \% \bar{J}$, on the soliton in the case of a mass interval of 0.672 . From these figures we see clearly that Pang's soliton is stable at $\Delta \mathrm{J} \leq 10 \% \bar{J}$, but it disperses significantly at $\Delta \mathrm{J}= \pm 20 \% \bar{J}$.

The numerical calculation shows that, arising from the disorder of structure, if the coupling constant $\left(\chi_{1}+\chi_{2}\right)$ alone is changed, $\left(\chi_{1}+\chi_{2}\right)$ can be varied to $\pm 25 \%\left(\bar{\chi}_{1}+\bar{\chi}_{2}\right)$, and in this case Pang's soliton does not disperse. However, for a fluctuation together with natural mass variation, the stability of Pang's soliton will be changed. In figure 17 we illustrate the changes in the states of new solitons with increasing fluctuations of $\left(\chi_{1}\right.$ and $\left.\chi_{2}\right)$ at $0.67 \bar{M} \leq \mathrm{M} \leq 2 \bar{M}$. We see from this figure that only at $\Delta\left(\chi_{1}+\chi_{2}\right)<$ $30 \%\left(\chi_{1}+\chi_{2}\right)$ Pang's solitons are stable, but they obviously disperse at $\Delta\left(\chi_{1}+\chi_{2}\right)=35 \%$ ( $\left.\bar{\chi}_{1}+\bar{\chi}_{2}\right)$

It is quite necessary to collect the combined effect of random variations of the above physical parameters resulting from structural disorders on the properties of Pang's soliton. The changes of states of solitons with increasing interaction constants in the case of $0.67 \leq \alpha_{\mathrm{k}} \leq 2$ and $\Delta \mathrm{J}= \pm 10 \% \bar{J}$ is shown in figure 18 . We find no change in the dynamics of Pang's soliton at $\Delta w= \pm 25 \% \overline{\mathrm{W}}$ and $\Delta \mathrm{J}= \pm 10 \% \bar{J}$, but it begins dispersing at $\Delta w= \pm 30 \% \overline{\mathrm{W}}, \Delta J= \pm 15 \% \bar{J}$, and disperses considerably at $\Delta w=$ $\pm 25 \% \overline{\mathrm{W}}, \Delta J= \pm 15 \% \bar{J}$.

We are more interested in the collective effect of simultaneous random-variations of the above five parameters resulting from structural nonuniformity on Pang's soliton. In general, Pang's soliton is very sensitive to the diagonal disorder, which is the change in ground state energy, $\Delta \varepsilon_{0}$ caused by different side groups of amino acids and local geometric distortions due to the impurities imported. We found that for an isolated impurity in the middle of the chain, which causes the change in the ground energy to be $\Delta \varepsilon_{0}=\delta_{n}$, the soliton can pass the impurity only if $\varepsilon<1 \mathrm{meV}$. In other cases it 


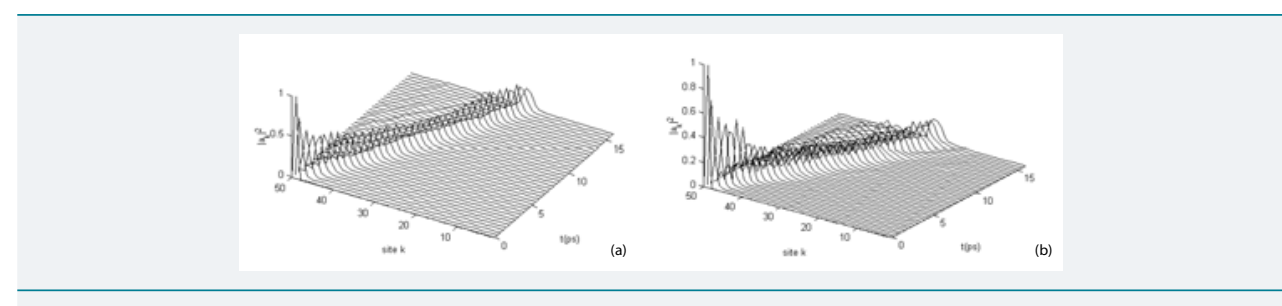

Figure 16: The states of Pang's soliton in the case of the mass interval $0.67 \leq \alpha_{k} \leq 2$ as it changes at $\Delta J= \pm 10 \% \bar{J}(\mathrm{a})$ and $\Delta J= \pm 20 \% \bar{J}(\mathrm{~b})$.
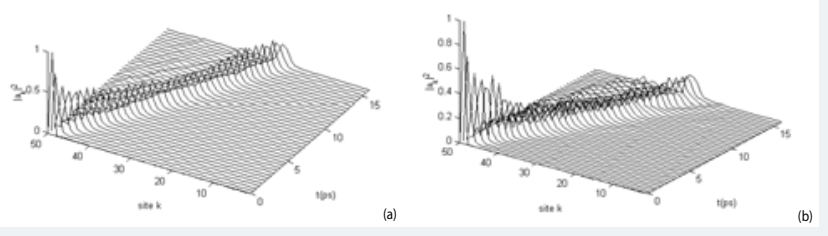

Figure 17: The states of Pang's soliton in the case of the mass interval 0.67 $\pm 30 \%\left(\overline{\chi_{1}}+\overline{\chi_{2}}\right)$ (a) and $\pm 35 \%\left(\overline{\chi_{1}}+\overline{\chi_{2}}\right)$ (b).

$\leq \alpha_{k} \leq 2$ as it changes at

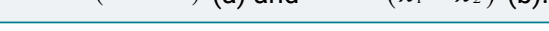
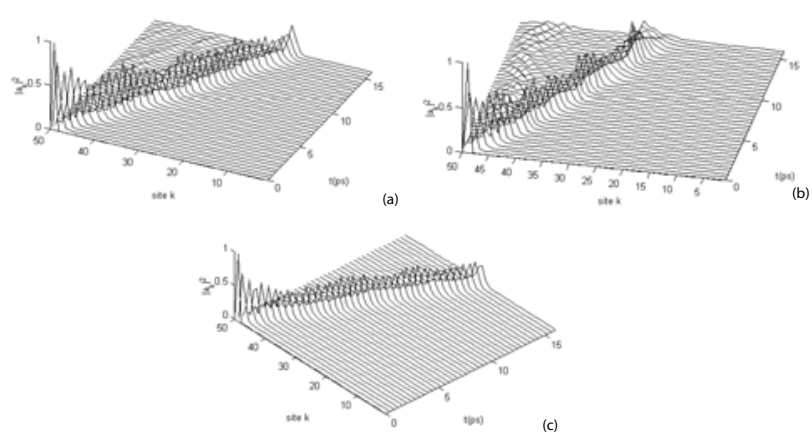

Figure 18: The stability of Pang's soliton at $0.67 \leq \alpha_{k} \leq 2$ and $\Delta\left(\chi_{1}+\chi_{2}\right)= \pm 10 \%\left(\overline{\chi_{1}}+\overline{\chi_{2}}\right)$ and $\Delta w= \pm 30 \% \overline{\mathrm{w}}, \Delta J= \pm 15 \% \bar{J}$ (a) and $\Delta w= \pm 25 \% \overline{\mathrm{w}}, \Delta J= \pm 15 \% \bar{J}$ (b) and $\Delta w=$ $\pm 25 \% \overline{\mathrm{w}}, \Delta J= \pm 10 \% \bar{J}$ (c).

is reflected or dispersed. In the case of a random sequence, $\Delta \varepsilon_{0}=\varepsilon\left|\beta_{n}\right|,\left|\beta_{n}\right| \leq 0.5, \beta_{n}$ is a random parameter, and only when $\varepsilon<1 \mathrm{meV}$ can the soliton pass the impurity to propagate along the chains. For higher values of $\varepsilon$ the soliton is dispersed. When the diagonal disorder occurs together with fluctuations of the other four parameters, the state of Pang's soliton is changed obviously. The states of solitons with an increasing spring constant in the cases of $0.67 \leq \alpha_{\mathrm{k}} \leq 2, \Delta\left(\chi_{1}+\chi_{2}\right)=5 \%\left(\bar{\chi}_{1}+\bar{\chi}_{2}\right), \Delta \mathrm{J}= \pm 5 \% \bar{J}$ and $\Delta \varepsilon_{0}=\varepsilon\left|\beta_{n}\right|,\left|\beta_{n}\right| \leq 0.5, \quad \varepsilon<1 \mathrm{meV}$ are given in figure 19. We see from this figure that when the five parameters are all randomly varied, a maximal disorder that would occur still with the soliton in motion, is $\Delta \mathrm{w}= \pm 10 \% \mathrm{~W}, \Delta \mathrm{J}= \pm 5 \% \bar{J}, \Delta\left(\chi_{1}+\chi_{2}\right)= \pm 5 \%\left(\bar{\chi}_{1}+\bar{\chi}_{2}\right.$ ), $0.67 \bar{M} \leq \mathrm{M} \leq 2 \overline{\mathrm{M}}$ and $\Delta \varepsilon_{0}=\varepsilon\left|\beta_{n}\right|,\left|\beta_{n}\right| \leq 0.5, \quad \varepsilon=1 \mathrm{meV}$. In other cases the soliton is dispersed or reflected by the impurity.

In summary, we have looked at the stability of Pang's solitons under the influence of various structural disorders in the proteins via the fourth-order Runge-Kutta method [23-24] which can help identify and check the stability of state of Pang's 

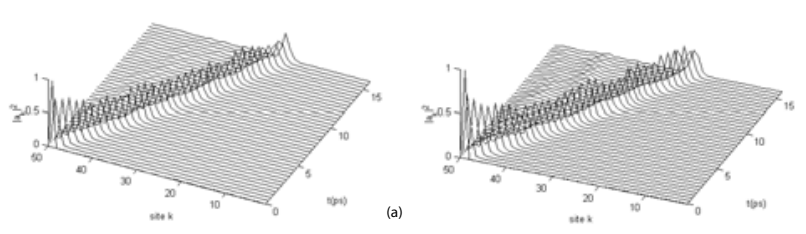

Figure 19: The states of Pang's soliton at $\Delta \varepsilon_{0}=\varepsilon\left|\beta_{n}\right|,\left|\beta_{n}\right| \leq 0.5, \varepsilon<1 \mathrm{meV}$ and $\Delta J= \pm 5 \% \bar{J}$, $\Delta\left(\chi_{1}+\chi_{2}\right)= \pm 5 \%\left(\overline{\chi_{1}}+\overline{\chi_{2}}\right), 0.67 \leq \alpha_{k} \leq 2$ for $\Delta w= \pm 10 \% \overline{\mathrm{w}}$ (a), $\Delta w= \pm 20 \% \overline{\mathrm{w}}$ (b).

soliton transporting bio-energy. The above results obtained show that the soliton in Pang's model [113-125] is very robust against these structural disorders. However, the Davydov soliton with one quantum is not so, and smaller structural disorders in the proteins will destroy its stability as mentioned above, which are obtained by Forner er al. [50,149-152], what are the reasons why the soliton in Pang's model is comparably more stable than Davydov's? Clearly, this is due to the fact that the Pang model with a quasi-coherent two-quanta state differs from the Davydov model with one quantum state. Although Eqs. (25)- (27) can become the dynamic equations in the Davydov model when $\chi_{2}=0$ and $\sqrt{2} a_{n}$ are replaced by $A_{n}$ (in such a case, the normalization condition of the Davydov wavefunction then becomes $\sum\left|A_{n}(t)\right|^{2}=2 \sum\left|a_{n}(t)\right|^{2}=2$. This again shows clearly that the new wavefunction in Pang model contains exactly two quanta), the stability of Pang's soliton is greatly increased due to considerable increases of nonlinear coupling energy, $G_{p}$, and a binding energy, $E_{B P}$, which are larger by about three and twenty times than Davydov's soliton[113-125].

The results in $\alpha$-helix protein molecules with three channels in Pang's model.

We investigated further the properties of Pang's soliton in $\alpha$-helix protein molecules with three channels by fourth order Runge-Kutta method [147-148] and .using the above methods.

(1) The individual influences of different fluctuations and disorders on the motion of Pang's soliton.

(2) In accordance with the above method we study the influences of the random series of masses, in which whether of the smaller or larger intervals for $\alpha_{\mathrm{k}^{\prime}}$ such as, $0.67 \leq \alpha_{k} \leq 100$ or $0.67 \leq \alpha_{k} \leq 300$, on Pang's soliton. This result manifests that this nonuniformity does not significantly affect the stability of Pang's soliton, its vibrational energy is not dispersed as shown in figure 20, where other parameters take mean values mentioned above. The interval over which Pang's soliton moves unperturbed is evidently larger than the variation in the masses of the natural amino acids $\left(0.67 \leq \alpha_{k} \leq 1.80\right)$. Therefore, the soliton in Pang's model is very robust against mass nonuniformities in $\alpha$-helix protein molecules [113-123].

Numerical simulation results show that we do not find any changes in the dynamics of the soliton up to a random fluctuation of $\pm 25 \% \overline{\mathrm{W}}$. The results of our calculations are shown in figure 21 , in the cases where fluctuations are $\pm 15 \% \overline{\mathrm{W}}$ and $\pm 25 \% \overline{\mathrm{W}}$ , respectively. For $\pm 30 \% \overline{\mathrm{W}}$, Pang's soliton velocity is only somewhat diminished when compared with the case of $\overline{\mathrm{W}}$. Finally, for $\pm 40 \% \overline{\mathrm{W}}$, Pang's soliton disperses completely and propagation is irregular.

The soliton is very sensitive to variations in the dipole-dipole interaction J in the $\alpha$-helix protein molecules. The soliton is stable up to $5 \%$ as shown in figure 22 , where we denote the features of the solitons under changes of $\pm 3 \% \bar{J}$ and $\Delta \mathrm{J}= \pm 5 \% \bar{J}$, but the soliton disperses at $\Delta \mathrm{J}= \pm 7 \% \bar{J}$. 


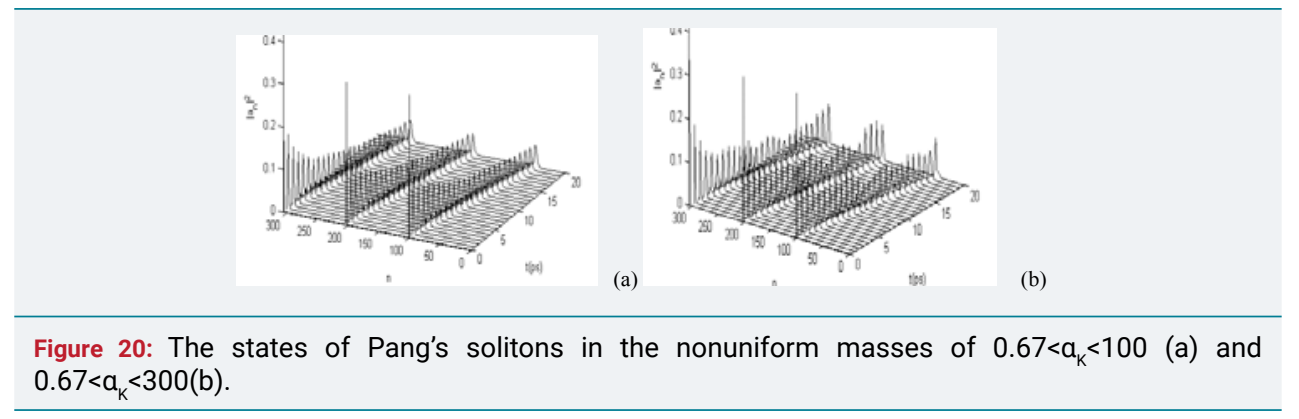
$0.67<a_{K}<300(b)$.
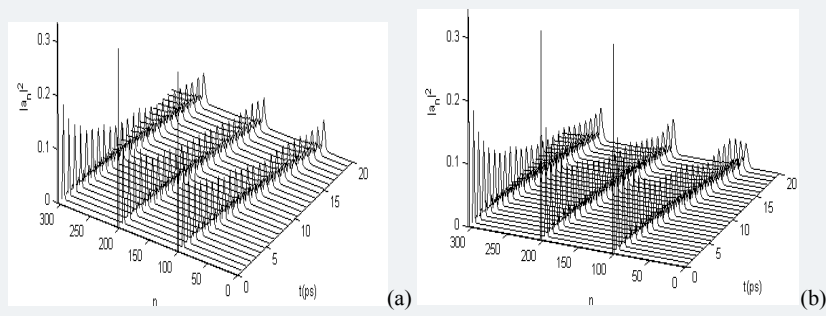

Figure 21: The states of Pang's solitons at the fluctuations of $\Delta W= \pm 15 \% \bar{W}(a)$ and $\Delta W= \pm 25 \% \bar{W}(b)$.
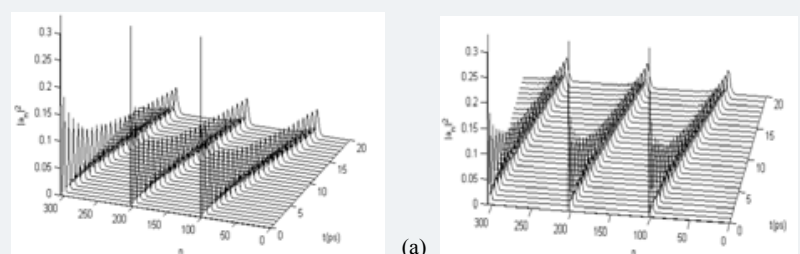

(a)

(b)

Figure 22: The features of Pang's solitons under the changes $\Delta \mathrm{J}= \pm 3 \% \bar{J}$ (a) and $\Delta \mathrm{J}= \pm 5 \% \bar{J}$ (b).

In figure 23 we exhibit the changes of feature of Pang's soliton under the influence of fluctuations of $\Delta\left(\chi_{1}+\chi_{2}\right)= \pm 6 \%\left(\bar{\chi}_{1}+\bar{\chi}_{2}\right)$ and $\pm 9 \%\left(\bar{\chi}_{1}+\bar{\chi}_{2}\right)$.These figures show that Pang's solitons are stable when $\left(\chi_{1}+\chi_{2}\right)$ varies up to $\pm 9 \%\left(\bar{\chi}_{1}+\bar{\chi}_{2}\right)$. In the meanwhile, Pang's solitons disperse and split gradually into some small waves when $\left(\chi_{1}+\chi_{2}\right)$ are larger than this value.

With $|\beta \mathrm{n}| \leq 0.5$ for $\varepsilon==0.57 \mathrm{meV}$ and $1.26 \mathrm{meV}$, we show the behaviors of Pang's soliton in figure 24 .

These figures show that in the case of a random sequence, only if $\varepsilon<1.3 \mathrm{meV}$ and $\mid \beta \mathrm{n}$ $\mid \leq 0.5$ can Pang's soliton pass through the chain and is stable. For higher values of $\varepsilon$, Pang's soliton is reflected or dispersed.

In figure 25 we show the variations in features of Pang's solitons due to the fluctuations of $\Delta \mathrm{L}= \pm 5 \% \bar{L}$ and $\pm 6 \% \bar{L}$. These figures show that the soliton is stable only if $\Delta \mathrm{L}< \pm 7 \% \bar{L}$. From the simulation we know that the smaller the fluctuation of the chain-chain interaction, the higher the stability of Pang's soliton. When $\Delta \mathrm{L}=0$ and $\mathrm{L}=0$, the stability of Pang's soliton is the highest.

(3) Associated effects of the fluctuations of six structural parameters on the Pang's solitons.

We studied the associated influences of the change of mass of amino acids and the fluctuations in dipole-dipole interaction, the exciton-phonon coupling constant, the spring constant, the diagonal disorder, and the chain-chain interaction arising from the structure disorder of the $\alpha$-helix protein molecules, on the behaviors of Pang's soliton. 

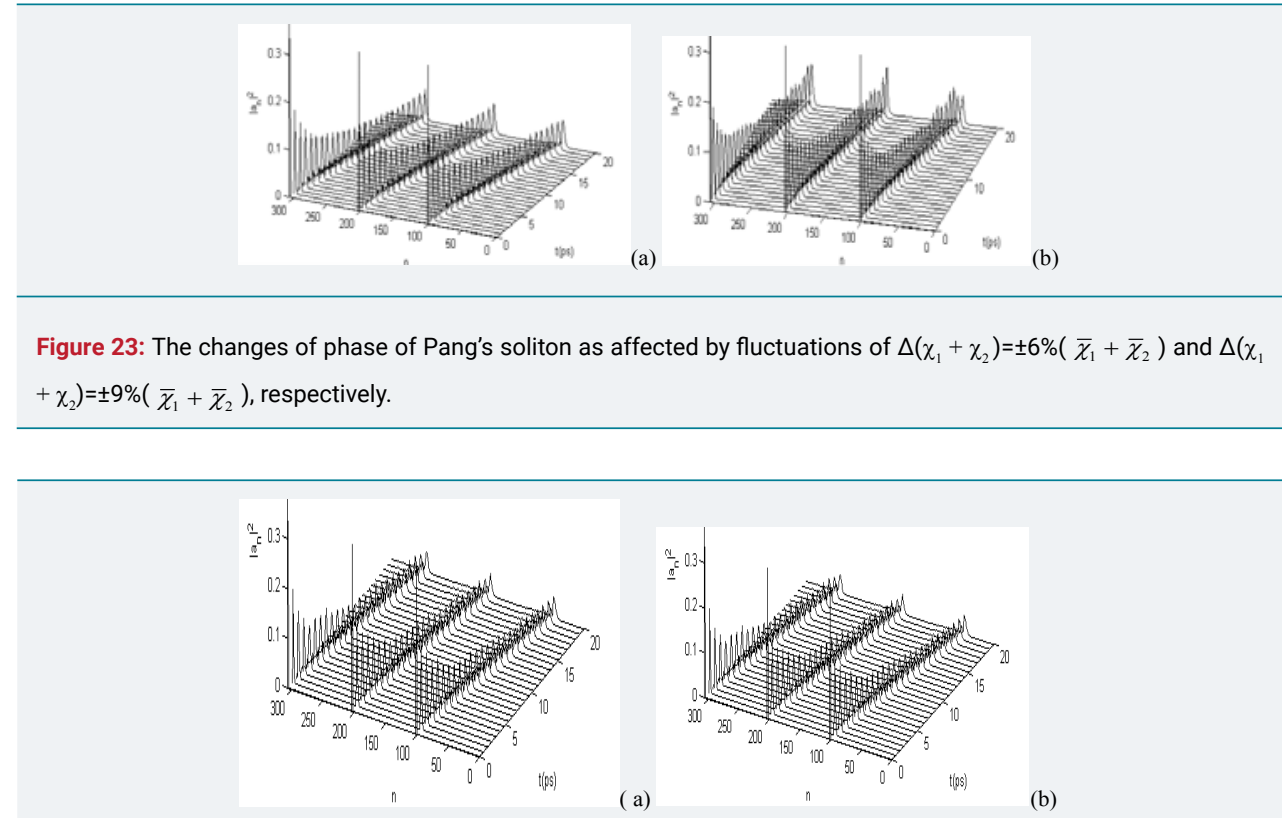

Figure 24: The behavior of Pang's solitons due to the changes $\Delta \varepsilon_{0}=\varepsilon\left|\beta_{n}\right|$ with $\left|\beta_{n}\right|<0.5$ and $\varepsilon=0.57 \mathrm{meV}(a)$ and $1.26 \mathrm{meV}(\mathrm{b})$, respectively.
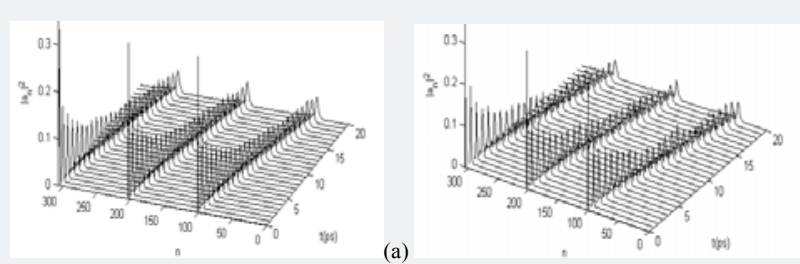

(b)

Figure 25: The variations of feature of Pang's solitons in fluctuation of $\Delta \mathrm{L}= \pm 5 \% \bar{L}$ (a) and $\pm 6 \% \bar{L}$ (b).

In figure 26 we show the changes of property of Pang's solitons under the influence of different fluctuations of structure parameters, in which figure 26a denotes the state and behaviour of Pang's soliton with fluctuations of $0.67<\alpha_{\mathrm{K}}<2, \Delta \mathrm{W}= \pm 5 \% \overline{\mathrm{W}}, \Delta \mathrm{J}= \pm 1 \%$ $\bar{J} \Delta\left(\chi_{1}+\chi_{2}\right)= \pm 1 \%\left(\bar{\chi}_{1}+\bar{\chi}_{2}\right)$ and $\Delta \mathrm{L}= \pm 1 \% \bar{L}$; figure $26 \mathrm{~b}$ results when $0.67<\alpha_{\mathrm{K}}<2$, $\Delta \mathrm{W}= \pm 10 \% \overline{\mathrm{W}}, \Delta \mathrm{J}= \pm 2 \% \bar{J} \quad \Delta\left(\chi_{1}+\chi_{2}\right)= \pm 3 \%\left(\bar{\chi}_{1}+\bar{\chi}_{2}\right)$ and $\Delta \mathrm{L}= \pm 2 \% \bar{L}$; figure $26 \mathrm{c}$ results when $0.67<\alpha_{\mathrm{K}}<2, \Delta \mathrm{W}= \pm 10 \% \overline{\mathrm{W}}, \Delta \mathrm{J}= \pm 2 \% \bar{J} \quad \Delta\left(\chi_{1}+\chi_{2}\right)= \pm 5 \%\left(\bar{\chi}_{1}+\bar{\chi}_{2}\right)$ and $\Delta \mathrm{L}= \pm 2 \% \bar{L}$ ; figure $24 \mathrm{~d}$ is the result when $0.67<\alpha_{\mathrm{K}}<2, \Delta \mathrm{W}= \pm 10 \% \overline{\mathrm{W}}, \Delta \mathrm{J}= \pm 2 \% \bar{J} \quad \Delta\left(\chi_{1}+\chi_{2}\right)= \pm 6 \%($ $\left.\bar{\chi}_{1}+\bar{\chi}_{2}\right)$ and $\Delta \mathrm{L}= \pm 2 \% \bar{L}$. From these figures we see clearly that Pang's solitons shown in figure $26 a, b$ and $c$ are very stable under the actions of different structure disorders, but Pang's soliton shown in figure $26 \mathrm{~d}$ is already unstable due to the influence of the structure disorder, its amplitude changes, thus it is already dispersed. This manifests that the solitons excited in $\Delta$-helix protein molecules are not stable in cases of large structure disorders. In comparing these results in figure 26 with those shown in figure 21-25, we find that the collective effects of these structure disorders and quantum fluctuations change the states and features of Pang's solitons, making their amplitudes, energies and velocities decrease, although such influences cannot destroy the solitons, which can still transport steadily along the molecular chains while retaining energy and momentum when the quantum fluctuations are small, i.e., Pang's the solitons are quite robust against these disorder effects. However, Pang's the solitons may be dispersed or disrupted in cases of very large structure disorders.

(4) The collective effects of various nonuniformities on the motion of Pang's soliton.

We fiwwnally study the collective effects of the change in mass of amino acids and the fluctuations of dipole-dipole interaction, the exciton-phonon coupling constant, the 

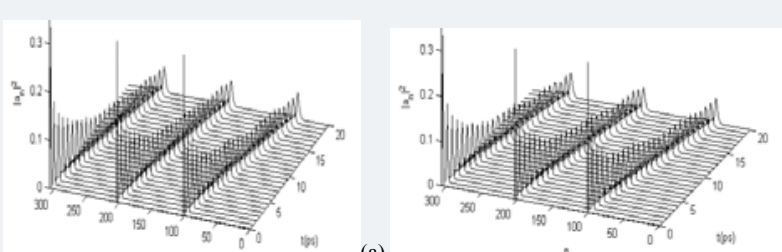

(a)

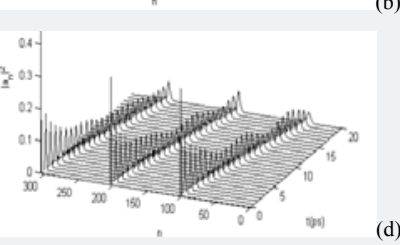

(b)

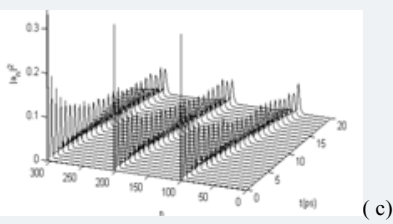

Figure 26: The changes in properties of the solitons under the influence of different fluctuations, here (a) is the result when $0.67<\mathrm{a}_{\mathrm{K}}<2, \Delta \mathrm{W}= \pm 5 \%, \Delta \mathrm{J}= \pm 1 \% \bar{J} \quad \Delta\left(\chi_{1}+\chi_{2}\right)= \pm 1 \%\left(\bar{\chi}_{1}+\bar{\chi}_{2}\right)$ and $\Delta \mathrm{L}= \pm 1 \% \bar{L}$; (b) is the result when $0.67<\mathrm{a}_{\mathrm{K}}<2, \Delta \mathrm{W}= \pm 10 \% \overline{\mathrm{W}}, \Delta\left(\chi_{1}+\chi_{2}\right)= \pm 3 \%\left(\bar{\chi}_{1}+\bar{\chi}_{2}\right), \Delta \mathrm{J}= \pm 2 \% \bar{J}$ and $\Delta \mathrm{L}= \pm 2 \% \bar{L} ;(\mathrm{c})$ is the result when $0.67<\mathrm{a}_{\mathrm{K}}<2$ $\Delta \mathrm{W}= \pm 10 \%, \Delta \mathrm{J}= \pm 2 \% \bar{J} \Delta\left(\chi_{1}+\chi_{2}\right)= \pm 5 \%\left(\bar{\chi}_{1}+\bar{\chi}_{2}\right)$ and $\Delta \mathrm{L}= \pm 2 \% \bar{L} ;$ and $(\mathrm{d})$ is the result when $0.67<\mathrm{a}_{\mathrm{K}}<2, \Delta \mathrm{W}= \pm 10 \%$ $\overline{\mathrm{W}}, \Delta \mathrm{J}= \pm 2 \% \bar{J}, \Delta\left(\chi_{1}+\chi_{2}\right)= \pm 6 \%\left(\bar{\chi}_{1}+\bar{\chi}_{2}\right)$ and $\Delta \mathrm{L}= \pm 2 \% \bar{L} . \varepsilon=0.35 \mathrm{meV},\left|\beta_{\mathrm{n}}\right|<0.5$.

spring constant, the diagonal disorder and the chain-chain interactions arising from the structure disorder of $\alpha$-helix protein molecules, on the properties of Pang's soliton. The results obtained are denoted in figure 27, in which figure 27 a represents the features of Pang's soliton formed under fluctuation conditions of $0.67<\alpha_{\mathrm{K}}<2, \Delta \mathrm{W}= \pm 3 \%$ $\overline{\mathrm{W}}, \Delta \mathrm{J}= \pm 1 \% \bar{J} \Delta\left(\chi_{1}+\chi_{2}\right)= \pm 1 \%\left(\bar{\chi}_{1}+\bar{\chi}_{2}\right), \Delta \mathrm{L}= \pm 1 \% \bar{L}$ and $\Delta \varepsilon_{0}=\varepsilon\left|\beta_{\mathrm{n}}\right|$,

$\varepsilon=0.35 \mathrm{meV},\left|\beta_{\mathrm{n}}\right|<0.5$; figure $27 \mathrm{~b}$ results when $0.67<\alpha_{\mathrm{K}}<2, \Delta \mathrm{W}= \pm 6 \% \overline{\mathrm{W}}, \Delta \mathrm{J}= \pm 1 \% \bar{J}$ $, \Delta\left(\chi_{1}+\chi_{2}\right)= \pm 2 \%\left(\bar{\chi}_{1}+\bar{\chi}_{2}\right), \Delta \mathrm{L}= \pm 1 \% \bar{L}$ and $\Delta \varepsilon_{0}=\varepsilon\left|\beta_{\mathrm{n}}\right|, \varepsilon=0.1 \mathrm{meV},\left|\beta_{\mathrm{n}}\right|<0.5$; figure $27 \mathrm{c}$ results when $0.67<\alpha_{\mathrm{K}}<2, \Delta \mathrm{W}= \pm 8 \% \overline{\mathrm{W}}, \Delta \mathrm{J}= \pm 1 \% \bar{J} \quad \Delta\left(\chi_{1}+\chi_{2}\right)= \pm 3 \%\left(\bar{\chi}_{1}+\bar{\chi}_{2}\right)$ and $\Delta \mathrm{L}= \pm 1 \% L$ and $\Delta \varepsilon_{0}=\varepsilon\left|\beta_{\mathrm{n}}\right|, \varepsilon=0.1 \mathrm{meV},\left|\beta_{\mathrm{n}}\right|<0.5$. We see from this figure that in these conditions the intrinsic nature of Pang's soliton during bio-energy transport in the $\alpha$-helix protein molecules can still be maintained, but the soliton begins to disperse when these structure disorders are larger than the values $0.67<\alpha_{\mathrm{K}}<2, \Delta \mathrm{W}= \pm 8 \% \overline{\mathrm{W}}$ ,$\Delta \mathrm{J}= \pm 1 \% \bar{J} \quad \Delta\left(\chi_{1}+\chi_{2}\right)= \pm 3 \%\left(\bar{\chi}_{1}+\bar{\chi}_{2}\right)$ and $\Delta \mathrm{L}= \pm 1 \% L$ and $\Delta \varepsilon_{0}=\varepsilon\left|\beta_{\mathrm{n}}\right|, \varepsilon=0.1 \mathrm{meV}$, $\left|\beta_{\mathrm{n}}\right|<0.5$. Therefore, we can conclude that the soliton in Pang's model is exactly robust against various structure disorders of the $\alpha$-helix protein molecules.

Hwowever, the actual degree of structure nonuniformity or disorder in protein molecules unknown up to now. The structure nonuniformity in masses of amino acid residues should smaller than the mass interval of the natural amino acid residues since the proteins are a biological self-organization with higher order, in which the amino acid residues are not free particles but are covalently bound in the main polypeptide chain. The structure nonuniformity in the other parameters should be small due to small influences of the side groups on the geometry of the main chain and disorder distribution of the mass. Thus we can conclude that the naturally occurring the structure nonuniformity in the parameters should be smaller than the maximal structure nonuniformity in which the Pang's soliton, is stable. Natural structure nonuniformity may interfere with the solitons only when J and $\varepsilon$ are obviously varied since the stability interval in these cases is smaller relative to other parameters. However, the fluctuations of $J$ and $\varepsilon$ for Pang's soliton are also larger as mentioned above. Thus it is not necessary to doubt that the structure nonuniformity or disorder in protein molecule can destroy the states of soliton in Pang's model, thus the soliton transport of bio-energy can still maintain although the influences of natural structure nonuniformity or disorder on it occur in biological protein molecules.

The influences of temperature of systems on the energy transport in protein molecules 


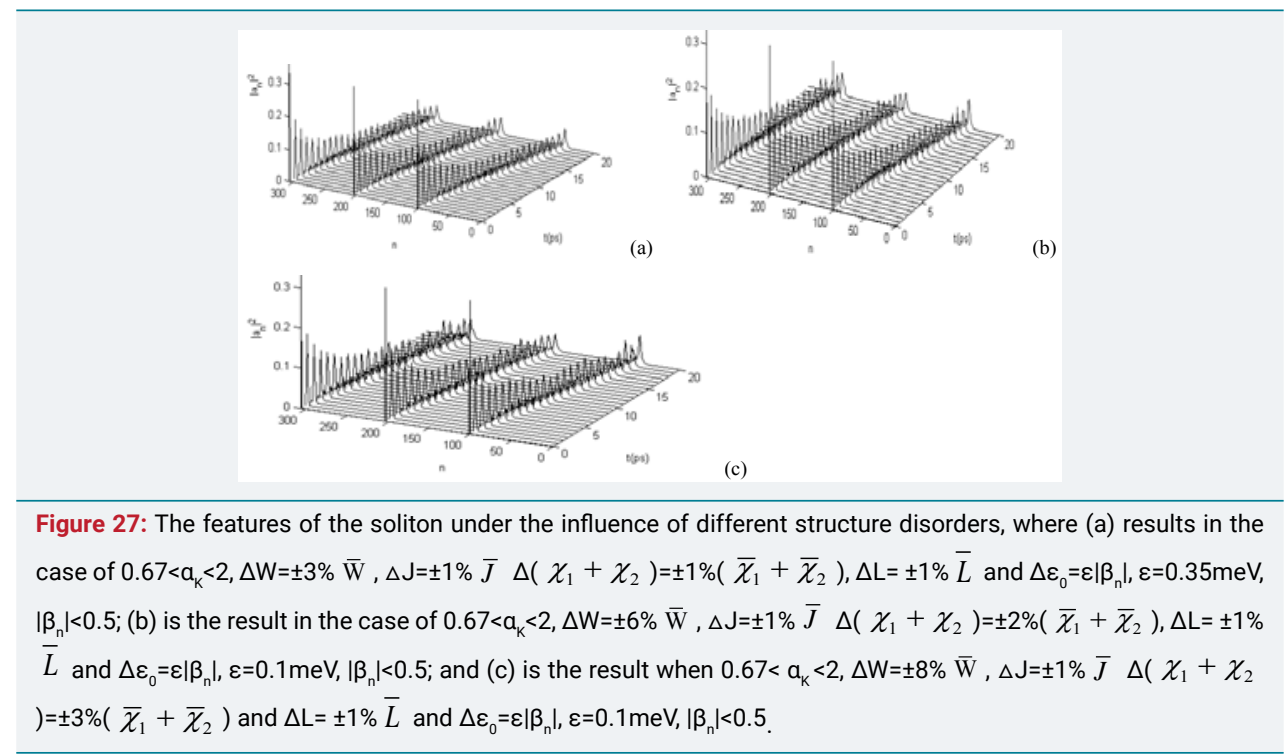

As it is known, the thermal stability and lifetime of the solitons, which transport the bio-energy in protein molecules, at the physiological temperature $300 \mathrm{~K}$ are a key indicator to inspect and check the validity of the theory of bio-energy transport and true existence of solitons. Therefore, plenty of researchers study this problem using different methods. The following statements are a simple survey on these investigations.

Investigations on thermal stability of the Davydov solitons in range of physiological temperature in uniform protein molecules

First of all, Davydov investigated [153] the influences of thermal perturbation at $300 \mathrm{~K}$ on the soliton through the thermodynamic average of Hamiltonian of the protein molecules, in which he thought that the thermal perturbation results in the thermal excitation of the phonons in this system. Thus the Davydov's wave function, $\left|D_{1}\right\rangle$, in Eq.(2) was replaced by $\left.\left|\bar{D}_{1}\right\rangle=|\varphi(t)>| \beta(t)\right\rangle|v\rangle$, where $|v\rangle=\left|\left\{v_{q}\right\}\right\rangle=\prod_{q}\left|v_{q}\right\rangle=\prod_{q}\left(v_{q} !\right)^{1 / 2}\left(a_{q}^{+}\right)^{v_{q}}|0\rangle_{p h}$. If the Hamiltonian of the system in Eq.(1) in Ref.[1].is represented by the creation and annihilation operators of the exciton and phonon, Davydov denoted the thermodynamic average of the Hamiltonian by $H^{\prime}=\sum_{v} \rho_{v v} H_{v v}$, where $H_{v v}=\left\langle\varphi\left|\left(H_{e x}+H_{\text {int }}\right)\right| \varphi\right\rangle+\left\langle v\left|U^{*} H_{p h} U\right| v\right\rangle$ with and $|\beta(t)\rangle=U_{n}|0\rangle_{p h}=$ $\exp \left\{\sum_{q}\left[\beta_{n q}(t) a_{q}^{+}-\beta_{n q}^{*}(t) a_{q}\right]\right\}|0\rangle_{p h}$. Utilizing familiar method Davydov obtained the dynamic equation of the soliton in this case. From this dynamic equation Davydov obtained that the effective mass and nonlinear interaction energy depend on the temperature of the system, i.e., with increasing temperature the effective mass of Davydov soliton increases and the nonlinear interaction energy decreases, the soliton's size increases and its properties come ever closer to these of an exciton. Meanwhile he obtained also the dependence of the soliton parameters on its velocity. When $\mathrm{v}<\mathrm{v}_{\mathrm{o}}$ and $\mathrm{v}$ approaches $\mathrm{v}_{0}\left(\mathrm{v}_{0}\right.$ is the sound speed of protein molecules), the nonlinear interaction energy increases. At sufficiently high temperatures, a loss of localization of Davydov soliton occurs. In this case the soliton energy increases and its size decreases.

Subsequently, L. Cruzeiro et al. [154,] used also the thermodynamic average Hamiltonian $H_{T}=\sum_{v} \rho_{v v} H_{v v}$ mentioned above, but $H_{v v}=\left\langle\bar{D}_{l}\right|\left(H_{e x}+H_{\text {itt }}+H_{p h}\left|\bar{D}_{\mathrm{l}}\right\rangle\right.$. From again $i \hbar \frac{\partial \varphi_{n}}{\partial t}=\frac{\partial H_{T}}{\partial \varphi_{n}^{-}}$and $i \hbar \frac{\partial \beta_{n}}{\partial t}=\frac{\partial H_{T}}{\partial \beta_{n q}^{*}}$ they obtained the dynamic equations of $\varphi_{n}$ and $\beta_{n q}$, respectively, where $\beta_{n q}^{*}+\beta_{-q n}=-\left\langle v\left|U_{n}^{+}\left(a_{q}^{+}+a_{-q}\right) U_{n}\right| v\right\rangle$, which are all the nonlinear Schrodinger equations. They found further out the solutions of the two equations using numerical 
simulation method. The results obtained for the probability of an soliton excitation $\left|\varphi_{n}\right|^{2}$ at physiological temperature $310 \mathrm{~K}$ are shown in figure 28 , where $M=114 \mathrm{~m}_{\mathrm{p}}, \mathrm{J}=7.8 \mathrm{~cm}^{-1}$ and $\mathrm{w}=13 \mathrm{~N} / \mathrm{m}$ are used, but $\varepsilon_{0}=1666 \mathrm{~cm}^{-1}$ is removed from the numerical calculations through a gauge transformation, the initial condition used are $\left|\varphi_{24}(0)\right|^{2}=\left|\varphi_{24}(0)\right|^{2}=0.5$ and $\beta_{n q}(0)=0$. Figure 28(a), (b) and (c) are the results of $\chi_{1}=0.17 \times 10^{-10} \mathrm{~N}, 0.21 \times 10^{-10} \mathrm{~N}$ and $0.23 \times 10^{-10} \mathrm{~N}$, respectively.

Figure 28(a) shows a case in which the initial excitation is completely dispersed after 10 psec.

Indeed, only dispersive waves, which travel at about $1 / 3$ the maximum speed of sound in the chain $\left[v_{0}=r_{0} \sqrt{\mathrm{w} / \mathrm{M}}=3.7 \times 10^{3} \mathrm{~m} / \mathrm{sec}\right]$ is formed and is not accompanied by any molecular displacement. They are therefore excitons. Excitons are generated which travel in opposite

directions in the chain and interfere with each other 10 psec afterwards. This interference is an artificial phenomenon due to the periodic boundary conditions. Figure 28(b) shows another situation in which part of the initial excitation is not dispersed and remains pinned in the same bonds where it was initially located. Finally, in figure 28(c), where most of the initial excitation is not dispersed but remains localized where it was put initially. This result shows that the continuous transition from dispersion (figure 28(a) to a localized state (figure 28(c)) of the initial excitation is observed at biological temperatures $310 \mathrm{~K}$. In this case, the consequence of increasing temperature is similar to that of increasing the nonlinearity, i.e., as temperature increases, the threshold for localization of the initial excitation decreases. Increasing the temperature thus produces a decrease in the effective dispersion, as was pointed out by Davydov [153]. Thus they obtained that the Davydov soliton is stable at $310 \mathrm{~K}$.

Cruzeiro-Hansson et al[42,45,155]studied also the influence of temperature of the system on the states of Davydov soliton. Their method is to determine an appropriate feature of the system, such as localization, number probability $\left|\varphi_{n}(t)\right|^{2}$ and amino acid's displacement $\left(u_{N / 2}-u_{N / 2-1}\right)$, to select an appropriate observable $\mathrm{Q}$ capable of describing the feature, and to compute from Gibbs statistical-mechanics prescription the equilibrium average value of $Q$ at physiological temperature region by $\left\langle<Q>>=\iint d y d \pi \operatorname{Tr}\left[Q \exp \left(-H / K_{B} T\right)\right] / \iint d y d \pi \operatorname{Tr}\left[\exp \left(-H / K_{B} T\right)\right]\right.$, where $K_{B}$ is the Boltzmann constant. The integrations are over all the vibrational coordinates and momenta, and the trace is over the quasiparticle states. Their results show that thermal destabilization is not associated with transitions to exciton states and that it involves instead disordered states with energies intermediate between these of the minimum
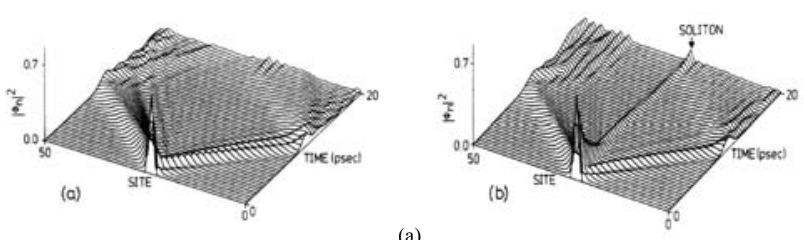

(b)

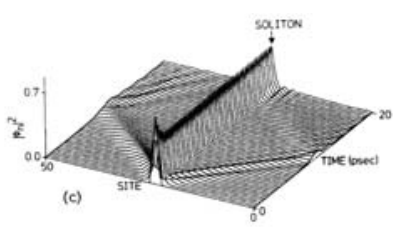

(c)

Figure 28: The evolution of the probability of Davydov soliton in the time and space at $310 \mathrm{~K}$ (see Cruzeiro et al, paper [45]). 
energy soliton state and those of the exciton states. A basic duality emerges regarding the effect of temperature on the stability of nonlinear structures: temperature is found to help the nonlinearity in certain parameter and temperature regimes by inducing disorder and to destroy the nonlinearity in other regimes, e.g., always at large temperatures as a consequence of Boltzmann equalization. Thus they obtained that the Davydov system exhibits " universal" behavior at physiological temperatures.

In the meanwhile, Bolterauer $[72,156]$ argued that their classical thermalization scheme should lead to incorrect results when applied to a quantum system. Bolterauer and other workers found the Davydov solitons to be stable at $\mathrm{T}=300 \mathrm{~K}$.

As it is known, Davydov's model yields indeed a compelling picture for the mechanism of bio-energy transport in protein molecules, but there are considerable controversy concerning whether the Davydov soliton is thermal stable at $300 \mathrm{~K}$ and can provide a viable explanation for bio-energy transport. It is out of question that the thermal perturbations are expected to cause the Davydov soliton to decay into a delocalized state. The above simulations showed that the Davydov soliton is stable at $300 \mathrm{~K}$ [153-154], but they were based on classical equations of motion which are likely to yield unreliable estimates for the stability of the Davydov's soliton. Since the dynamical equations used in the simulations are not equivalent to the Schrödinger equation, the stability of the soliton obtained by these numerical simulations is unavailable or unreliable.

Lawrence et al. [69,157], proposed a model incorporating exciton-phonon interaction as a mechanism for localizing and stabilizing energy transport in the longchain proteins. This is due to the fact that previous analytical and numerical studies $[9-12,15,16,153,154]$ have not adequately addressed the effects of thermal phonons, which may act to disperse exciton energy. Thus they finished a numerical calculation, in which they indicated that the excitations are strongly dispersed at physiological temperament. They think that the propagation of the exciton-phonon state at low temperatures makes a transition from a solitary wave mode to a stationary self-trapped mode as the coupling between excitons and phonons is increased. Thus they obtained a new result of calculations of exciton-normal-mode coupling in the formamide dimer, which indicate that more sophisticated models are necessary to yield the true coupling constant in proteins. Thus they calculated a parametric study of the Davydov model of energy transport in alpha-helical proteins. Previous investigations have shown that the Davydov model predicts that nonlinear interactions between phonons and amide-I excitations can stabilize the latter and produce a long-lived Davydov soliton, which propagates along the alpha-helix proteins. The dynamics of this solitary wave are approximately those of solitons described using the nonlinear Schr6dinger equation. However, the present study based on the new calculation extends these previous investigations by analyzing the effect of helix length and nonlinear coupling efficiency on the phonon spectrum in short and medium length alpha-helical segments. The phonon energy accompanying amide-I excitation shows periodic variation in time with fluctuations that follow three different time scales. The phonon spectrum is highly dependent upon chain length but a majority of the energy remains localized in normal mode vibrations even in the long chain alpha-helices. Variation of the phonon-exciton coupling coefficient changes the amplitudes but not the frequencies of the phonon spectrum. The computed spectra contain frequencies ranging from $200 \mathrm{GHz}$ to $6 \mathrm{THz}$, and as the chain length is increased, the long period oscillations increase in amplitude. The most important prediction of their study, however, is that the dynamics predicted by the numerical calculations have more in common with dynamics described by using the Frohlich polaron model than by using the Davydov soliton. Thus, they finally concluded that the relevance of the Davydov soliton mode, which was applied to energy transport in alpha-helical proteins, is questionable. 
Lomdahl et al. [158], investigated the temperature effect on the Davydov soliton through adding the damping force and noise force, $f_{n}=-m \Gamma \dot{u}_{n}+F_{n}(t)$, into the discrete dynamic equation (20)of displacement of amino acids, $u_{n}(t)$, and let $L=\alpha=0$ in Eq.(19). In accordance with statistical physics, the thermal noise term $F_{n}(t)$ is related with the temperature of the systems, its correlation function can be represented by $<F(x, t) F(0,0) \geqslant 2 M \Gamma K_{B} T \delta(x) \delta(t) / \tau^{\prime}$, where $\tau^{\prime}$ is the damping constant, $\mathrm{T}$ is the temperature of the system. It is assumed that the random deviation of the noise obey the normal distribution with criterion deviation $\sqrt{\sigma}$ and a zero expectation value. That is,

$$
N\left(F_{n}\right)=\frac{1}{\sqrt{2 \pi \sigma}} \exp \left[-F_{n}^{2} / 2 \sigma\right],
$$

where $\sigma=2 \mathrm{MK}_{\mathrm{B}} \mathrm{T \Gamma} / \tau^{\prime}, \tau^{\prime}$ is the time constant, and $\Gamma$ is the reciprocal time constant of the heat bath. It can be shown that $F_{n}(t)=\sqrt{\sigma} \sum_{\sum}^{L}\left[X_{n}(t)-\frac{1}{2}\right]$, where $X_{\text {nr }}(t)$ is a random number between 0 and 1. If we choose $\mathrm{L}=12$, then the deviation of $\left[x_{u}(t)-\frac{1}{2}\right]$ is $1 / 12$, and the standard deviation of $\mathrm{F}_{\mathrm{n}}(\mathrm{t})$ is $\sqrt{\sigma}$. The domain of the random noise force is just $\left|F_{n}(t)\right| \leq 6 \sqrt{\sigma}$.

In the meanwhile, they verified numerically to high accuracy that over sufficiently long time intervals this gives for the mean kinetic energy $\left\langle\sum_{n}^{1} \frac{1}{2} \mathrm{mu}_{n}^{2}(t)\right\rangle=\frac{1}{2} N K_{B} T$.

They used these equations to study the properties of solution of the dynamic equations by the soliton detector. To check the consistency of the result, the calculations were also done in the conventional microcanonical ensemble. The system was prepared at $T=300 \mathrm{~K}$, it was then allowed to evolve only under the influence of the dynamic equations. The result of these simulations shows that the Davydov soliton is instable at $300 \mathrm{~K}$ and disappear in a few picoseconds as shown in figure 29, which is similar with the results obtained by Lawrence et al. [69,157]. Lomdahl et al. [158], give further the states and features of Davydov soliton containing $Q^{\prime}=\left\langle D_{2}\left|\sum_{n} B_{n}^{+} B_{n}\right| D_{2}\right\rangle=2$ and 6 quanta under influences of damping force and thermal noise at $\mathrm{T}=300 \mathrm{~K}$ using the above method, which are shown in figures 29,30 , respectively. These results indicate clearly that the Davydov soliton formed in these conditions cannot still be thermally stable at biological temperature $300 \mathrm{~K}$, whether it contains one quantum or two or six quanta.

On the other hand, Förner's [150] used the above Lomhahl et al's method, formulae and the fourth-order Runge-Kutta method [147-148] to calculate the changes of stability of the Davydov soliton with varying temperature of the system. They find that the Davydov soliton is only thermally stable at $\mathrm{T}<40 \mathrm{~K}$, but begins to disperse at $40 \mathrm{~K}$, and is destroyed completely at $300 \mathrm{~K}$, as shown in figures $31-33$. Therefore the Davydov soliton is not thermally stable at $300 \mathrm{~K}$; its critical temperature is only $40 \mathrm{~K}$. These are consistent with the analytic results [105-107].

Wang et al. [64], studied the effects of thermal perturbation on properties

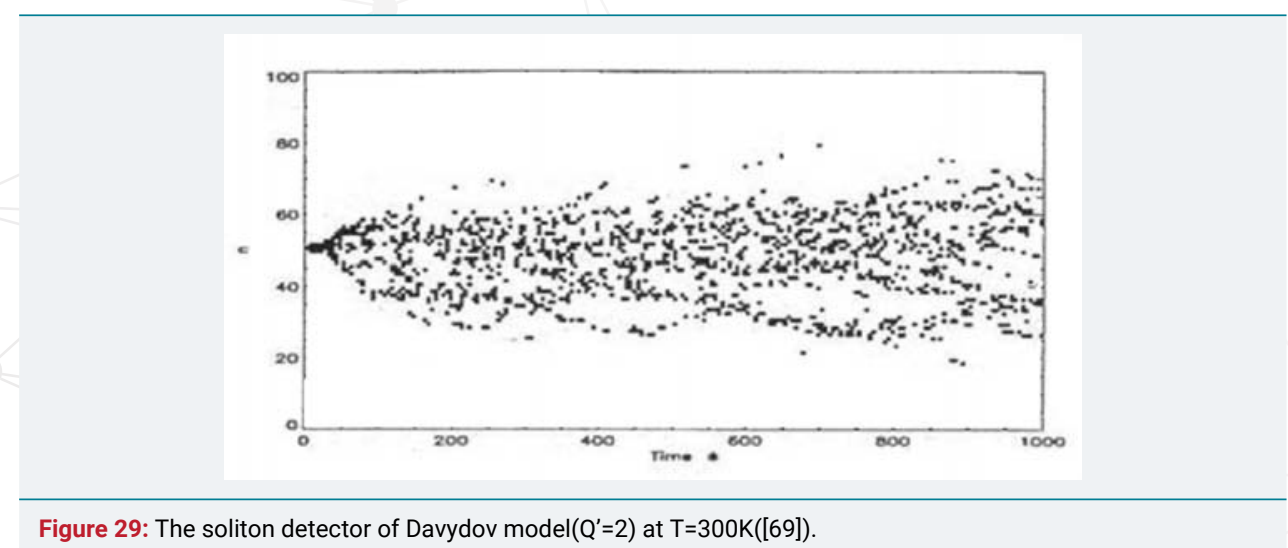

Figure 29: The soliton detector of Davydov model( $\left.Q^{\prime}=2\right)$ at $T=300 K([69])$. 

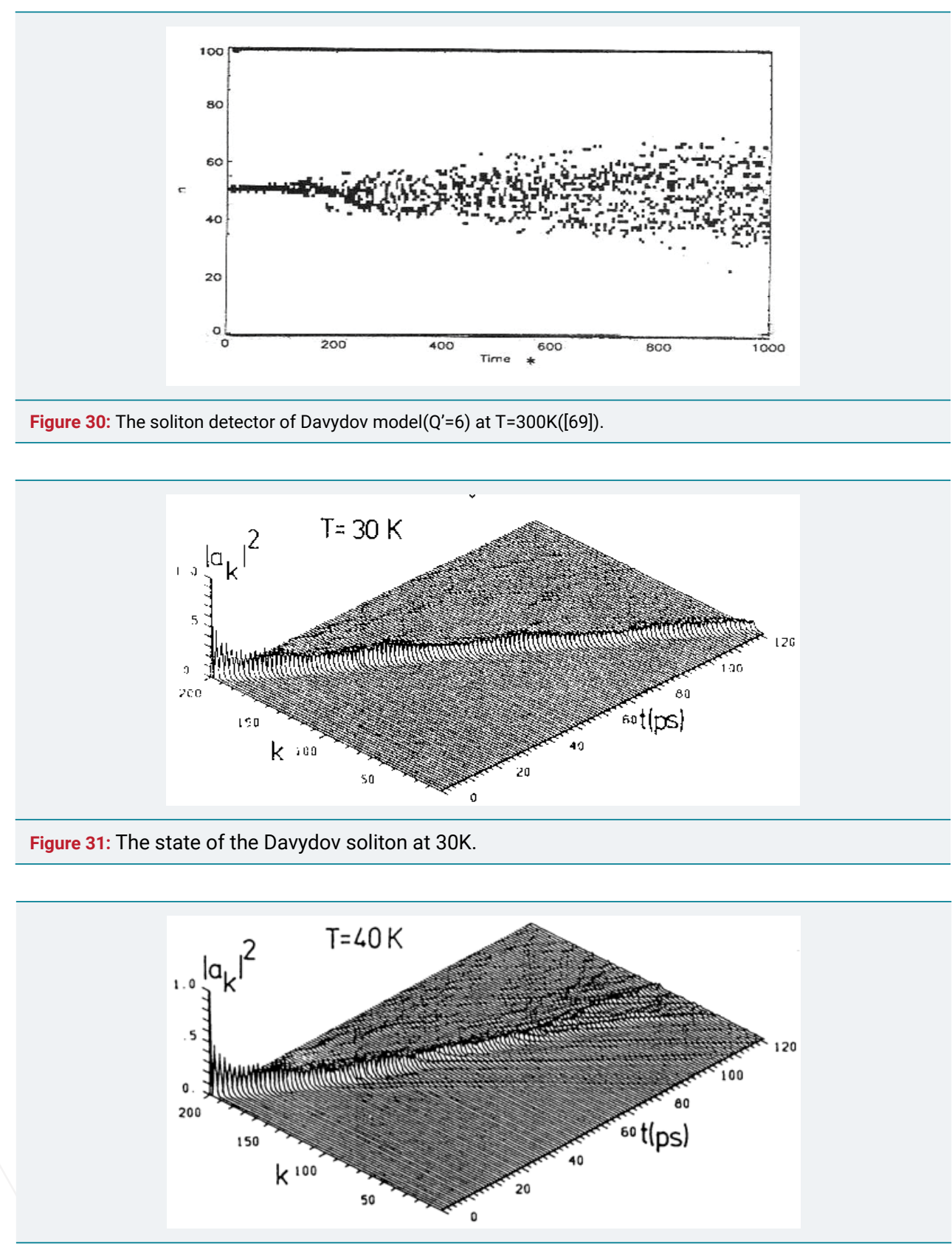

Figure 32: The state of Davydov soliton at 40K (See Ref.[150]) (See Ref.[150]).

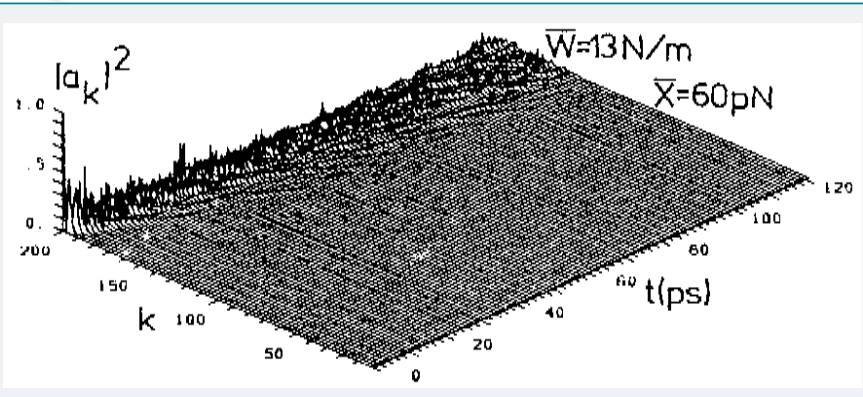

Figure 33: The state of Davydov soliton at300K[150]

of Davydov soliton by quantum Monte Carlo approach. In this case, the thermal equilibrium expectation value of any physical observable $Q$ in the canonical ensemble is give $\langle\langle Q\rangle>=\operatorname{Tr}[Q \exp (-\beta H)] / \operatorname{Tr}[\exp (-\beta H)$, which is formulated by Feynman's path integral for the quantum propagator with imaginary time $\beta$. The partition 
function $Z=\operatorname{Tr} \exp (-\beta H)$ ] can be calculated by first dividing the imaginary time $\beta$ into L intervals $\Delta \tau=\beta / L$. Between each pair of intervals they insert a complete set of basis states, representing all L-polygonal arcs through the Hilbert space connecting the initial and final imaginary times. The basis states are products of exciton number states and the position eigenstates of the lattice. Using the checkerboard decomposition technique, they separated the Davydov Hamiltonian in Eq.(1) into two parts, $\mathrm{H}=\mathrm{H}_{2}\left[\mathrm{P}_{\mathrm{n}}\right]+\mathrm{H}_{1}\left[\mathrm{u}_{\mathrm{n}}\right]$, which $P_{n}=M \dot{u}_{n}, H\left(P_{n}\right)$ contains all lattice momentum operators $\mathrm{P}$ and all exciton hopping terms from even-numbered sites, and $H\left(u_{n}\right)$ contains all lattice position operators together with all exciton hopping terms from odd-numbered sites. Thus they found out.

$$
\begin{aligned}
& z \approx \int \prod_{n i} d u_{n i} \exp \left\{-\Delta \tau \sum_{n i}\left[\frac{M}{2}\left(\frac{u_{n i+1}-u_{n i}}{\Delta \tau}\right)^{2}+\frac{w}{2}\left(u_{n+1 i}-u_{n i}\right)^{2}\right]\right\} \operatorname{Tr} \coprod_{n=1}^{L} \exp \left[+\Delta \tau J \sum_{n \text { even }}\left(B_{n+1}^{+} B_{n}+\right.\right. \\
& \left.\left.\left.\left.B_{n}^{+} B_{n+1}\right)\right] \times \exp \left[-\Delta \tau \sum_{\text {nodd }}\left\{\chi_{1}\left[\left(u_{n+1 i}-u_{n-1 i}\right) B_{n}^{+} B_{n}+\left(u_{n+2 i}-u_{n i}\right) B_{n+1}^{+} B_{n+1}\right)\right]-J\left(B_{n+1}^{+} B_{n}+B_{n}^{+} B_{n+1}\right)\right\}\right]\right]^{\prime}
\end{aligned}
$$

Where in $\mathrm{u}_{\mathrm{ni}}$ is the position of the nth lattice mass at the ith cut. The above formula is just the basis of quantum Monte Carlo simulation. To utilize this formula Wang et al gained the following conclusions: (1) A coherent structure exists for temperatures below $7 \mathrm{~K}$; (2) the basic unit of this coherent structure is highly localized and bears a close resemblance to the Davydov soliton if discreteness corrections to the latter are taken into account; and (3) above $7 \mathrm{~K}$ thermal perturbation are effective in destroying the internal coherence of this basic unit, its destruction being essentially complete above $11.2 \mathrm{~K}$. These results are largely consistent with the above dynamical simulations of Lomdahl and Kerr based on $\left|D_{2}\right\rangle$ ansatz states and Cottingham et al.'s straightforward quantum-mechanical perturbation calculation [66], in which the lifetime of the Davydov soliton obtained by using this method is too small (about $10^{-12}$ $10^{-13}$ ) to be useful in biological processes. A major difference is that their method gave quantum fluctuations, the equilibrium quantity is not seriously affected by quantum fluctuations, but it is likely that dynamical properties would be affected by the presence of intrinsic quantum noise.

At the same time, Förner[150] studied further the behaviors of the Davydov soliton using the

perturbation method, in which he diagonalize the Hamiltonian partially by Cottingham et al., way [66]. Thus the Hamiltonian of the systems is denoted as $\mathrm{H}=\mathrm{H}_{0}$ $+\mathrm{V}$, here $\mathrm{H}_{0}$ is the diagonal part of $\mathrm{H}$ and $\mathrm{V}$ the non-diagonal part. Thus he treated $\mathrm{V}$ as a perturbation and thought that $\left|D_{2}\right\rangle$ is an exact eigenstate of $\mathrm{H}_{0}$ and calculated further the lifetime of Davydov soliton by first-order perturbation theory in a cyclic chain of 201 units using the symmetric interaction, where only pinned soliton were found numerically, in which $\chi_{1}=62 \mathrm{pN}, \mathrm{w}=13 \mathrm{~N} / \mathrm{m}, \mathrm{M}=114 \mathrm{~m}_{\mathrm{p}}, \mathrm{J}=0.967 \mathrm{meV}$, and $\varphi_{n}(t=9)=A \sec h\left[(n-100) \chi_{1} / w J\right]$ as the initial conditions are used. In Figure (34), exhibited the states and changes of the Davydov soliton and $w(t)=\left|\left\langle D_{2} / \Phi\right\rangle\right|$ at $0.2 \mathrm{meV}$ as the time changes at $300 \mathrm{~K}$, where $\left\langle D_{2} / \Phi\right\rangle=\exp (i \Delta t / \hbar) A \sec h\left[\left(n-n_{0}\right) \chi_{1}^{2} / w J\right.$.These results indicate clearly that the Davydov soliton is still unstable at $300 \mathrm{~K}$.

Summarily, we cannot demonstrate still that the Davydove soliton is thermally stable, although many different techniques and methods of thermal perturbation of environment were used. Therefore we can conclude from the above investigations that the Davydov soliton is unstable at physiological temperature $300 \mathrm{~K}$, thus it cannot play an important role in biological processes of bio-energy transport in the protein molecules.

The influences of temperature of proteins on bio-energy transport in Pang's model

The results in a single chain: Since protein molecules work always at a biological temperature of $300 \mathrm{~K}$, we should firstly decide whether or not the thermal motion of the 

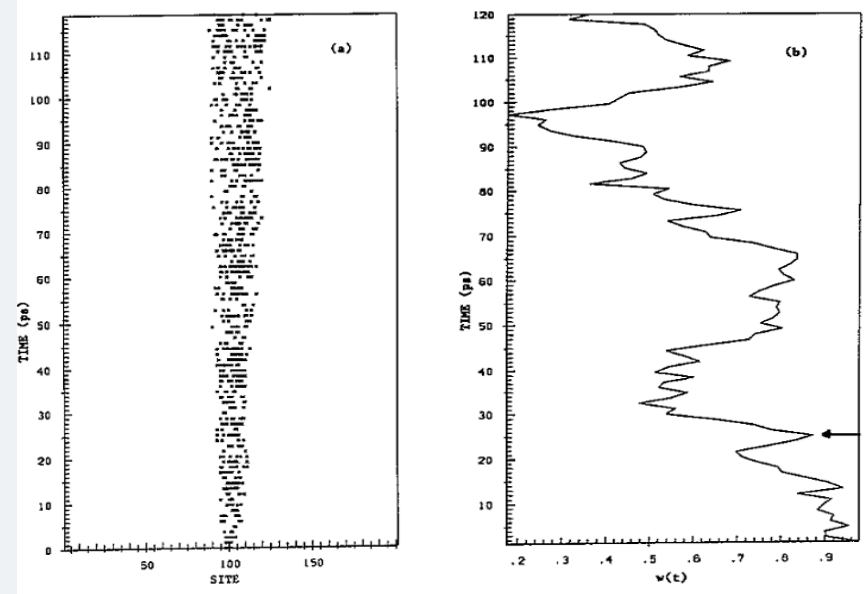

Figure 34: The Davydov soliton detector plot at $300 \mathrm{~K}$ (a) and Time evolution of $w(t)$ at $\mathrm{J}=0.2 \mathrm{meV}(\mathrm{b})$ ([150]).

lattice still permits soliton motion in such a case. In accordance with thermodynamic theory, from a $\mathrm{C}=0$ vibrational energy of $\varepsilon_{0}=3.28 \times 10^{-20} \mathrm{Nm}$ and a temperature of $300 \mathrm{~K}$, one finds that the Boltzmann factor is $3 \times 10^{-4}$ in this case. This means that only three out of 10,000 excitons are excited in the thermal equilibrium at $300 \mathrm{~K}$. Then one can safely assume that the temperature of medium primarily affects soliton motion via only the lattice of the amino acid residue. Thus, prior to soliton commencement the system is in equilibrium with the temperature of environment and the lattice is in thermal motion (which can be described as a linear combination of its normal modes), while the exciton system is in its ground state. With soliton commencement a nonequilibrium state is created, the state of the lattice changes, and the behavior of the soliton is influenced by the thermal perturbation via the lattice. This amounts to assume that the time the soliton needs to travel through the protein is small compared to the time the temperature of environment needs to re-establish equilibrium with the system due to high velocity of the soliton. However, how do we represent the effect of the heat bath on the lattice? Here we [113-125] adopt the above Lomdahl and Kerr's $[64,158]$ and Lawrence and co-worker's $[69,157]$ method, which found that the Davydov soliton is destroyed at $300 \mathrm{~K}$ by using its dynamic equations, to study the influences of the temperature of the systems on bio-energy transport in Pang's model. Thus we added the decay term $M \Gamma \dot{u}_{n}$ and random noise term, $\mathrm{F}_{\mathrm{n}}(\mathrm{t})$, resulting from the temperature and damping of medium, into the displacement equation of the amino acid molecules, in Eq. (27), where $M \Gamma \dot{u}_{n}$ and $\mathrm{F}_{\mathrm{n}}(\mathrm{t})$ are decided by the above formulae, $\Gamma$ is a dissipation coefficient for the vibration of amino acids, which is about $10^{8} \mathrm{~s}^{-1}$ for the proteins. Thus we can study the influences of dissipation force and random noise forces, arising from the heat bath, on the states of Pang's soliton by the fourth-order Runge-Kutta method [147-148] .

When the soliton starts at $t=0$, the initial condition of $a_{n}(0)=A \operatorname{sech}\left[\left(n-n_{0}\right)\right.$ $\left.\left(\bar{\chi}_{1}+\bar{\chi}_{2}\right)^{2} / 4 \bar{J}_{\mathrm{W}}\right]$ is added in one end of the protein molecule, the lattice energy fluctuations associated with the environment are larger by roughly three orders of magnitude than the local lattice energies associated with the soliton motion, but the soliton can still moves through the chain completely undisturbed at the biological temperature $(300 \mathrm{~K})$ as shown in figure 35 , where the above average values of the physical parameters of proteins were used. Therefore, despite the large lattice-energy fluctuations due to the heat bath, the nonlinear interaction between the lattice and the amide oscillators (excitons) is still able to stabilize the soliton, or in other words, the thermal perturbation at $300 \mathrm{~K}$ cannot destroy Pang's soliton. The state of the soliton in the case of a long time period (300ps) at $300 \mathrm{~K}$ and a higher temperature of 310 $\mathrm{K}$ as shown in figures 36,37 , respectively. These results [113-125] show clearly that 
the soliton in Pang's model is thermally stable in the region between $300 \mathrm{~K}$ to $310 \mathrm{~K}$. The lifetime of Pang's soliton is at least about 300ps at 300K. This means that Pang's soliton has a long enough lifetime enabling it to play an important role in biological processes. These results agree with analytic data in table In Eq.(19). However, at the high temperatures of $320 \mathrm{~K}$ and $325 \mathrm{~K}$, Pang's soliton disperses gradually as shown in figure 38,39, respectively. Thus the critical temperature of Pang's soliton is about 325K. Therefore Pang's soliton is thermally stable at 300K.These results are evidently different from those of Davydov soliton in figures 31-33 obtained by Förner [150-152].

The results in $\alpha$-helix protein molecules: For the $\alpha$-helix protein molecules the influences of biological temperature on the bio-energy transport can be studied in accordance with the above method, in which we still add the decay term $M \Gamma \dot{q}_{n}$ and random noise term, $F_{n}(t)$, resulting from the temperature into the displacement equation of the amino acid molecules in Eq.(31) according to the above way and
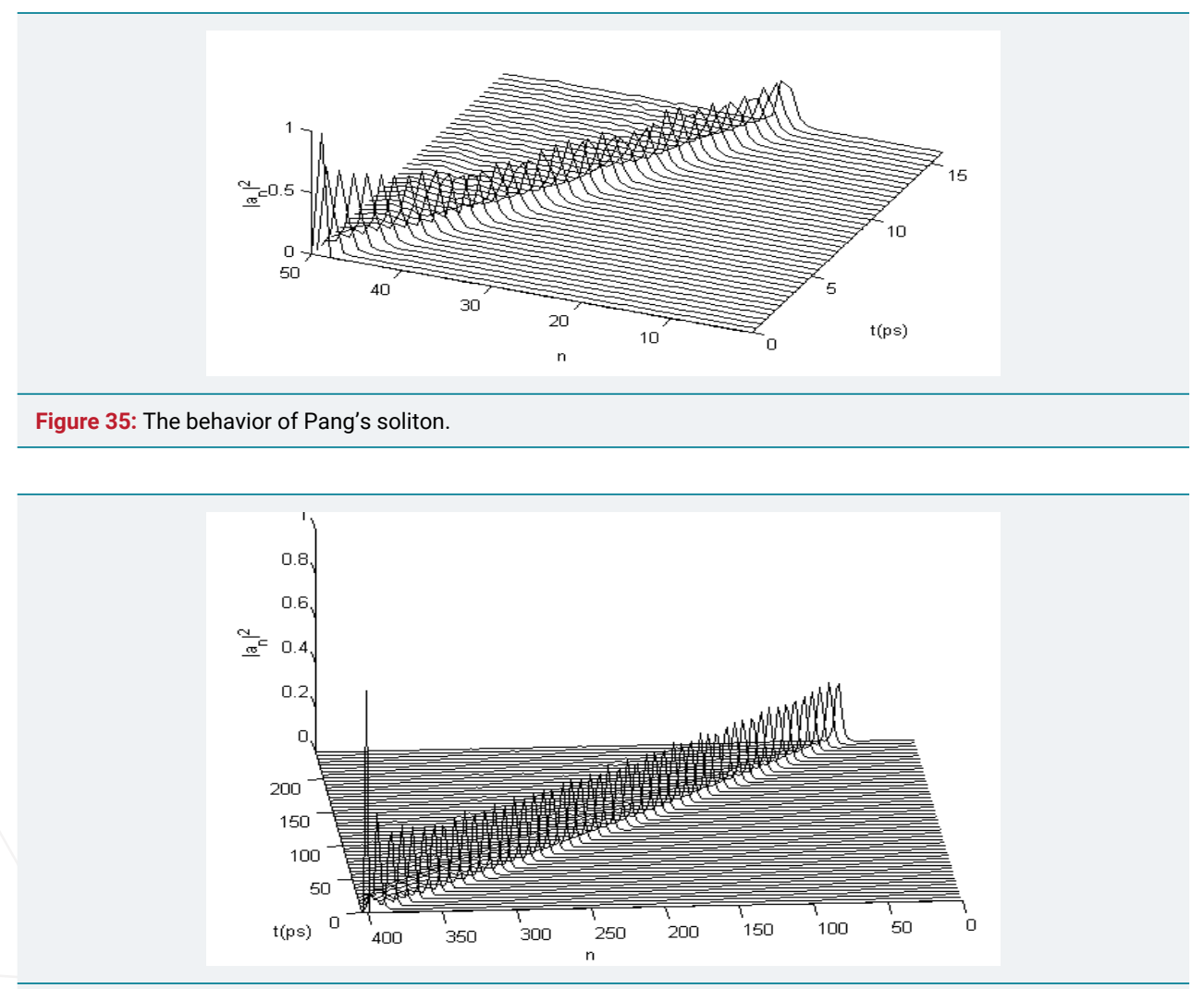

Figure 36: The state of Pang's soliton through at 300K the time period of 300ps at 310K.

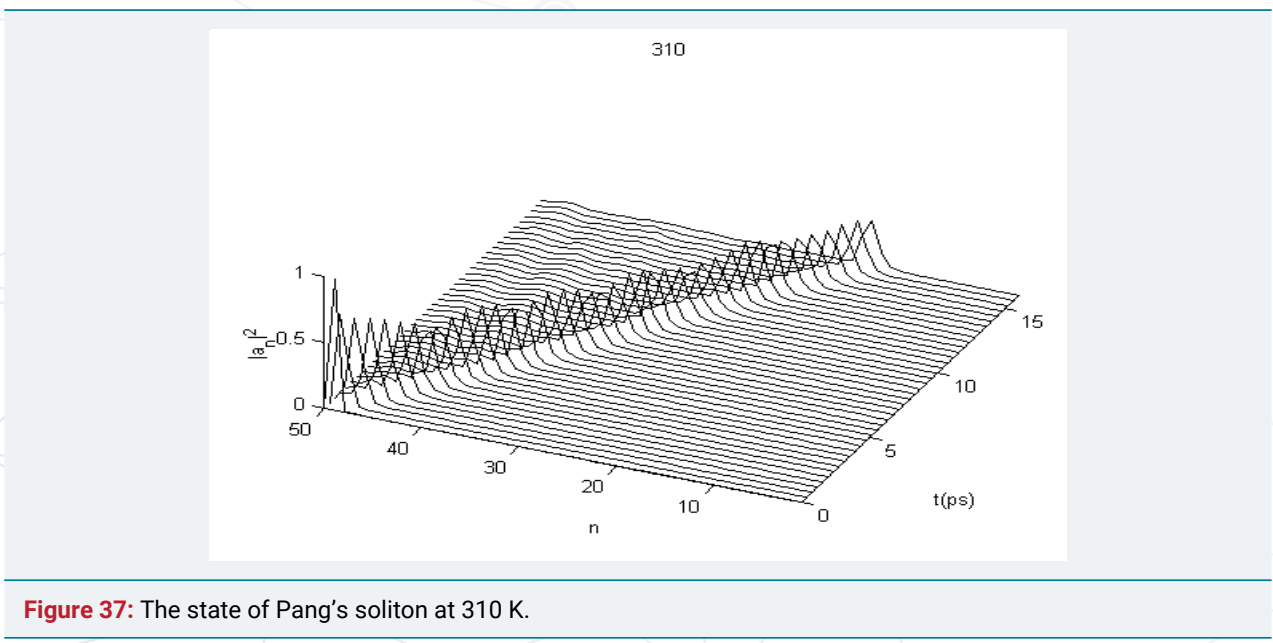



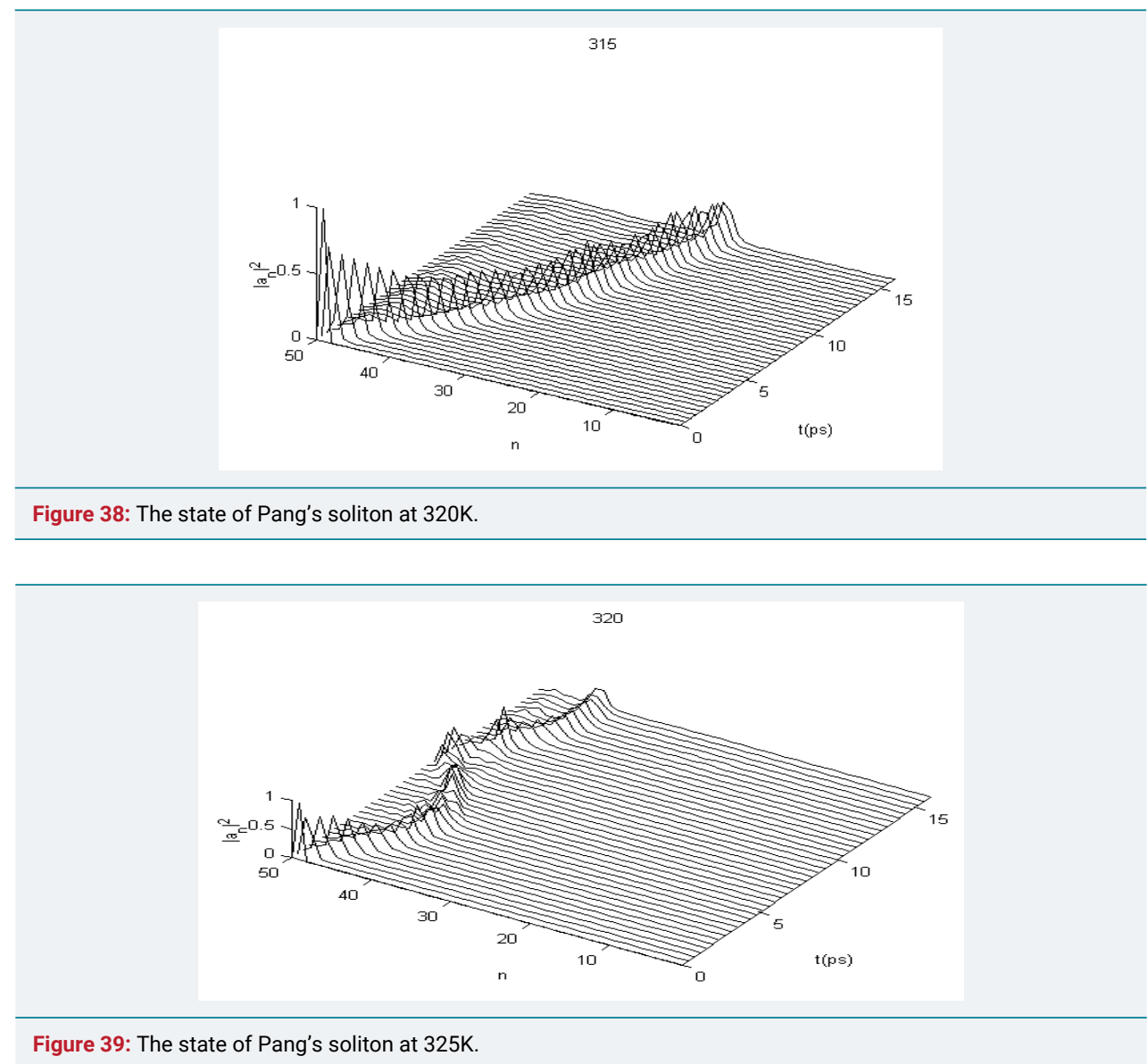

Lomdahl and Kerr's method[64,158]. We found the soliton solution of the equations of motions in Eqs.(30) and (31) with decay effect and random noise force using the above method and the fourth-order Runge-Kutta method[147-148]. The results at $300 \mathrm{~K}$ are shown in figure 40 for the $\alpha$-helix protein molecules with the above initial conditions, which are simultaneously linked on the first ends of the three channels. From this figure we can see that the soliton in Pang's model can still move along the three channels at a constant speed and amplitude without dispersion. So Pang's soliton is thermally stable at $300 \mathrm{~K}$. In figure 41 we also show the results of soliton motion at a long time period of $120 \mathrm{Ps}$ and large spacings of 1000 sites (i.e., 333 amino acid residues are contained in each channel) at $300 \mathrm{~K}$ for the $\alpha$-helix protein molecules, when the above initial conditions are simultaneously linked on the first ends of the three channels. We see from this figure that the solitons are undisturbed in such conditions, and really move over a long time period and through large spacings along the protein molecular chains while retaining their amplitudes and velocities at bio-temperatures. In figure 42 we plot the collision behaviors of the solitons with clock shapes, set up from opposite ends of the channels in the $\alpha$-helix protein molecules, when the above initial conditions are simultaneously linked on the opposite ends of the three channels. From this figure we see clearly that the initial two solitons with clock shapes, separating 100 amino acid spacings in each channel, collide with each other at about 16ps. After the collision, the two solitons in each channel go through each other still retaining their clock shapes and propagating toward and separately along the three chains.

These results show clearly that although there are large lattice fluctuations in the protein molecules due to the influence of temperature, the nonlinear interaction between the amino acids and excitons is still able to stabilize the soliton, therefore Pang's soliton is very robust against thermal perturbation of the environment. Then, the lifetime of Pang's soliton is, at least, 120Ps. This means that Pang's soliton can play an important role in biological processes. 

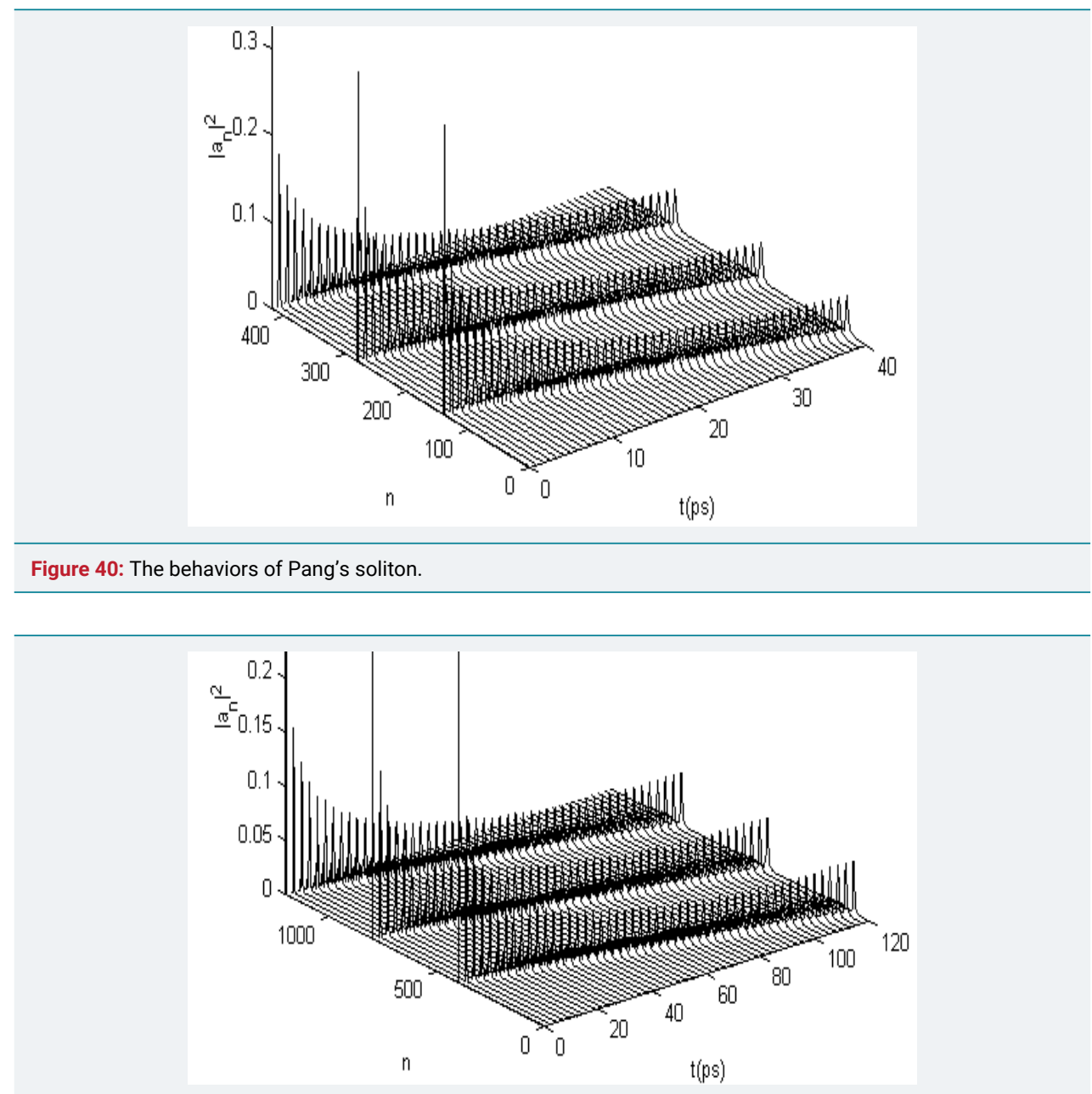

Figure 41: The state of the soliton in long-time at biological temperature of $300 \mathrm{~K}$. motion at $300 \mathrm{~K}$.

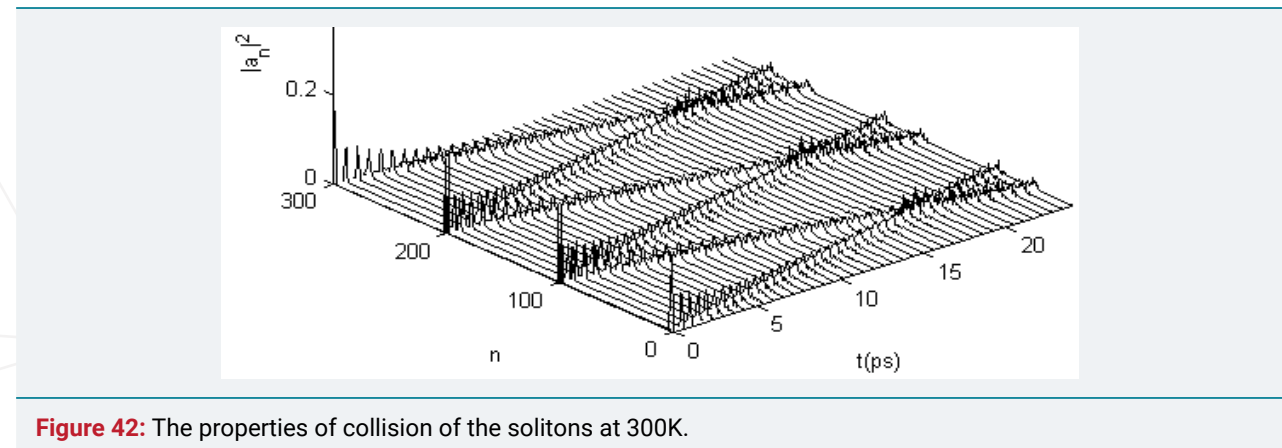

What does this mean? As mentioned above, the characteristic unit of time for the theories is $\tau_{0}=r_{0} / v_{0}=(M / W)^{1 / 2} \approx 0.98 \times 10^{-13} s$, and $L / r_{0}=100, \tau / \tau_{0}>500$ is a reasonable criterion for the soliton to be a possible mechanism for energy transport in proteins, where $\tau$ is the lifetime of the soliton. $\tau=300 p$ means $\tau / \tau_{0}>1000$. Thus, Pang's soliton is very thermally stable at $300 \mathrm{~K}$, i.e., Pang's oliton has a long enough lifetime enabling it to play an important role in biological processes.

We studied also the changes of states of the solitons with increasing temperature. In figure 43 we exhibit the transport properties of Pang's soliton in the $\alpha$-helix proteins at different temperatures of $295 \mathrm{~K}, 305 \mathrm{~K}, 310 \mathrm{~K}, 315 \mathrm{~K}, 320 \mathrm{~K}$ and $325 \mathrm{~K}$, respectively. These figures show that the amplitudes of the soliton decrease with increasing temperature, and it begins to disperse at 320K. This means that Pang's soliton must expend a part of itself energy to retain and suppress the increasing destructive effect of thermal 
perturbation arising from the rise in temperature of the system, thus its amplitude or energy does decrease. Thus we estimated that the critical temperature of Pang's soliton is about $320 \mathrm{~K}$ in this case. At the same time, we found that the transported velocity of Pang's soliton also decreases with the increase in temperature of the system

In table 2 we give concrete data of these velocities of Pang's soliton at different temperatures. Otherwise, the temperature-dependence of velocity of Pang's soliton is shown in figure 44 . Here, the decrease in soliton velocity with increasing temperature is also obvious. Evidently, this is due to the enhancement of disordered thermal motion of the medium resulting from the rise in temperature, which increases the resistance of motion of the soliton. Thus, the velocity of Pang's soliton necessarily decreases.

The above results of our investigation manifest clearly that the nonlinear interaction between the amino acids and excitons is still able to stabilize the soliton, although it undergoes destructive influences due to increases of thermal perturbation. Therefore, the soliton is very robust against the influence of thermal perturbation of the environment.

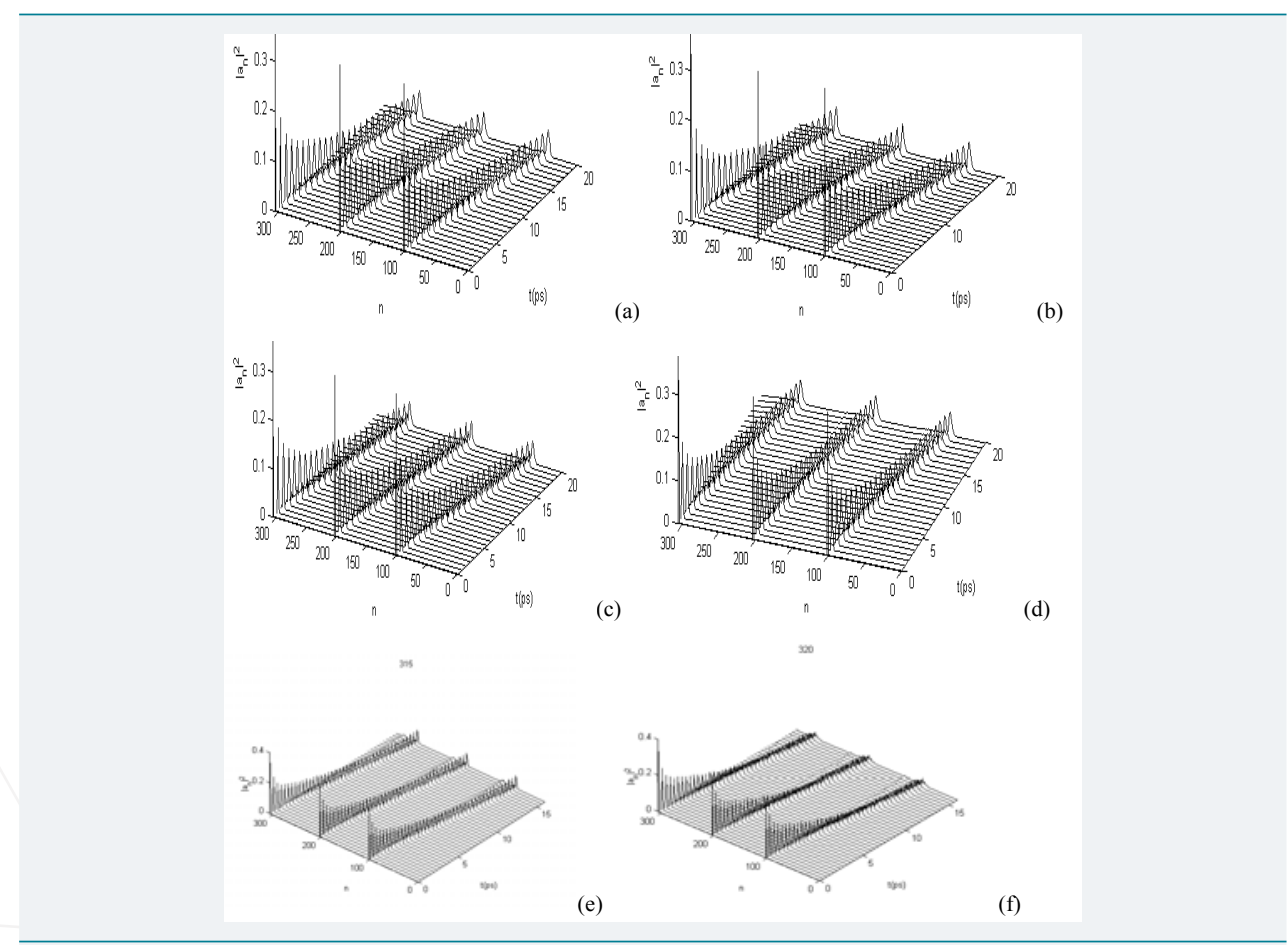

Figure 43: The changes of state of the solitons with increasing temperatures of the $\alpha$-helix protein molecules at temperatures of $295 \mathrm{~K}(\mathrm{a}), 305 \mathrm{~K}(\mathrm{~b}), 310 \mathrm{~K}(\mathrm{c}) 315 \mathrm{~K}(\mathrm{~d}), 320 \mathrm{~K}(\mathrm{e})$ and $325 \mathrm{~K}(\mathrm{f})$, respectively.

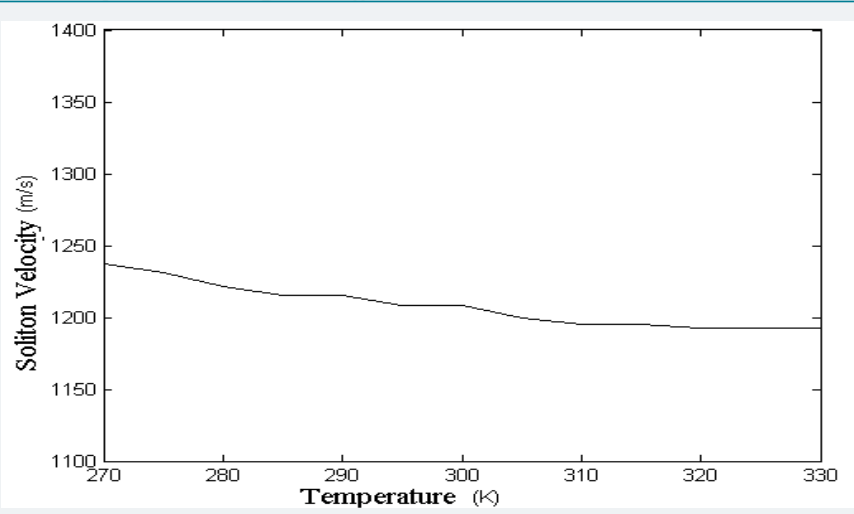

Figure 44: The temperature-dependence of velocity of the soliton excited in the -helix protein molecules. 
Table 2: The values of velocity of the solitons in the a-helix protein molecules at different temperatures.

\begin{tabular}{|l|l|l|l|l|l|l|l|l|l|l|l|l|}
\hline Temperature (K) & 270 & 275 & 280 & 285 & 290 & 295 & 300 & 305 & 310 & 315 & 320 & 325 \\
\hline
\end{tabular}

\begin{tabular}{|l|l|l|l|l|l|l|l|l|l|l|l|l|}
\hline velocity $(\mathrm{m} / \mathrm{s})$ & 1237 & 1230 & 1221 & 1215 & 1215 & 1208 & 1208 & 1199 & 1194 & 1194 & 1192 & 1191
\end{tabular}

The simultaneous effects of structure nonuniformity, damping and temperature of protein molecules on the energy transport

The results in single chain protein in Pang's model: However, the influence of structure nonuniformity or disorder in the protein molecules on the Pang's soliton has not been considered in the above calculations. In practice, the structure nonuniformity arising from the disorder in the mass sequence of amino acid molecules, side groups and imported impurities is always existent in the proteins. Therefore, it is quite necessary to further study the influence of structure nonuniformity on Pang's soliton in the region of 300-310K using the above method [114-125]. The behavior of Pang's soliton is shown in figure 45, when the disorder of the mass sequence is in the region of $\quad 0 . \bar{M}<M_{k}<2 \bar{M}$, where $\Delta J= \pm 5 \% \bar{J}, \Delta\left(\chi_{1}+\chi_{2}\right)= \pm 5 \% \overline{\left(\chi_{1}+\chi_{2}\right)}, \quad \Delta w= \pm 10 \% \overline{\mathrm{w}}$ , $\Delta \varepsilon_{0}=\varepsilon\left|\beta_{n}\right|$ and $\varepsilon=0.4 \mathrm{meV},|\beta| \leq 0.5$, for $\mathrm{T}=300 \mathrm{~K}, \mathrm{~T}=310 \mathrm{~K}, \mathrm{~T}=315 \mathrm{~K}$ and $\mathrm{T}=320 \mathrm{~K}$, respectively. These figures show clearly that Pang's soliton is still thermally stable at $\mathrm{T}<320 \mathrm{~K}$, but begins to disperse at $\mathrm{T}=320 \mathrm{~K}$. Thus, the critical temperature of Pang's soliton is not more than $320 \mathrm{~K}$ when structure nonuniformity exists. Therefore, we can conclude that Pang's soliton is very robust against thermal perturbation and structure nonuniformity among protein molecules at biological temperatures.

\section{The results in $\alpha$-helix protein molecules with three channels in Pang's model}

At the same time, we continuously studied the influence of structure nonuniformity of proteins on Pang's soliton at the biological temperature of $300 \mathrm{~K}$ in $\alpha$-helix proteins using the same method mentioned above. In figure 46, we plot the states of Pang's soliton at $\mathrm{T}=300 \mathrm{~K}$, while the disorder of the mass sequence is in the region of 0.67 $<\alpha_{k}<2$, and $\Delta\left(\chi_{1}+\chi_{2}\right)= \pm 4 \% \overline{\left(\chi_{1}+\chi_{2}\right)}, \Delta J= \pm 2 \% \bar{J}, \Delta w= \pm 4 \% \overline{\mathrm{W}}, \Delta \varepsilon_{0}=\varepsilon\left|\beta_{n}\right|$, $\varepsilon=0.5 \mathrm{meV},\left|\beta_{\mathrm{n}}\right| \leq 0.5$ are existed. From these figures we see clearly that Pang's soliton is undisturbed and still thermally stable at $300 \mathrm{~K}$ when these structure nonuniformities occur in the proteins. Therefore, we can conclude that Pang's soliton is robust against thermal perturbation and structure nonuniformity of the $\alpha$-helix protein molecules at biological temperatures. Thus, the soliton in Pang's model [113-125] is a exact carrier for bio-energy transport in the $\alpha$-helix protein molecules with three channels.

In figure 47 we exhibit the transport properties of the soliton in the $\alpha$-helix proteins at different temperatures of $300 \mathrm{~K}, 310 \mathrm{~K}, 315 \mathrm{~K}$ and $320 \mathrm{~K}$, respectively, when the fluctuations of six parameters of structure are $0.67 \bar{M}<M_{k}<2 \bar{M}, \Delta\left(\chi_{1}+\chi_{2}\right)= \pm 1 \%($ $\left.\bar{\chi}_{1}+\bar{\chi}_{2}\right), \Delta \mathrm{J}= \pm 0.7 \% \bar{J}, \Delta \mathrm{W}= \pm 7 \% \overline{\mathrm{W}}, \Delta \mathrm{L}= \pm 0.8 \% \bar{L}$ and $\Delta \varepsilon_{0}=\varepsilon\left|\beta_{n}\right|, \varepsilon=0.4 \mathrm{meV},\left|\beta_{\mathrm{n}}\right|$
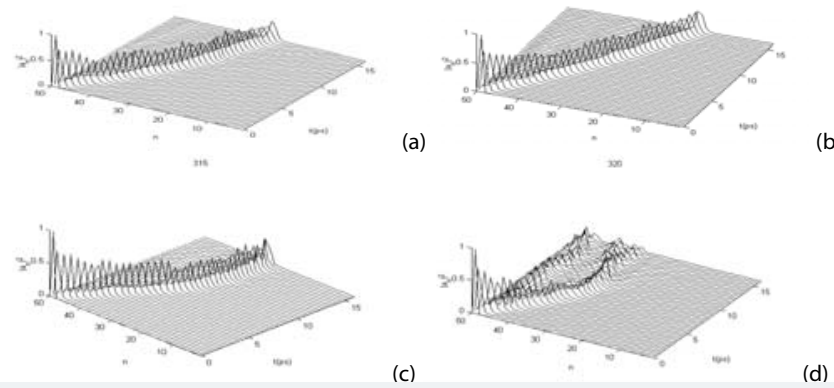

Figure 45: The state of Pang's soliton under the influence of disorder at $0.67 \bar{M}<M_{25}<2 \bar{M}, \Delta J= \pm 5 \% \bar{J}$ $\Delta\left(\chi_{1}+\chi_{2}\right)= \pm 5 \% \overline{\left(\chi_{1}+\chi_{2}\right)}, \quad \Delta w= \pm 10 \% \overline{\mathrm{W}}, \quad \Delta \varepsilon_{0}=\varepsilon\left|\beta_{n}\right|, \varepsilon=0.41 \mathrm{meV}, \quad\left|\beta_{n}\right|<1, \quad$ for $\mathrm{T}=300 \mathrm{~K}(\mathrm{a})$, $\mathrm{T}=310 \mathrm{~K}(\mathrm{~b}), \mathrm{T}=315 \mathrm{~K}(\mathrm{c})$, and $\mathrm{T}=320 \mathrm{~K}(\mathrm{~d})$. 


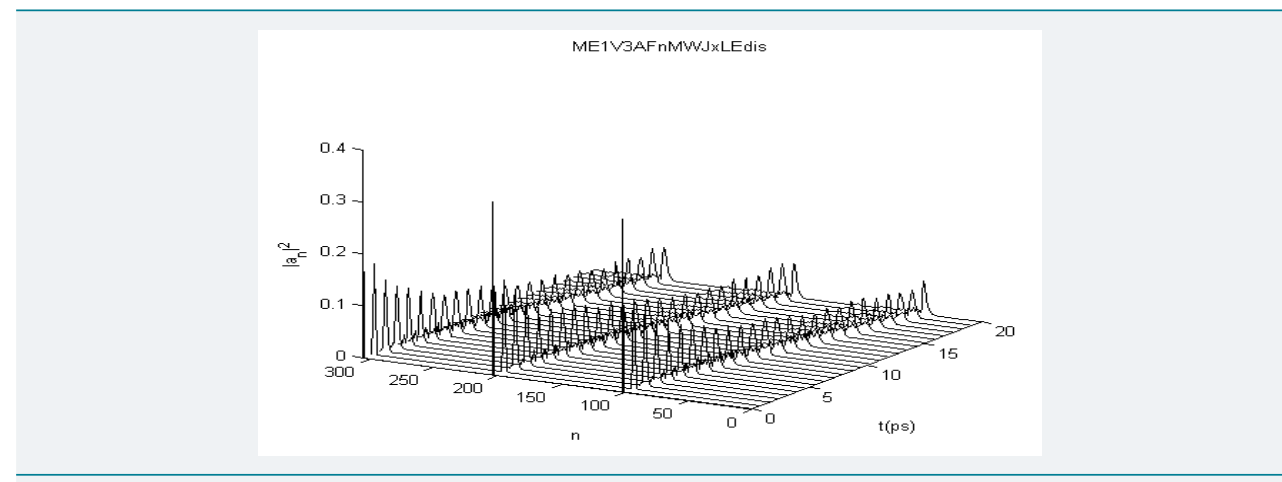

Figure 46: The states of Pang's soliton under the influences of the structure nonuniformities of $0.67 \bar{M}<M_{k}<2 \bar{M}$, $\Delta J= \pm 2 \% \bar{J}, \Delta\left(\chi_{1}+\chi_{2}\right)= \pm 4 \% \overline{\left(\chi_{1}+\chi_{2}\right)}, \Delta w= \pm 4 \% \bar{w}, \Delta \varepsilon_{0}=\varepsilon\left|\beta_{n}\right|, \varepsilon=0.5 \mathrm{meV},\left|\beta_{n}\right| \leq 0.5$ at $300 \mathrm{~K}$ in the $\alpha$-helix proteins.

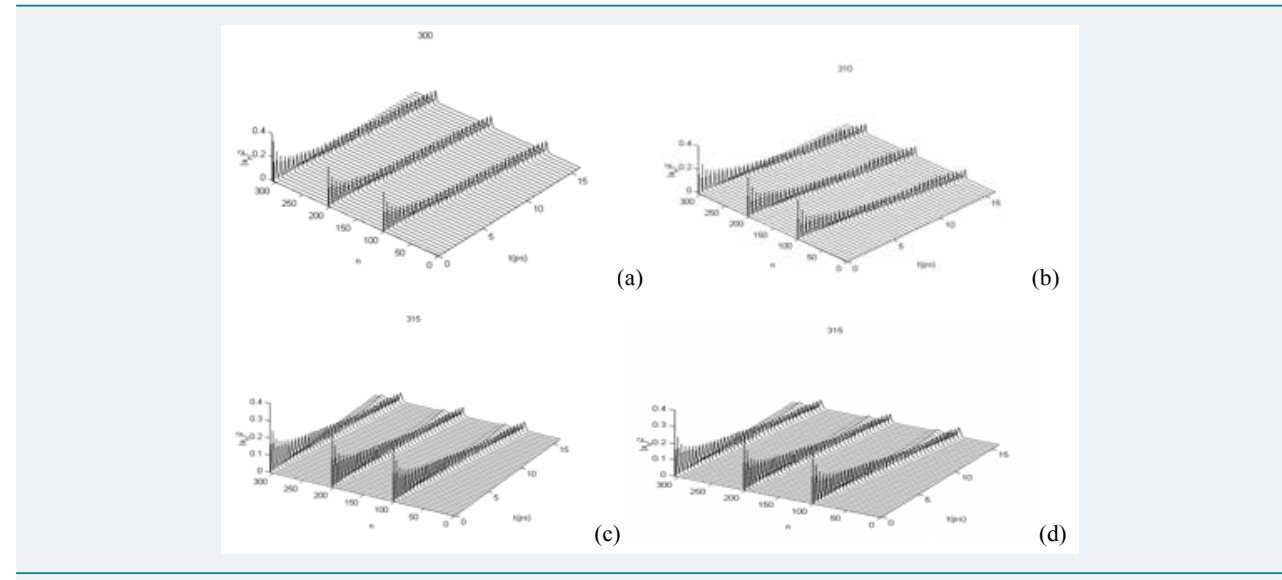

Figure 47: When the fluctuations_of structure parameters are $0.67 \bar{M}<M_{k}<2 \bar{M}, \Delta \mathrm{J}= \pm 0.7 \% \bar{J}, \Delta\left(\chi_{1}+\chi_{2}\right)= \pm 1 \%($ $\left.\bar{\chi}_{1}+\bar{\chi}_{2}\right), \Delta \mathrm{W}= \pm 7 \% \overline{\mathrm{W}}, \Delta \mathrm{L}= \pm 0.8 \% \bar{L}$ and $\Delta \varepsilon_{0}=\varepsilon\left|\beta_{n}\right|, \varepsilon=0.4 \mathrm{meV},\left|\beta_{n}\right| \leq 0.5$ the peculiarities of the soliton atT=300K(a), $310 \mathrm{~K}(\mathrm{~b}), 315 \mathrm{~K}(\mathrm{c})$ and $320 \mathrm{~K}$,respectively.

$\leq 0.5$. This figure shows that the amplitudes of Pang's soliton decrease with increasing temperature, and it begins to disperse at $315 \mathrm{~K}$. This manifests that the soliton must expend itself a part of energy to suppress the increase of destructive effect resulting from the thermal perturbation due to the lift of temperature of the systems, thus its amplitude or energy does depress. We drew from this result that the critical temperature of Pang's soliton is about $315 \mathrm{~K}$ in this condition. Obviously, decreases of the velocity of Pang's soliton with increasing temperature are due to the enhancement of disorder thermal motion of medium, resulting from the lift of temperature, which increases the resistance of motion of the soliton. However, the Pabng's soliton is still robust against the structure nonuniformity of the protein molecules and thermal perturbation and damping of the medium, thus its critical temperature can reach still $315 \mathrm{~K}$ in this case. Thus we conclude that Pang's soliton can really play an important role in the bioenergy transport, Pang's theory of bio-energy transport is really appropriate to the $\alpha$ -helix proteins, which is consistent with analytic results [105-107].

\section{CONCLUSION}

In this review article we must look at whether the theories including Davydov's and Pang's models are appropriate to the biological proteins in the living systems. This is due to the fact that these theoretical models were built based on a periodic, uniform and infinite proteins molecules, which are an ideal protein model and different from the biological proteins molecules in living systems. In this investigation the influences of structure nonuniformity and disorder, side groups and imported impurities of protein chains as well as the thermal perturbation and damping of medium arising 
from the biological temperature of the system on the bio-energy transport were studied carefully by many scientists using different techniques and methods in past thirty years.

In this article we review systematically and completely the correctness of the theory of bio-energy transport in the biological proteins including Davydov's and Pang's models. These results provided in the review were obtained by numerical simulation techniques using various methods, for example, the average Hamiltonian way of thermal perturbation, fourth-order Runge-Kutta method, Monte Carlo method, quantum perturbed way and thermodynamic and statistical method, in which no approximations are used. However, we mainly inspect and check the validity and availability of Davydov model and Pang's model in single chain protein molecules and $\alpha$-helical protein molecules with three channels, respectively. Concretely speaking, we studied the four problems, i.e., the properties of bio-energy transport in uniform protein molecules; the influence of structure nonuniformity on the bio-energy transport; the effects of temperature of systems on the bio-energy transport and the simultaneous effects of structure nonuniformity, damping and thermal perturbation of proteins on the bio-energy transport in a single chains and $\alpha$-helical molecules. We conclude from these investigations that the Davydov soliton is not stable under the influence of small structure noniniformity and extremely unstable at $300 \mathrm{~K}$ under influences of larger structure disorders, but Pang's soliton is stable at physiologic temperature $300 \mathrm{~K}$ and very robust against these structure nonuniformities and the influences of side groups, imported impurities and damping of medium. Accurately speaking, If the effects of thermal perturbation of medium on the soliton in nonuniform proteins is considered, the Pang's soliton can transport over a larger spacing of 400 amino acids and has a longer lifetime of 300PS, and begins only to disperse at a higher temperature of $325 \mathrm{~K}$ and large structure nonuniformity in both a single chain and three chain protein molecules,. its critical temperature can reach $320 \mathrm{~K}$. in this case. Concretely speaking, for the nonuniform fluctuations of $0.67<\alpha_{\mathrm{K}}<2, \Delta \mathrm{W}= \pm 8 \% \overline{\mathrm{W}}, \Delta \mathrm{J}= \pm 1 \% \bar{J}$ $\Delta\left(\chi_{1}+\chi_{2}\right)= \pm 3 \%\left(\bar{\chi}_{1}+\bar{\chi}_{2}\right)$ and $\Delta \mathrm{L}= \pm 1 \% L$ and $\Delta \varepsilon_{0}=\varepsilon\left|\beta_{\mathrm{n}}\right|, \varepsilon=0.1 \mathrm{meV},\left|\beta_{\mathrm{n}}\right|<0.5$, Pang's soliton is still stable in the $\alpha$-helical protein molecules.. When the effects of structure nonuniformity and temperature are considered simultaneously, it has still high thermal stability and can transport also along the protein molecular chains retaining its amplitude, energy and velocity. However, the soliton begins to disperse in the larger fluctuations, for example, $0.67 \bar{M} \leq M_{k}<2 \bar{M}, \Delta \mathrm{W}= \pm 6 \% \overline{\mathrm{W}}, \Delta \mathrm{J}= \pm 1.3 \%$ $\bar{J}, \Delta\left(\chi_{1}+\chi_{2}\right)= \pm 2 \%\left(\bar{\chi}_{1}+\bar{\chi}_{2}\right), \Delta \mathrm{L}= \pm 1.5 \% L$ and $\Delta \varepsilon_{0}=\varepsilon\left|\beta_{n}\right|, \quad \varepsilon=0.82 \mathrm{meV}$, $\left|\beta_{\mathrm{n}}\right| \leq 0.5$ at $\mathrm{T}=300 \mathrm{~K}$,or the temperatures higher than $315 \mathrm{~K}$ and the fluctuations of $0.67 \bar{M}<M_{k}<2 \bar{M}, \Delta\left(\chi_{1}+\chi_{2}\right)= \pm 1 \%\left(\bar{\chi}_{1}+\bar{\chi}_{2}\right), \Delta \mathrm{J}= \pm 0.7 \% \bar{J}, \Delta \mathrm{W}= \pm 7 \% \overline{\mathrm{W}}$, $\Delta \mathrm{L}= \pm 0.8 \% L$ and $\Delta \varepsilon_{0}=\varepsilon\left|\beta_{n}\right|, \varepsilon=0.4 \mathrm{meV},\left|\beta_{\mathrm{n}}\right| \leq 0.5$. Its critical temperature is $315 \mathrm{~K}$ in this condition. These results are similar with analytic conclusions [105-107]. Thus, we can conclude that the soliton in Pang's model is exactly a carrier of the bio-energy transport, Pang's theory is appropriate to $\alpha$-helical protein molecules.

\section{Acknowledgement}

* Author would like to acknowledge the National Basic Research Program of China (“973” program) for the financial support (Grant No. 212011CB503701)

\section{Refrences}

1. Pang Xiao-feng, Biophysics, The press of Univ. of Electronic Sci. Techno of China, Chengdu. 2007.

2. Szent-Gyorgy A. Towards a New Biochemistry. Science. 1941; 93: 609-611. Ref.: https://goo.gl/sWcamx

3. Bakhshi AK, Otto P, Ladik J, Seel M. Chem Phys. 1986; 20: 687.

4. Schulz GE, Schirmar RH, Principles of protein molecules. Springer. 1979.

5. Davydov AS. The theory of contraction of proteins under their excitation. J Theor Biol. 1973; 38: 559- 
569. Ref.: https://goo.gl/eD18C2

6. Davydov AS. Solitons and energy transfer along protein molecules. J Theor Biol. 1977; 66: 379-387. Ref.: https://goo.gl/d3gYhb

7. Davydov AS. Solitons in Molecular Systems. Phys Scr. 1979; 20: 387. Ref.: https://goo.gl/ncUh61

8. Hyman JM, McLaughlin DW, Scott AC. On Davydov's alpha-helix solitons. Physica 1981; 3: 23-44. Ref.: https://goo.gl/AqFHcb

9. Davydov AS. Sov Phys USP. 1982; 25: 898.

10. Davydov AS. Biology and quantum mechanics. Pergamon. 1982.

11. Davydov AS. The solitons in molecular systems. Reidel. 1985.

12. Davydov AS, Kislukha NI. Solitons in One-Dimensional Molecular Chains. Phys Stat Sol. 1973; 59: 465. Ref.: https://goo.gl/S79fp5

13. Davydov AS, Kislukha NI, Phys Stat Sol. 1977; $75: 735$.

14. Brizhik LS, Davydov AS. Soliton excitations in one-dimensional molecular systems. Phys Stat Sol. 1983; 115: 615-630. Ref.: https://goo.gl/vbQUNf

15. Scott AC. Dynamics of Davydov solitons. Phys Rev A. 1982; 26: 578. Ref.: https://goo.gl/EjDG4d

16. Scott AC. Dynamics of Davydov solitons. Phys Rev A. 1983; 27: 2767. Ref.: https://goo.gl/avWRcS

17. Scott AC. The Vibrational Structure of Davydov Solitons. Phys Scr. 1982; 25: 651. Ref.: https://goo. $\mathrm{gl} / 27 \mathrm{Yzh}$

18. Scott AC. Launching a Davydov Soliton: I. Soliton Analysis. Phys Scr. 1984; 29: 279. Ref.: https://goo. $\mathrm{gl} / \mathrm{d} 8$ tyZp

19. Scott AC. Davydov's soliton. Phys Rep. 1992; 217: 1-67. Ref.: https://goo.gl/UF4wXJ

20. Scott AC. Physica. 1990; 51: 333.

21. Brown DW, West BJ, Lindenberg K. Phys Rev A. 1986; 33: 4104.

22. Brown DW, West BJ, Lindenberg K. Davydov solitons: New results at variance with standard derivations. Phys Rev A Gen Phys. 1986; 33: 4110-4120. Ref.: https://goo.gl/U36Cyg

23. Brown DW, Lindenberg K, West BJ. Phys Rev B. 1987; 35: 6169.

24. Brown DW, Lindenberg K, West BJ. Phys Rev B. 1988; 37: 2946.

25. Brown DW, Lindenberg K, West BJ. Phys Rev Lett. 1986; 57: 234.

26. Brown DW. Phys Rev A. 1988; 37: 5010.

27. Brown DW. Ivic Z. Phys Rev B. 1989; 40: 9876.

28. Ivic Z, Brown DW. Phys Rev Lett. 1989; 63: 426.

29. Skrinjar MJ, Kapor DW, Stojanovic SD. Phys RevA. 1988; 38: 6402.

30. Skrinjar MJ, Kapor DW, Stojanovic SD. Phys Rev B. 1989; 40: 1984.

31. Skrinjar MJ, Kapor DW, Stojanovic SD. Phys Lett A. 1988; 133: 489.

32. Skrinjar MJ, Kapor DW, Stojanovic SD. Phys Scr. 1988; 39. 658.

33. Pang Xiao-feng. Chin J Biochem Biophys. 1986; $18: 1$.

34. Pang Xiao-feng. Chin J Atom Mol Phys. 1986; 6: 275.

35. Pang Xiao-feng. Chin J Appl Math. 1986; 10: 278.

36. Christiansen PL, Scott AC. Davydov's soliton revisited: Self-trapping of vibrational energy. Plenum Press. 1990. Ref.: https://goo.gl/vp52Y6

37. Davydov AS, Zh Eksp Teor Fiz. 1980; 78: 789.

38. Davydov AS. The lifetime of molecular (Davydov) solitons. J Biol Phys. 1991; 18: 111-125. Ref.: https://goo.gl/61DRMB 
39. Cruzeiro L, Halding J, Christiansen PL, Skovgard O, Scott AC. Phys Rev A. 1985; 37: 703.

40. Cruzeiro L. Proteins multi-funnel energy landscape and misfolding diseases. J Phys Org Chem. 2008; 21, 549-554. Ref.: https://goo.gl/jGL7mj

41. Cruzeiro L. Influence of the sign of the coupling on the temperature dependence of optical properties of one-dimensional exciton models. J Phys B: At Mol Opt Phys. 2008; 41: 195401. Ref.: https://goo. gl/PfdHnN

42. Cruzeiro L. The Davydov/Scott Model for Energy Storage and Transport in Proteinsm. J Bio Physics. 2009; 35: 43-55. Ref.: https://goo.gl/tn9DNP

43. Cruzeiro L. J Chem Phys. 2005; 123: 4909.

44. Cruzeiro L. J Phys: Condens Matter. 2005; 17: 7833-7844.

45. Cruzeiro-Hansson L. Phys Rev A. 1992; 45: 4111.

46. Cruzeiro-Hansson L. Physica D. 1993; 68: 65.

47. Cruzeiro-Hansson L. Two Reasons Why the Davydov Soliton May Be Thermally Stable After All. Phys Rev Lett. 1994; 73: 2927. Ref.: https://goo.gl/dBb6L8

48. Cruzeio-Hansson L, Kenker VM, Scott AC. Phys Lett A. 1994; 190: 59.

49. Cruzeiro-Hansson L, Takeno S. Davydov Model: The Quantum, Mixed Quantum-Classical and Full Classical Systems. Phys Rev E. 1997; 56: 894-906. Ref.: https://goo.gl/wA69g5

50. Forner W. Quantum and disorder effects in Davydov soliton theory. Phys Rev A. 1991; 44: 2694-2708. Ref.: https://goo.gl/hxgPWm

51. Forner W. Physica D. 1993; 68: 68.

52. Forner W. J Comput Chem. 1992; 13: 275.

53. Forner W. Davydov soliton dynamics: temperature effects. J Phys Condensed Matter. 1991; 3: 1915. Ref.: https://goo.gl/oA52P6

54. Forner W. J Phys Condensed Matter. 1992; 4: 4333.

55. Forner W. Quantum and temperature effects on Davydov soliton dynamics. IV. Lattice with a thermal phonon distribution. J Phys Condensed Matter. 1993; 5: 823. Ref.: https://goo.gl/xU7y5Z

56. Forner W. J Phys Condensed Matter. 1993; 5: 883.

57. Forner W. Launching a Davydov Soliton: II. Numerical Studies. J Phys Condensed Matter. 1993; 5: 3883. Ref.: https://goo.gl/NrhAug

58. Forner W. Quantum and temperature effects on Davydov soliton dynamics. V. Numerical estimate of the errors introduced by the ID1> ansatz. J Phys Condensed Matter. 1993; 5: 3897. Ref.: https://goo. $\mathrm{gl} / \mathrm{xEH} \mathrm{Hco6}$

59. Motschman H, Forner W, Ladik J. J Phys Condensed Matter. 1989; 1: 5083.

60. Forner W. J Phys Condensed Matter. 1994; 6: 9089-9151.

61. Forner W. J Mol Model. 1996; 2: 70-135.

62. Lomdahl PS, Kerr WC. Do Davydov Solitons Exist at 300 K?. Phys Rev Lett. 1985; 55: 1235. Ref.: https://goo.gl/nZVG4M

63. Kerr WC, Lomdahl PS. Phys Rev B. 1989; 35: 3629.

64. Wang X, Brown DW, Lindenberg K. Phys Rev Lett. 1989; 62: 1792.

65. Wang X, Brown DW, Lindenberg K. Phys Rev A. 1988; 37: 3357.

66. Cottingham JP, Schweitzer JW. Phys Rev Lett. 1989; 62: 1792.

67. Schweitzer JW. Phys Rev A. 1992; 45: 8914.

68. Hyman JM, Mclaughlin DW, Scott AC. Physica D. 1981; 3: 23.

69. Lawrence AF, McDaniel JC, Chang DB, Pierce BM, Brirge RR. Phys Rev A. 1986; 33: 1188. 
70. Mechtly B, Shaw PB. Evolution of a molecular exciton on a Davydov lattice at $T=0$. Phys Rev B. 1988; 38: 3075. Ref.: https://goo.gl/h6MAfm

71. Macneil L, Scott AC. Launching a Davydov Soliton: II. Numerical Studies. Phys Scr. 1984; 29: 284. Ref.: https://goo.gl/uGPGxS

72. Bolterauer H, Opper M, Z Phys B. 1991; 82: 95.

73. Eibeck JC, Lomdahl PS, Scott AC. Phys Rev B. 1984; 30: 4703.

74. Forner W. J Phys Condensed Matter. 1991; 3: 3235.

75. Takeno S. Vibron Solitons in One-Dimensional Molecular Crystals. Prog Theor Phys. 1984; 71: 395398. Ref.: https://goo.gl/aHoKxr

76. Takeno S. Vibron Solitons and Coherent Polarization in an Exactly Tractable Oscillator-Lattice System: Applications to Solitons in a Helical Proteins and Fröhlich's Idea of Biological Activity. Prog Theor Phys. 1985; 73: 853-873. Ref.: https://goo.gl/AikZa9

77. Takeno S. J Phys Soc. 1991; 59: 3127.

78. Pang Xiao-feng. The properties of the collective excitation in the organic protein molecular system. J Phys Condensed Matter. 1990; 2: 9541. Ref.: https://goo.gl/ntwSW6

79. Pang Xiao-feng. Phys Rev E. 1994; 49: 4747.

80. Pang Xiao-feng. European Phys J B. 1999; 10: 415.

81. Pang Xiao-feng. Chin Phys Lett. 1993; 10: 381.

82. Pang Xiao-feng. Quantum-Mechanical Method for the Soliton Transported Bio-energy in Protein. Chin Phys Lett. 1993; 10: 437. Ref.: https://goo.gl/Msk85t

83. Pang Xiao-feng. Stability of the Soliton Excited in Protein in the Biologic Temperature Range. Chin Phys Lett. 1993; 10: 573-576. Ref.: https://goo.gl/2HTSxw

84. Pang Xiao-feng. Chin Science Bulletin. 1993; 38: 1572.

85. Pang Xiao-feng. Chin Science Bulletin. 1993; 38: 1665.

86. Pang Xiao-feng. Chin J Biophys. 1993; 9: 637.

87. Pang Xiao-feng. Chin J Biophys. 1994; 10: 133.

88. Pang Xiao-feng. Chin J Bio-chem Biophys. 1986; 18: 1-8.

89. Pang Xiao-feng. Acta Math Sci. 1993; 13: 437.

90. Pang Xiao-feng. Acta Math Sci. 1996; 16: 1.

91. Pang Xiao-feng. Acta Phys Sinica. 1993; 42: 1856.

92. Pang Xiao-feng. Acta Phys Sinica. 1997; 46: 625.

93. Pang Xiao-feng. Chin J Infrared Millimeter Waves. 1993; 12: 377.

94. Pang Xiao-feng. Chin J Infrared Millimeter Waves. 1997; 16: 64.

95. Pang Xiao-feng. Chin J Infrared Millimeter Waves. 1997; 16: 301.

96. Pang Xiao-feng. Chin J Atom Mol Phys. 1987; 5: 383.

97. Pang Xiao-feng. Chin J Atom Mol Phys. 1995; 12: 411.

98. Pang Xiao-feng. Chin J Atom Mol Phys. 1996; 13: 70.

99. Pang Xiao-feng. Chin J Atom Mol Phys. 1997; 14: 232.

100. Pang Xiao-feng. The theory for non linear quantum mechanics, Chinese Chongqing Press, Chongqing. Sci Res. 1994; 415-686. Ref.: https://goo.gl/sfkhix

101. Pang Xiao-feng. Acta Phys Slovaca. 1998; 47: 89.

102. Pang Xiao-feng. J Phys Condensed Matter. 2000; 12: 885.

103. Pang Xiao-feng. Chinese Physics. 2000; 9: 86. 
104. Pang Xiao-feng. Phys Rev E. 2000; 62: 6989.

105. Pang Xiao-feng. European Phys J B. 2001; 19: 297

106. Pang Xiao-feng. Commun Theor Phys. 2001; 35: 323.

107. Pang Xiao-feng. Commun Theor Phys. 2002; 36: 178.

108. Pang Xiao-feng. J Int Inf Mill Waves. 2001; 22: 291.

109. Pang Xiao-feng. J Phys Chem Solids. 2001; 62: 793.

110. Pang Xiao-feng. Chin J BioMed Engineering. 1999; 8: 39.

111. Pang Xiao-feng. Chin J BioMed Engineering. 2001; 10: 613.

112. Pang Xiao-feng, Feng Yuan-ping. Quantum mechanics in nonlinear systems. World Science Publishing Co. 2005; 471-551.

113. Pang Xiao-feng, Zhang Huai-wu, Yu Jia-feng, Feng Yuan-ping. States and properties of the soliton transported bio-energy in nonuniform protein molecules at physiological temperature. Phys Lett $A$. 2005; 335: 408-416. Ref.: https://goo.gl/iv5LJX

114. Pang Xiao-feng, Luo Yui-hue. Commun Theor Phys. 2004; 41: 470.

115. Pang Xiao-feng, Luo Yui-hue. Commun Theor Phys. 2005; 43: 367.

116. Pang Xiao-feng, Yu Jia-feng, Luo Yu-hui. Commun Theor Physics. 2005; 43: 367-376.

117. Pang Xiao-feng, Zhang Huai-wu. J Physics Chemistry Solids. 2005; 66: 963- 972.

118. Pang Xiao-feng , Zhang Huai-Wu, Yu Jia-feng, Luo Yu-hui. Int J Modern Physics B. 2005; 19: 46774699.

119. Pang Xiao-feng, Zhang Huai-wu, Yu Jia-feng. J Phys Condensed Matter. 2006; 18: 613-627.

120. Pang Xiao-feng, Zhang Huai-Wu,Yu Jia-feng, Luo yu-hui. Int J Modern Physics B. 2006; 20: 3027.

121. Pang Xiao-feng, Yu Jia-feng, Lao Yu-hui. Inter J Mod Phys B. 2007; 21: 13-42.

122. Pang Xiao-feng, Liu Mei-jie. Commun Theory Physics. 2007; 48: 369-376.

123. Pang Xiao-feng. Influence of structure disorders and temperatures of systems on the bio-energy transport in protein molecules (II). Frontiers Physics China. 2008; 3: 457-488. Ref.: https://goo.gl/VEDfjP

124. Pang Xiao-Feng, LIU Mei-Jie. Commun Theor Phys. 2009; 51: 170-180.

125. Pang Xiao-feng, Yu Jia-feng, Liu Mei-jie. Molecular Physics. 2010; 108: 1297-1315.

126. Fohlich H. Adv Electron Electron Phys. 1980; 53; 86.

127. Spatschek KH, Mertens FG. Nonlinear coherent structures in physics and Biology. Plenum Press. 1994.

128. Popp FA, Li KH, Gu Q. Recent advances in biophoton research and its application. World Scientific. $1993 ; 141$.

129. Mae Wan Ho, Popp FA, Warnke U. Bioelectrodynamics and Biocommunication. Would Scientific. $1994 ; 87$.

130. Pang Xiao-feng. soliton physics; Chinese Sichuan Science and Technology Press. Chengdu. 2000; P2-180.

131. Guo Bai-lin, Pang Xiao-feng. Solitons, Chinese Science Press. Beijing. 1987; P4-38. 340.

132. Bullough PK, Caudrey PJ. Sliton Spinger. 1982; P80-160.

133. Young E, Shaw PB, Whitfield G. Phys Rev B. 1979; 19: 1225.

134. Venzl G, Fischer SF. J Phys Chem. 1984; 81: 6090.

135. Nagle JF, Mille M, Morowitz HJ. Chem J Phys. 1980; 72: 3959.

136. Wanger M, Kongeter A. Chem J Phys. 1989; 91: 3036.

137. Eremko AA, Yu Gaididei B, Vakhnenko AA. Dissociation-Accompanied Raman Scattering by Davydov Solitons. Phys Stat Sol B. 1985; 127: 703-713. Ref.: https://goo.gl/dBchQX 
138. Careri GA, Gransanti, Ruple JA. Phys Rev A. 1998; 37: 2703.

139. Careri G, Gratton E, Shyamsunder E. Phys Rev A. 1998; 37: 4048.

140. Careri G, Buontempo U, Galluzzi F, Scott AC, Gratton Eet al. Spectroscopic evidence for Davydov-like solitons in acetanilide. Phys Rev B. 1984; 30: 4689. Ref.: https://goo.gl/CMF1ao

141. Careri G, Buonttempo U, Caeta F, Scott AC. Phys Rev Lett. 1983; 51: 304.

142. Scott AC. Physica D. 1990; 51: 333.

143. Scott AC. Phys Lett A. 1998; 86: 603.

144. MacNeil L, Scott AC. Phys Scr. 1984; 22: 842-879.

145. Kerr WC, Lomdahl PS. Quantum mechanical derivation of the equation of motion for Davydov equarions for muilti-quanta states, in Davydov's solitons revisited, eds. Christiansen PS, Scott AC. Plenum. 1990; 23-30.

146. Rupley LA, Nicholls A. Phys Rev Lett. 1990; 64: 1174-1177.

147. Stiefel J. Einfuhrung in die Numerische Mathematik Teubner Verlag. Stuttgart. 1965; 76-123.

148. Atkinson KE. An Introduction to Numerical Analysis. Wiley. 1987; 47-186.

149. Forner W. J Phys Condens Matter. 1993; 5: 805-823.

150. Forner W, Ladik J. influence of heat bath and disorder on Davydov solitons, in Davydov's Soliton Revisited: Self-Trapping of Vibrational Energy in Proteins, ed by Christiansen PL and Scott AC. Plenum. 1991; 267-284.

151. Forner W. Davydov soliton dynamics: two-quantum states and diagonal disorder. J Phys Condens Matter. 1991; 3: 3235. Ref.: https://goo.gl/NNPzJc

152. HofmannD, Forner W, Ladik J. J Phys Condensed Matter. 1990; 2: 4081.

153. Davydov AS. Sov Phys JETP. 1980; 51: 397-400.

154. Cruzeiro L, Halding J, Christiansen PL, Skovgaard G, Scott AS. Phys Rev A. 1988; 37: 880-887.

155. Kenkre VM, Raghavan S, Cruzeiro-Hansson L. Thermal Stability of Extended Nonlinear Structures Related to the Davydov Solito. Phys Rev B. 1994; 49: 9511-9522. Ref.: https://goo.gl/rkkyRY

156. Bolterauer H. Temperature effects on the Davydov soliton, in Self-trapping of vibrational energy, ed by Christiansen PL and Scott AC. Plenum Press. 1990; 309-323.

157. Lawrence AF, McDanied JC, Chang DB, Birge RR. Biophys J. 1987; 51: 785-793.

158. Lomdahl PS, Kerr WC. Davydov soliton at 300kelven: the final search, in Self-trapping of vibrational energy, ed by Christiansen PL and Scott AC. Plenum Press. 1990; 259-265. 